\title{
Outer-disk reddening and gas-phase metallicities: The CALIFA connection
}

\author{
R. A. Marino1,2, A. Gil de Paz ${ }^{3}$, S. F. Sánchez ${ }^{4}$, P. Sánchez-Blázquez ${ }^{5}$, N. Cardiel ${ }^{3,6}$, A. Castillo-Morales ${ }^{3}$, S. Pascual ${ }^{3}$, \\ J. Vílchez ${ }^{11}$, C. Kehrig ${ }^{11}$, M. Mollá7 ${ }^{7}$ J. Mendez-Abreu ${ }^{8}$, C. Catalán-Torrecilla ${ }^{3}$, E. Florido ${ }^{9,10}$, I. Perez ${ }^{9,10}$, \\ T. Ruiz-Lara ${ }^{9,10}$, S. Ellis ${ }^{12}$, A. R. López-Sánchez ${ }^{12,13}$, R. M. González Delgado ${ }^{11}$, A. de Lorenzo-Cáceres ${ }^{8}$, \\ R. García-Benito ${ }^{11}$, L. Galbany ${ }^{14,15}$, S. Zibetti ${ }^{16}$, C. Cortijo ${ }^{11}$, V. Kalinova ${ }^{17}$, D. Mast ${ }^{18}$, J. Iglesias-Páramo ${ }^{11,19}$, \\ P. Papaderos ${ }^{20,21}$, C. J. Walcher ${ }^{22}$, J. Bland-Hawthorn ${ }^{23}$, and the CALIFA Team ${ }^{19, \star}$
}

${ }^{1}$ CEI Campus Moncloa, UCM-UPM, Departamento de Astrofísica y CC. de la Atmósfera, Facultad de CC. Físicas, Universidad Complutense de Madrid, Avda. Complutense s/n, 28040 Madrid, Spain e-mail: ramarino@ucm.es

2 Department of Physics, Institute for Astronomy, ETH Zürich, 8093 Zürich, Switzerland

3 Departamento de Astrofísica y CC. de la Atmósfera, Facultad de CC. Físicas, Universidad Complutense de Madrid, Avda. Complutense s/n, 28040 Madrid, Spain

${ }^{4}$ Instituto de Astronomía, Universidad Nacional Autonóma de México, A.P. 70-264, 04510 México, D. F., Mexico

5 Departamento de Física Teórica, Universidad Autónoma de Madrid, 28049 Madrid, Spain

${ }^{6}$ Instituto de Física de Cantabria (CSIC -Universidad de Cantabria), Avenida de los Castros s/n, 39005 Santander, Spain

7 CIEMAT, Departamento de Investigación Básica, Avda. Complutense 40, 28040 Madrid, Spain

8 School of Physics and Astronomy, University of St Andrews, North Haugh, St. Andrews, KY16 9SS, UK (SUPA)

9 Instituto Universitario Carlos I de Física Teórica y Computacional, Universidad de Granada, 18071 Granada, Spain

10 Departamento de Física Teórica y del Cosmos, Facultad de Ciencias, Universidad de Granada, 18071 Granada, Spain

11 Instituto de Astrofísica de Andalucía (IAA/CSIC ), Glorieta de la Astronomía s/n Aptdo. 3004, 18080 Granada, Spain

12 Australian Astronomical Observatory, PO Box 915, North Ryde, NSW 1670, Australia

13 Department of Physics and Astronomy, Macquarie University, NSW 2109, Australia

14 Millennium Institute of Astrophysics MAS, Nuncio Monseñor Sótero Sanz 100, Providencia, 7500011 Santiago, Chile

15 Departamento de Astronomía, Universidad de Chile, Camino El Observatorio 1515, Las Condes, Santiago, Chile

16 INAF-Osservatorio Astrofisico di Arcetri - Largo Enrico Fermi, 550125 Firenze, Italy

17 Department of Physics 4-181 CCIS, University of Alberta, Edmonton AB T6G 2E1, Canada

18 Instituto de Cosmologia, Relatividade e Astrofísica, Centro Brasileiro de Pesquisas Físicas, CEP 22290-180 Rio de Janeiro, Brazil

19 Estación Experimental de Zonas Aridas (CSIC), Carretera de Sacramento s/n, La Cañada, 04120 Almería, Spain

20 Instituto de Astrofísica e Ciências do Espaço, Universidade do Porto, 4150-762 Porto, Portugal

21 Centro de Astrofísica da Universidade do Porto, Rua das Estrelas, 4150-762 Porto, Portugal

22 Leibniz-Institut für Astrophysik Potsdam (AIP), An der Sternwarte 16, 14482 Potsdam, Germany

23 Sydney Institute for Astronomy, School of Physics A28, University of Sydney, NSW2006, Australia

Received 17 July 2015 / Accepted 10 September 2015

\section{ABSTRACT}

\begin{abstract}
We study, for the first time in a statistically significant and well-defined sample, the relation between the outer-disk ionized-gas metallicity gradients and the presence of breaks in the surface brightness profiles of disk galaxies. Sloan Digital Sky Survey (SDSS) $g^{\prime}$ - and $r^{\prime}$-band surface brightness, $\left(g^{\prime}-r^{\prime}\right)$ color, and ionized-gas oxygen abundance profiles for 324 galaxies within the Calar Alto Legacy Integral Field Area (CALIFA) survey are used for this purpose. We perform a detailed light-profile classification, finding that $84 \%$ of our disks show down- or up-bending profiles (Type II and Type III, respectively), while the remaining $16 \%$ are well fitted by one single exponential (Type I). The analysis of the color gradients at both sides of this break shows a U-shaped profile for most Type II galaxies with an average minimum $\left(g^{\prime}-r^{\prime}\right)$ color of $\sim 0.5$ mag and an ionized-gas metallicity flattening associated with it only in the case of low-mass galaxies. Comparatively, more massive systems show a rather uniform negative metallicity gradient. The correlation between metallicity flattening and stellar mass for these systems results in $p$-values as low as 0.01 . Independent of the mechanism having shaped the outer light profiles of these galaxies, stellar migration or a previous episode of star formation in a shrinking star-forming disk, it is clear that the imprint in their ionized-gas metallicity was different for low- and high-mass Type II galaxies. In the case of Type III disks, a positive correlation between the change in color and abundance gradient is found (the null hypothesis is ruled out with a $p$-value of 0.02 ), with the outer disks of Type III galaxies with masses $\leq 10^{10} M_{\odot}$ showing a weak color reddening or even a bluing. This is interpreted as primarily due to a mass downsizing effect on the population of Type III galaxies that recently experienced an enhanced inside-out growth.
\end{abstract}

Key words. galaxies: abundances - galaxies: evolution - galaxies: photometry - galaxies: ISM - ISM: abundances - H II regions

\footnotetext{
^ Based on observations collected at the German-Spanish Astronomical Center, Calar Alto, jointly operated by the Max-Planck-Institut für Astronomie Heidelberg and the Instituto de Astrofísica de Andalucía (CSIC ).
} 


\section{Introduction}

After the pioneering works on surface photometry of nearby galaxies by Patterson (1940), de Vaucouleurs (1959), Sérsic (1968), and Freeman (1970), it became accepted that galaxy disks follow an exponential light profile. The inside-out scenario of galaxy formation predicts that the outskirts of a galaxy needs longer times for their assembly, resulting in an exponential decline of the radial light distribution and the metal abundances (White \& Frenk 1991; Mo et al. 1998). In the last two decades, especially with the advent of CCD imaging first and SDSS drift-scanning imaging more recently, we have learned that the vast majority of nearby disks show breaks ${ }^{1}$, which we call breaks hereafter. The radial position of this break should not be affected by inclination effect, as suggested by Sánchez-Blázquez et al. (2009), Martín-Navarro et al. (2014) in their surface brightness (SB) radial profiles after several scale lengths, and these can be either down- or up-bending. Erwin et al. (2005) and Pohlen \& Trujillo (2006) proposed a detailed classification of the different SB distributions in three general categories: (i) Type I (TI) profiles that follow a single exponential law beyond the bulge area along all the optical extension of the galaxies, (ii) Type II (TII) profiles that present a double exponential law with a down-bending beyond the break radius, and (iii) Type III (TIII) profiles that exhibit an up-bending in the outer part. The observational results obtained at high redshift (Pérez 2004; Azzollini et al. 2008) also suggest that breaks are present in distant galaxies and that, once formed, are long lived (Governato et al. 2007; Martínez-Serrano et al. 2009). This variety of radial morphologies have been tentatively explained by different mechanisms: outer Lindblad resonances (OLR; Martín-Navarro et al. 2012; Muñoz-Mateos et al. 2013), the presence of a bar (Debattista et al. 2006) or longlived spiral arms (Scarano et al. 2011), a shrinking of the starforming disk (Sánchez-Blázquez et al. 2009; Zheng et al. 2015, Z15 hereafter), changes in star-formation triggering mechanisms (Elmegreen \& Parravano 1994), satellite accretion or the existence of a star formation (SF) threshold radius beyond which only stellar migration would populate the outer disk (Roškar et al. 2008, R08 hereafter). The recent findings of a reddening in the optical broadband colors for 39 TII profiles (Bakos et al. 2008, B08 hereafter) have provided a fundamental piece of evidence to the actual scenario for the formation of galaxy disks and posed a challenge to these mechanisms. In addition, B08 found a characteristic minimum color associated with these U-shaped color profiles. This kind of reddening in the optical colors is better explained as being due to a shrinking of the region of where SF has taken place over time (Z15), or to stellar migration (R08). In particular, a minimum in the luminosity-weighted age (and resulting optical colors) results naturally from the theoretical predictions of the stellar migration scenario (Sánchez-Blázquez et al. 2014; R08; SB09).

A direct prediction of the somewhat naive inside-out disk-formation scenario, under the assumption of closed-box chemical enrichment, is the presence of a universal radial abundance/metallicity gradient in disk galaxies (Matteucci \& Francois 1989; Boissier \& Prantzos 2000; Muñoz-Mateos et al. 2011; Sánchez et al. 2014). This is indeed observed in most late-type galaxies for both the gas and stellar populations (e.g., Sánchez et al. 2014; González Delgado et al. 2015) but is still

\footnotetext{
Breaks and truncations are sometimes referred to as different phenomena as explained in Martín-Navarro et al. (2012). We focus our attention on the innermost change in the SB profiles happening at $\mu_{r}=22.5 \mathrm{mag} /{ }^{\prime 2}$
}

under debate whether this abundance gradient is valid for all disk galaxies and at all radii (Sánchez-Blázquez et al. 2014; Ho et al. 2015). On the other hand, not all theoretical models produce elemental abundance radial distributions as perfect exponential functions. In this regard, Mollá et al. (2015) shows how the radial distributions of oxygen abundance for a sample of theoretical galaxies with different dynamical masses is better fitted by a curve instead of only one straight line. Their distribution results flatten the inner disk, and flattens again in the outer regions of disks, mainly in the less massive galaxies. This behavior is a consequence of the ratio between the SF and the infall rates in each radial region, which, in turn, is defined by the surface stellar profile and the gas (molecular and diffuse) radial distributions. Although the surface brightness does not show any flattening associated with the oxygen abundances, colors also have a U-shape at the outer regions of disks, especially for galaxies with masses similar to the Milky Way (MW). In addition, several investigations both in our MW (Vilchez \& Esteban 1996) and in nearby galaxies (Bresolin et al. 2009, 2012; Rosales-Ortega et al. 2011; Goddard et al. 2011; Marino et al. 2012; Sánchez et al. 2012 b) have reported a shallower oxygen abundance gradient (or flattening) in the outskirts, beyond $\sim 2$ effective radii, $R_{\text {eff. }}$ In general, these deviations to the universal abundance gradient are explained in terms of variations of the in situ gas density or effective SF history, the presence of a bar, or coincidence with the corotation radius. Recently, Scarano \& Lépine (2013) showed a clear correlation between this minimum in the oxygen distribution and the corotation radii for 27 galaxies, but the mechanisms causing these different behaviors are not yet fully understood.

A fundamental question therefore arises from these results: are the breaks observed in the SB profiles and the flattening in the oxygen abundance gradients connected? In order to investigate the role of the ionized-gas metallicity in the nature of the observed changes in SB and colors, we analyze these properties in a large sample of nearby disk galaxies from the CALIFA Integral Field Spectroscopy (IFS) survey.

\section{Data and analysis}

\subsection{The sample}

We have selected the 350 galaxies observed by the CALIFA survey (Sánchez et al. 2012a) at the CAHA $3.5 \mathrm{~m}$ telescope with Potsdam Multi Aperture Spectrograph (PMAS) in the PPak mode (Kelz et al. 2006) and processed by the CALIFA v1.5 pipeline (García-Benito et al. 2015) up to September 2014. CALIFA is an IFS survey, whose main aim is to acquire spatially resolved spectroscopic information of $\sim 600$ galaxies in the Local Universe $(0.005<z<0.03)$, sampling their optical extension up to $\sim 2.5 R_{\text {eff }}$ along the major axis with a spatial resolution of FWHM 2.5" (1"/spaxel), and covering the wavelength range $3700 \AA-7500 \AA$. By construction, our sample includes galaxies of any morphological type representative of all local galaxies between $-23<M_{\mathrm{abs}, z}<-18$. Details about the data reduction are given in Husemann et al. (2013) and in García-Benito et al. (2015), and more information on the mother sample can be found in Walcher et al. (2014). We exclude from our analysis all those galaxies classified as mergers (26/350 galaxies), as interactions are expected to flatten the metallicity profiles independent of the secular mechanisms put to test in this work (e.g., Barrera-Ballesteros et al. 2015; Rupke et al. 2010; S14). Our surface-photometry sample therefore consists of 324 CALIFA galaxies. Global properties for the galaxies in our sample, such 


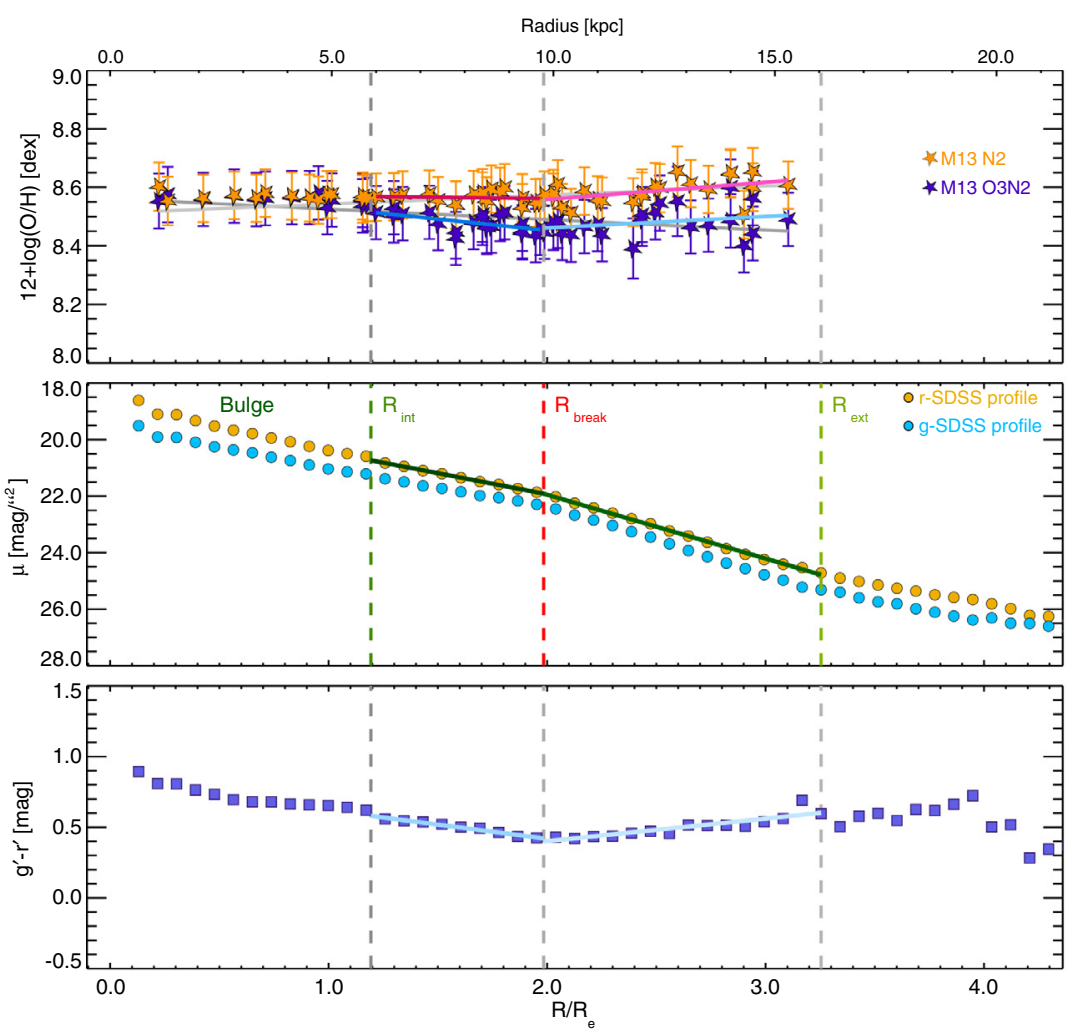

NGC5980

$$
\begin{aligned}
& z=0.0157 \\
& R_{\text {eff }}=4.93[\mathrm{kpc}] \\
& \log \left(\mathrm{M}_{*}\right)=10.73\left[\mathrm{M}_{\odot}\right] \\
& \mathrm{M}_{\mathrm{R}}=-21.81[\mathrm{mag}] \\
& \text { MorphT }=\mathrm{SbcA} \\
& \text { PhotT }=I I-C T \\
& \mu_{\mathrm{r}, \text { break }}=21.92\left[\mathrm{mag} /{ }^{\star ‘ 2}\right] \\
& \left(\mathrm{g}^{\prime}-\mathrm{r}^{\prime}\right)_{\text {break }}=0.43[\mathrm{mag}] \\
& 12+\log (\mathrm{O} / \mathrm{H})_{\text {break }}=8.56[\mathrm{dex}]
\end{aligned}
$$

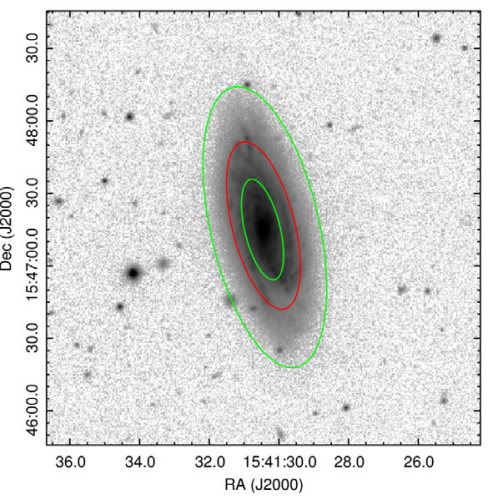

Fig. 1. Radial ionized-gas metallicity, surface brightness, and color profiles of the CALIFA disks: example for galaxy NGC5980 showing a flattening beyond the break radius and the U-shaped color profile. Left: radial oxygen abundance profile is shown in the top row; filled purple stars represent the metallicity values obtained for each H II region using the M13-O3N2 calibration (Marino et al. 2013) while the filled orange stars show those obtained via the M13-N2 calibration used in this work. The single fits of both profiles are drawn with gray solid lines. The double fits are plotted in red (inner part) and pink (outer part) for the M13-N2 case and blue (inner part) and cyan (outer part) for the M13-O3N2 case. The error bars plotted include both random and typical systematic errors associated with these calibrations. The SDSS SB profiles are shown in the second row: the $r^{\prime}$-band profile is plotted with yellow circles and the $g^{\prime}$-band profile with cyan circles. The double fit performed to the $r^{\prime}$-band is indicated with a green solid line. The third row shows the $\left(g^{\prime}-r^{\prime}\right)$ color gradient with violet squares and the double-linear fit is plotted with blue solid lines. The top $x$-axis shows the galactocentric radius in kiloparsecs, while the bottom shows the galactocentric radius normalized at $R_{\text {eff }}$. The vertical dashed (in color only in the second panel) lines denote the inner radius (green), the break radius (red) and the outer radius (green) used in each SB double fit. Right: the physical properties derived for each galaxy, including the SB type, are listed at top right, while the SDSS $3^{\prime} \times 3^{\prime} r^{\prime}$-band postage stamp image is shown at bottom right. The overplotted ellipses correspond to the same radii used for the SB double fit. (The complete atlas of the 324 CALIFA galaxies analyzed is available at https://guaix.fis.ucm.es/ raffaella/Thesis_ATLAS/CALIFA_ATLAS.pdf.)

as morphological type, stellar mass, distance, etc., were taken from Walcher et al. (2014).

\subsection{Surface brightness profiles and color gradients}

The SDSS $g^{\prime}$ and $r^{\prime}$ SB and $\left(g^{\prime}-r^{\prime}\right)$ color profiles were derived using the DR10 data products, in particular, we used the SWARP mosaicking code (Ahn et al. 2014). We selected $g^{\prime}$ - and $r^{\prime}$-band data for two reasons: (i) they are deep enough to be sensitive to the outer part of galaxies; and (ii) the breaks and corresponding U-shaped profiles were originally found in these SDSS bands (B08, Z15). We created $3^{\prime} \times 3^{\prime}$ postage stamp images (as shown in Fig. 1 for the galaxy NGC 5980) and we estimate that our SB measurements are reliable up to $27-28 \mathrm{mag} /{ }^{\prime \prime 2}$ (the DR10 images are sky subtracted contrary to the DR7 data used by B08 and our faintest SB value for this analysis ${ }^{2}$ is $27 \mathrm{mag} /{ }^{\prime \prime 2}$ ). For all galaxies in the sample we mask all contaminating sources,

\footnotetext{
2 This SB lower limit value ensures that our measurements are not affected by the contamination of the stellar halo, which starts to contribute at fainter SB, and at radii larger than $20 \mathrm{kpc}$ (Wu et al. 2002; Jablonka et al. 2010; Barker et al. 2012; Bakos \& Trujillo 2013) neither the color are affected by the extended wings of the SDSS PSF.
}

such as bright stars or background galaxies, and then we extract the flux in each band from the isophotal fitting provided by the IRAF task ELLIPSE. Each isophote was computed varying both the ellipticity $(\epsilon)$ and the position angle (PA) with a step of $1^{\prime \prime}$. This approach should have less effect on the color profiles with respect to a procedure where $\epsilon$ and PA are kept fixed. The extracted fluxes were converted to AB magnitudes and corrected for Galactic extinction using the Schlegel et al. (1998) maps. Both $g^{\prime}$ (cyan circles) and $r^{\prime}$ (yellow circles) SB profiles are plotted in Fig. 1, along with the resulting radial color profile. The details of the SB profile fitting procedure are given in Sect. 3.1.

\subsection{Oxygen abundance gradients}

We obtain spectroscopic information for $\sim 15130 \mathrm{H}$ II regions (or complexes) from our 324 CALIFA data cubes using H II EXPLORER $^{3}$. Following the prescriptions described in S14 and the analysis presented in Marino et al. (2013), we compute the radial oxygen gradients for both $\mathrm{N} 2(\log ([\mathrm{N}$ II $] \lambda 6583 / \mathrm{H} \alpha))$ and O3N2 $\left(\log (([\mathrm{O} \mathrm{III}] \lambda 5007 / \mathrm{H} \beta) / \mathrm{N} 2)\right.$ indicators normalized at $R_{\text {eff }}$

3 HIIEXPLORER: http://www.caha.es/sanchez/HII_explorer/ 
(see Fig. 1). We refer to these calibrations as M13-N2 4 and M13$\mathrm{O} 3 \mathrm{~N} 2^{5}$ hereafter, respectively. We took the disk effective radii $\left(R_{\mathrm{eff}}\right)$ values for the galaxies we analyzed from S14. For the current analysis, we use the results based on M13-N2, as it provides a better match to the abundances obtained via $T_{\mathrm{e}}$-based methods (Bresolin et al. 2012; Marino et al. 2013). Instead of a single fit (Pilyugin et al. 2014; S14), we perform two independent linear regressions in the inner and outer disk ranges to each side of the best-fitting SB break in the SDSS $r^{\prime}$-band (see Sect. 3.1). This allows us to investigate whether or not there is a connection between $\mathrm{SB}$ and $(\mathrm{O} / \mathrm{H})$ breaks using a method that is less prone to the effects of outliers and the irregular sampling of the metallicity radial distribution provided by individual $\mathrm{H}$ II regions (compared to a direct double fit of the metallicities without priors). The oxygen abundance fits are computed, including both systematic and random errors, through Monte Carlo (MC) simulations. For each galaxy within our sample, we have performed $10^{5} \mathrm{MC}$ simulations to compute the difference in slopes and its uncertainty. We assume that the line fluxes are normally distributed according their estimated uncertainty, the metallicity has an intrinsic normal scatter of $\sigma=0.0567$ [dex], and the break radius is also normally distributed. This likely overestimates the uncertainties because part of the systematics in the M13N2 calibration might come from parameters that take the same value across the disk of a given galaxy, but vary from galaxy to galaxy.

\section{Results}

\subsection{Radial profile classification}

After excluding the bulge component, we carried out a detailed analysis of the disk $g^{\prime}$ - and $r^{\prime}$-band profiles. We identified the transition radius from the bulge to the disk in the profile (innermost point of our fitting range) as that where (1) there was an evident change in the isophote's ellipticity, and (2) the brightness of the extrapolated inner disk component was equal or brighter than that of the bulge. Our procedure is aimed at deriving the broken exponential (including the position of the break radius) that best fits our SB profile via bootstrapping (see resulting best fits in the middle panel of Fig. 1). Since the position of the break radii are found to be filter-independent (B08), our profile fitting and classification was performed on the $r^{\prime}$-band data owing to their better $\mathrm{S} / \mathrm{N}$ with respect to those on the $g^{\prime}$-band (where the breaks appear brighter). In spite of that, as a consistency check we initially derived the position of the break in both bands independently, finding a very good overall agreement between the two break radii (see Fig. 2). We find that in the $r^{\prime}$-band only $16 \%$ of the CALIFA galaxies are well described by a single exponential law (TI profiles) while the remaining $84 \%$ of galaxies are better described by a broken exponential. In particular, $53 \%$ of our disks present down-bending profiles and were classified as TII, and the remaining $31 \%$ are TIII (up-bending) profiles. Previous studies by Erwin et al. (2005) and Pohlen \& Trujillo (2006; among others) have proposed that according to the presence of a bar or/and to the relative position of the break with respect to the bar, the TII class could be divided into different subgroups. The up-bending TIII breaks represent $31 \%$ of our sample and historically they are also subdivided according to the possible nature of the outer zone (spheroid or disk like outer region). Our statistical analysis is focused on the possible relation

\footnotetext{
$412+\log (\mathrm{O} / \mathrm{H})=8.743[ \pm 0.027]+0.462[ \pm 0.024] \times \mathrm{N} 2$.

$512+\log (\mathrm{O} / \mathrm{H})=8.533[ \pm 0.012]-0.214[ \pm 0.012] \times \mathrm{O} 3 \mathrm{~N} 2$.
}

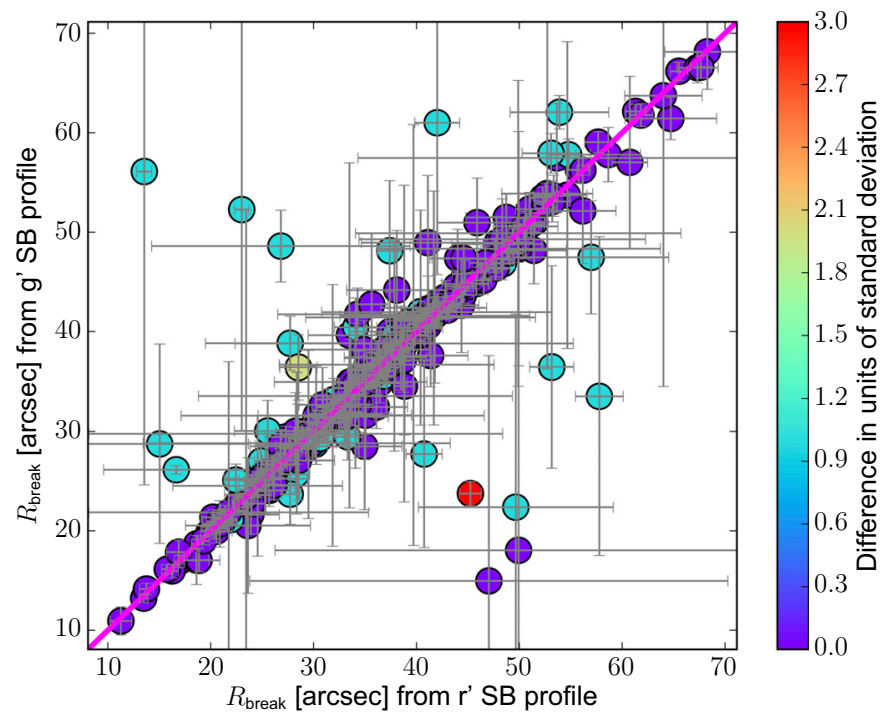

Fig. 2. Comparison of the position of the break in the surface brightness profiles derived for the SDSS $g^{\prime}$ - and $r^{\prime}$-bands for the total of 324 galaxies we analyzed in surface photometry. The color coding is shown at right and represents the offset from the 1:1 relation in units of the error of each individual point.

between the outer-disk reddening and the ionized-gas metallicity, but is not aimed at explaining in detail the physical nature of each subgroup of the TII and the TIII categories. To achieve this task, we consider only the main classes for the joint analysis of the star and gas profiles to find an easy way to compare our results with previous findings.

Finally, the results of our disks classification are presented in Table 1, including the detailed frequencies and the SB, color, and oxygen abundance measurements at break radius for each subtype. We conclude that our results are consistent with the previous classifications and that our breaks occur, as expected, at $\sim 2.5$ scale lengths (or $\sim 1.5 \times R_{\text {eff }}$ ) on average and also that the mean $\left(g^{\prime}-r^{\prime}\right)$ color at $R_{\text {break }}$ is similar to that obtained by B08 for TII disks $(\sim 0.5 \mathrm{mag}$, see Table 1$)$, although with a significant larger $(>2 \times)$ sample. The latter color value is an average observed measurement so it has not be corrected for internal reddening, which could vary as a function of the galaxy inclination.

\subsection{The interplay between stellar light and abundance profiles}

The goal of this work goes beyond the disk classification of the CALIFA galaxies. Our main aim is to find possible connections between stellar light colors and gas metallicity in the external parts of disk galaxies. To ensure a good statistical sampling, we impose that our final sample must only include spiral galaxies that have a minimum $5 \mathrm{H}$ II regions beyond the break radius and also present a broken exponential light profile (i.e., elliptical and TI galaxies are excluded from the following analysis). The final sample comprises a total of 131 galaxies (98 TII + 33TIII), which fulfill these requirements, and reduces the number of H II regions used to 8653 from the 15130 detected in the surface photometry sample. We carry out two linear regressions in the same SB intervals to calculate the difference between the slopes of the outer-to-inner color profiles, $\Delta \alpha_{\left(g^{\prime}-r^{\prime}\right)}$. As described in Sect. 2.3, we then apply the same analysis to the radial distribution of the oxygen abundance of $\mathrm{H}$ II regions and 
R. A. Marino et al.: Breaks, colors, and gas abundances within CALIFA

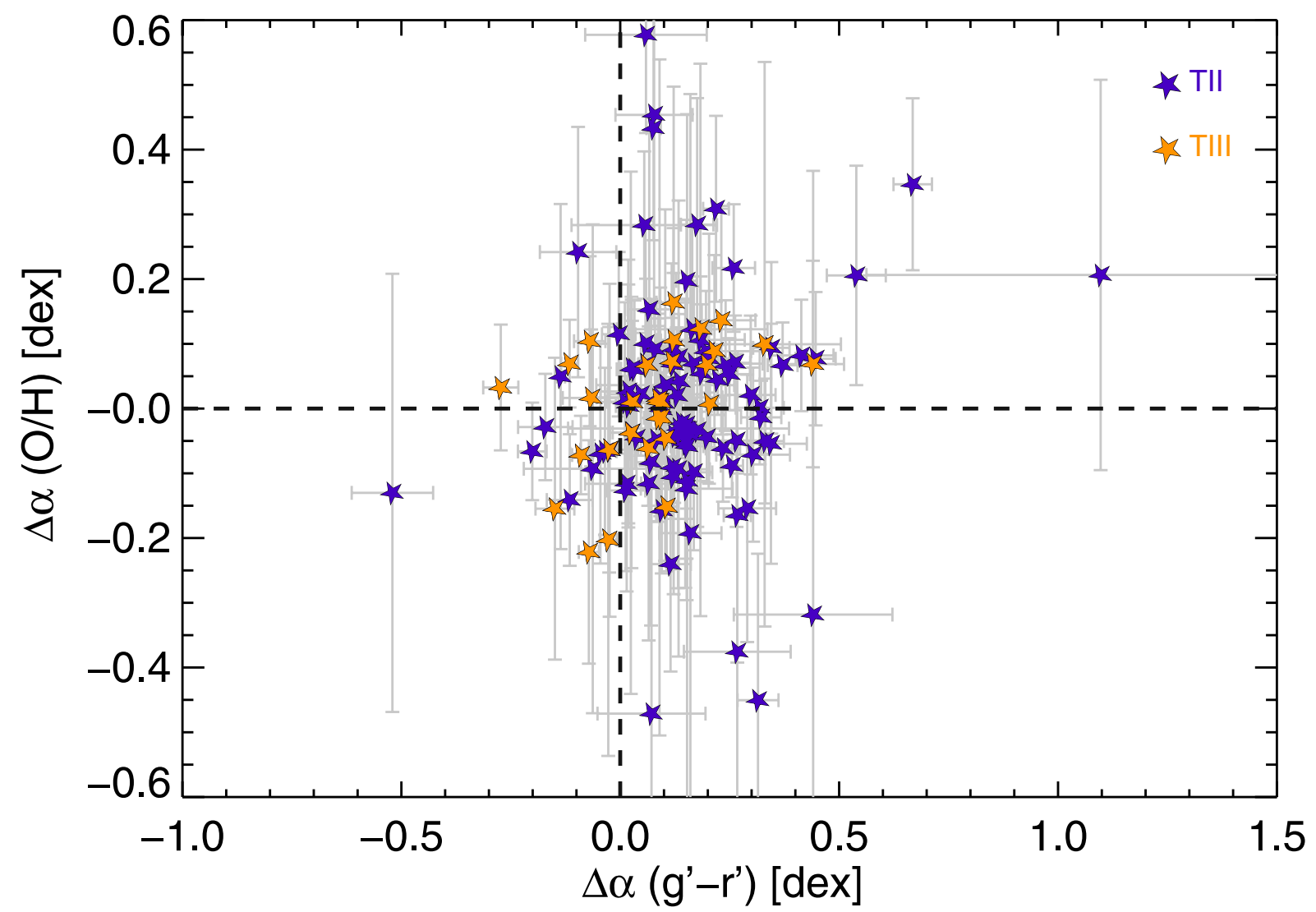

Fig. 3. Distribution of the oxygen metallicity slopes (outer-inner) difference, $\Delta \alpha_{(\mathrm{O} / \mathrm{H})}$, versus the $\left(g^{\prime}-r^{\prime}\right)$ color slopes difference, $\Delta \alpha_{\left(g^{\prime}-r^{\prime}\right)}$. We plot our TII galaxies as purple stars, while the TIII are shown in orange.

Table 1. Statistics of our sample according to the SB profiles classification.

\begin{tabular}{|c|c|c|c|c|c|c|c|}
\hline \multicolumn{8}{|c|}{ Surface-photometry sample } \\
\hline Quantities & Units & Type I & \multicolumn{3}{|c|}{$\begin{array}{l}\text { Type II } \\
\end{array}$} & \multicolumn{2}{|c|}{ Type III } \\
\hline $\begin{array}{l}\text { Number } \\
\text { Frequency }\end{array}$ & $\begin{array}{l}\text { TOT }=324 \\
{[\%]}\end{array}$ & $\begin{array}{c}53 \\
16.4 \\
\end{array}$ & \multicolumn{3}{|c|}{$\begin{array}{c}172 \\
52.8 \\
\end{array}$} & \multicolumn{2}{|c|}{$\begin{array}{c}99 \\
30.8 \\
\end{array}$} \\
\hline & & & $\mathrm{TII}_{\mathrm{C}} \mathrm{CT}^{\dagger}$ & TII.o-CT ${ }^{\dagger}$ & TII.o-OLR ${ }^{\dagger}$ & TIII-d $\mathrm{d}^{\dagger}$ & TIII-s $^{\dagger}$ \\
\hline $\begin{array}{l}\text { Number } \\
\text { Frequency } \\
\mu_{0}\end{array}$ & $\begin{array}{l}{[\#]} \\
{[\%]} \\
{\left[\mathrm{mag} /{ }^{\prime \prime 2}\right]}\end{array}$ & $\begin{array}{c}53 \\
16.4 \\
19.88 \pm 0.81 \\
\end{array}$ & $\begin{array}{r}81 \\
25.0 \\
20.14 \pm 0.61 \\
\end{array}$ & $\begin{array}{r}31 \\
9.6 \\
20.21 \pm 0.82 \\
\end{array}$ & $\begin{array}{r}60 \\
18.5 \\
20.38 \pm 0.75 \\
\end{array}$ & $\begin{array}{r}83 \\
25.6 \\
19.30 \pm 0.88 \\
\end{array}$ & $\begin{array}{r}16 \\
4.9 \\
19.49 \pm 0.61 \\
\end{array}$ \\
\hline \multicolumn{8}{|c|}{ FINAL SAMPLE } \\
\hline $\begin{array}{l}\text { Number } \\
\text { Frequency } \\
R_{\text {break }} \\
\mu_{\text {break }} \\
\left(g^{\prime}-r^{\prime}\right)_{\text {break }} \\
(12+\log (\mathrm{O} / \mathrm{H}))_{\text {break, } \mathrm{N} 2}\end{array}$ & $\begin{array}{l}\text { TOT }=131 \\
{[\%]} \\
{\left[R_{\text {eff }}\right]} \\
{\left[\mathrm{mag} /{ }^{\prime \prime 2}\right]} \\
{[\mathrm{mag}]} \\
{[\mathrm{dex} / \mathrm{kpc}]}\end{array}$ & $\begin{array}{l}\cdots \\
\cdots \\
\cdots \\
\cdots \\
\cdots \\
\cdots \\
\end{array}$ & $\begin{array}{r}37 \\
28.2 \\
1.43 \pm 0.48 \\
22.18 \pm 0.81 \\
0.52 \pm 0.11 \\
8.50 \pm 0.08 \\
\end{array}$ & $\begin{array}{r}18 \\
13.7 \\
1.43 \pm 0.37 \\
22.21 \pm 0.97 \\
0.51 \pm 0.15 \\
8.46 \pm 0.11 \\
\end{array}$ & $\begin{array}{r}43 \\
32.8 \\
1.47 \pm 0.38 \\
22.46 \pm 0.71 \\
0.51 \pm 0.13 \\
8.52 \pm 0.08 \\
\end{array}$ & $\begin{array}{r}30 \\
23.0 \\
1.50 \pm 0.49 \\
22.70 \pm 0.51 \\
0.52 \pm 0.14 \\
8.51 \pm 0.09 \\
\end{array}$ & $\begin{array}{r}3 \\
2.3 \\
1.50 \pm 0.47 \\
22.01 \pm 0.49 \\
0.75 \pm 0.19 \\
8.58 \pm 0.09 \\
\end{array}$ \\
\hline
\end{tabular}

Notes. Quantities measured at $R_{\text {break }}$ correspond to those galaxies where metallicity gradients could be measured (final sample). Errors represent the standard deviations. ${ }^{(\dagger)}$ For a detailed explanation of each category see the classification schema presented in Fig. 4 of Pohlen \& Trujillo (2006).

simultaneously fit the metallicity gradients within and beyond the $r^{\prime}$-band $R_{\text {break }}$ (obtaining the difference of the outer-to-inner oxygen slopes, $\left.\Delta \alpha_{(\mathrm{O} / \mathrm{H})}\right)$. We find a flattening or an inverted oxygen abundance trend beyond the break radius for 69/131 galaxies, which are those showing positive differences, $\Delta \alpha_{(\mathrm{O} / \mathrm{H})}>0$ (difference outer-inner). Negatives values of $\Delta \alpha_{(\mathrm{O} / \mathrm{H})}$ indicate a relative drop in the external part of the oxygen radial profile (as most profiles show a negative internal metallicity gradient). The difference between the outer and inner slopes $\left(\Delta \alpha_{(\mathrm{O} / \mathrm{H})}\right)$ of our $(\mathrm{O} / \mathrm{H})$ fits is plotted in Fig. 3 versus the color slopes, $\Delta \alpha_{\left(q^{\prime}-r^{\prime}\right)}$. In this figure, we represent the difference, $\Delta \alpha_{(\mathrm{O} / \mathrm{H})}$, of oxygen abundance slopes (outer-inner) versus the color slope difference, $\Delta \alpha_{\left(g^{\prime}-r^{\prime}\right)}$, along with their errors obtained through the propagation of the fitting uncertainties. In general, our best-fitting results are in agreement with the oxygen abundance slope distributions obtained by Sánchez et al. (2014) and Ho et al. (2015). 

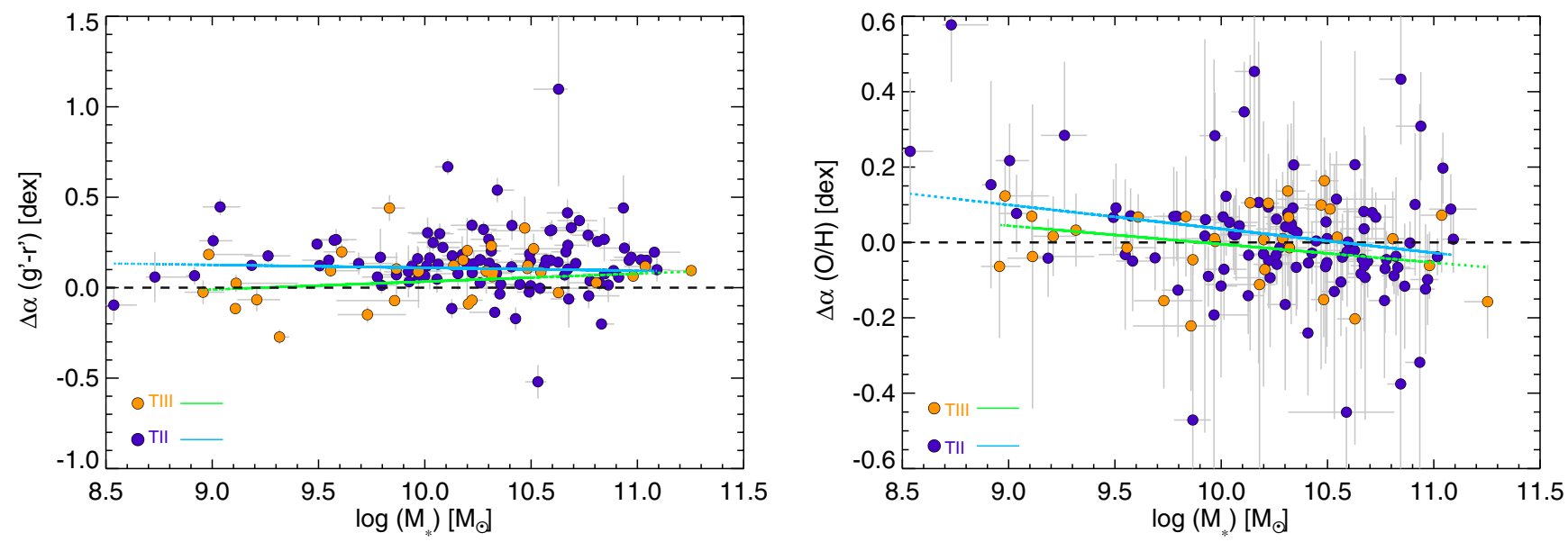

Fig. 4. Left: change in the outer-disk color gradient as a function of the galaxy stellar mass, as derived by Walcher et al. (2014), along with the linear fits to the values derived for the TII (cyan solid line) and TIII-type (green solid line) galaxies. The $p$-value parameters in this case range between 0.1 and 0.2, although the dichotomy between TII and TIII at low stellar masses is quite clear. Right: the same as above but for the change in ionized-gas metallicity gradient. The correlation in the case of the TII galaxies yields very low $p$-values, namely 0.003 (Pearson), 0.018 (Spearman), and 0.010 (Kendall), indicating that stellar mass might be one of the main drivers of the change in the ionized-gas metallicity in the outer disk of TII galaxies.

In the case of the M13-N2, our mean values for the inner and the outer slopes are -0.044 and $-0.036\left[\mathrm{dex} / R_{\mathrm{eff}}\right]$, (median -0.041 and -0.029 ), respectively.

We find that 111/131 galaxies host color profiles that present a flattening or are U-shaped. In addition, 69 of these galaxies also show a change in the outer part of their oxygen gradients $(50$ TII +19 TIII $)$. Our results suggest that U-shaped color profiles are more common in TII than TIII galaxies. However, although most TII galaxies are barred or weakly barred galaxies, no correlation is found when we analyze the behavior of the whole sample with respect to the presence or not of a bar. When all galaxies are considered together, the probability of this distribution in difference of color gradient, which is the result of a galaxy population with no change in color gradient, is smaller than $10^{-4}$ (i.e., none among the total of $10^{4} \mathrm{MC}$ runs used in this test). This result confirms the positive detection of the U-shaped color profiles within our sample. However, with regard to the change in metallicity gradient, while the distribution of gradient difference for TII disks is compatible with the null hypothesis (the Kendall test $p$-value in this case is as high as 0.3 ), in the case of the TIII disks there seems to be a correlation between color- and metallicity-gradient difference. Indeed, the $p$-value in this case for the null hypothesis is $<0.02$, so the presence of a positive correlation is supported by the data. The use of statistical tests, including nonparametrical tests, are justified as the functional dependence between these properties is not known a priori. We have used three different tests for this purpose, namely those from Pearson, Spearman, and Kendall; (see Kendall \& Gibbons 1990, and references therein). All three tests yield very similar results in all correlations analyzed.

\subsection{Mass dependence of metallicity and color breaks}

Despite TII galaxies do not show a correlation between the change in color and metallicity gradient, it is worth analyzing whether these changes might be related with other properties of the disks either global or spatially resolved. The same can be said about the TIII, where understanding the origin of the weak correlation between color and ionized-gas metallicity gradient would certainly require the analysis of other properties of these disks. Although splitting our samples of TII and TIII galaxies by physical properties would certainly benefit from an even larger sample of objects, in this section we explore the presence of potential correlations between the change in color and ionized-gas metallicity gradients with some global properties of our sample.

Due in part to the reduced size of the sample once it is split in TII and TIII-type galaxies and also to the large uncertainty of the individual measurements of the change in color and metallicity gradient only the most significant of these potential correlations would stand out. In this regard, after analyzing the relation between these changes in color and metallicity gradients with (1) presence or lack of barred structures; (2) morphological type; or (3) galaxy stellar mass, only the latter is statistically significant within our sample, and only in the case of the TII galaxies. In Fig. 4 we represent the change in color gradient (left panel) and the change in ionized-gas metallicity gradient (right panel) as a function of the stellar mass (as provided by Walcher et al. 2014). There is no clear dependence between the change in color gradient and stellar mass, despite the obvious global reddening of the outer disks in our sample. The $p$-values derived are $\sim 0.9$ (in all three tests carried out) in the case of the TII galaxies and $\sim 0.4$ in the case of the TIII systems. However, in the case of the change in ionized-gas metallicity of TII galaxies, we find that more massive systems have a rather uniform negative metallicity gradient than low-mass galaxies. Thus, at masses below $10^{10} M_{\odot}$ a drop in the outer-disk metallicity gradient is commonly found. In this case, the $p$-values found are as low as 0.003 (Pearson), 0.018 (Spearman), or 0.010 (Kendall). Again, a large number of systems, especially at the low-mass half of the distribution, would be desirable to confirm this relation. It is also worth noting that the outer-disk reddening is clear in TII galaxies at all masses, while for TIII galaxies, this is only clear at stellar masses above $10^{10} M_{\odot}$. The segregation between TII and TIII galaxies below this stellar-mass value is very clear from this figure (left panel). The best linear fit (plotted in Fig. 4) in the case of the change in the color gradients as a function of stellar masses for TII (TIII) galaxies yields 
a slope of $-0.016 \pm 0.006(0.044 \pm 0.005)\left[\mathrm{dex} / M_{\odot}\right]$. We also show in Fig. 4 the corresponding linear fit of the aforementioned correlation between the change in metallicity gradient and stellar mass in TII (TIII) galaxies in this case. The slope of the best fit is $-0.06 \pm 0.02(-0.05 \pm 0.02)\left[\mathrm{dex} / M_{\odot}\right]$.

\section{Discussion}

\subsection{Outer-disk properties}

In order to interpret the nature of SB breaks in nearby galaxies, we have carried out a joint analysis of CALIFA gas-phase metallicities and SDSS optical SB and colors. Whatever the mechanisms responsible for these breaks are, they should also be able to explain the diversity of morphologies, colors, and metallicity gradients found in these, otherwise poorly understood, outskirts of disk galaxies. Moreover, any theoretical interpretation should also explain the results derived from this work (some of them already found by other authors), namely:

(I) The percentage of SB profiles and mean break colors we found confirm those reported by previous works (Erwin et al. 2005; Pohlen \& Trujillo 2006; B08), this time using the well-defined and large sample of nearby galaxies from the CALIFA IFS survey.

(II) Most of the CALIFA TII and TIII disk galaxies show a flattening and even a reversed color gradients (see also B08).

(III) The distribution of differences in the outer-inner (gas) metallicity gradient shows no correlation with the difference in color gradient in the case of the TII disks, while there is a positive correlation between them (i.e., a metallicity flattening) in the case of the TIII disks.

(IV) The change in the ionized-gas metallicity gradient at both sides of the SB breaks in TII disk galaxies varies with the galaxy stellar mass ( $p$-value $\sim 0.01)$ in the sense that the low-mass galaxies show a more significant metallicity flattening (i.e., with respect to the inner gradient) than more massive systems.

(V) At stellar masses below $\sim 10^{10} M_{\odot}$, TII and TIII galaxies behave differently in terms of outer-disk reddening, with the latter showing little reddening or even a bluing in their color profiles.

Despite the evidence provided for the presence of these trends, the scatter is large. This suggests that each subgroup is rather inhomogeneous and therefore it likely includes galaxies with different spectrophotometric, chemical, and dynamical histories. A question naturally arises: can these observational results be reconciled in a unique disk formation theoretical scenario?

\subsection{Outer-disk formation scenarios}

The level of detail reached by recent models of galaxy formation and evolution are finally allowing us to use outer disks as laboratories for a better understanding of the relative contribution of in situ SF, stellar migration, and halo-gas and satellite accretion in shaping the observational properties of galaxy disks. Thus, Sánchez-Blázquez et al. (2009) were able to estimate that $60 \%$ of the stars in the outskirts of their simulated disk were not formed in situ, but migrated from the inner to the outer (warped) disk, leaving an important imprint on the stellar metallicity gradient. The idealized models of R08 indicate that the U-shaped and minimum in the color profile found by B08 is caused by a drop of gas surface density, mainly due to changes in the angular momentum, and that stars migrate mainly as a result of "churning" effects (Sellwood \& Binney 2002). The simulations of Minchev et al. (2012) predict that secular processes (bars and spiral structures) could redistribute material toward several disk scale lengths (up to $\sim 10 \mathrm{kpc}$ ). Recently, Z15 proposed that most of the stars currently in the outer disks of a sample of galaxies observed with Pan-STARRS1 were not formed in situ, and the pollution of their outskirts is due to the combination of radial migration plus a truncation of the SF beyond the $R_{\text {break }}$. These scenarios mainly differ in whether or not the effects of stellar migration dominate over those related with the time evolution of the size of the disk where star formation takes place.

\subsection{Implications on the evolution of disks}

Our results indicate that the majority of our disk galaxies show associated U-shaped color profiles, while more than half of them (69/131 disks in total present both features) have flat or inverted oxygen metallicity gradients. A correlation between the two is only found in the case of the TIII disks. On the other hand, TII galaxies, where the outer-disk reddening is notorious, do not follow this trend, but when a metallicity flattening is present this becomes more severe as the stellar mass decreases. In this section we explore the implications (and constraints) of these results on the different theoretical scenarios proposed.

Given the lack of correlation between the change in color and metallicity, and taken the typical sizes of our TII disks into account, we infer that the change in metallicity associated with the observed color flattening cannot be larger than $\sim 0.4$ dex or that correlation should be present. Assuming this maximum change in metallicity, we would expect a negligible change in the optical color of the stars associated with the oxygen abundance. Thus, at a fixed age and SF timescale (from instantaneous to continuous) the change in $\left(g^{\prime}-r^{\prime}\right)$ between e.g., $12+\log (\mathrm{O} / \mathrm{H})=8.3$ and 8.7 , would be smaller than $\sim 0.07$ mag (SB09; Sánchez-Blázquez et al. 2014). Hence, our results indicate that metallicity alone (at least in the ionized-gas phase) would never explain the observed outer-disk color profiles of TII galaxies. Despite the correlation found between the two quantities, even in the case of the TIII galaxies, the degree of metallicity flattening does not seem to be sufficient to explain the reddening of their outerdisk optical colors. Besides, even if a stellar metallicity gradient were present, it is not obvious that it could have an immediate effect on the ionized-gas phase abundances, especially in the case of oxygen, as this is released almost exclusively by shortlived massive stars. Therefore, we conclude that our results are in agreement with recent findings regarding positive age gradients in outer disks (Vlajić et al. 2009; Yoachim et al. 2012). In this regard, the work by González Delgado et al. (2015) on the stellar age radial profiles of 300 CALIFA galaxies (stacked by morphology and mass) has also shown a flattening of these profiles beyond 1.5-2 half-light radius (HLR). González Delgado et al. (2015) find negative extinction and stellar metallicity gradients, which leaves the age as the only possible parameter responsible for the outer-disk reddening.

Therefore, any scenario aimed at explaining the color profiles presented in this work should also predict a radial change in the luminosity-weighted age of the stellar populations in outer disks, since the radial variation of either extinction (Holwerda \& Keel 2013; González Delgado et al. 2015) or metallicity (see above) cannot be enough. In principle, both the scenario where 
the radius of the disk where in situ SF takes place shrinks with time (SB09) and the stellar migration scenario (R08) naturally predict a positive age gradient in the outer disks and are actually not mutually exclusive.

However, the use of ionized-gas metallicities could lead to more modest metallicity flattenings than those expected from the stars due to the dilution of the enriched gas by low-metallicity (or even pristine) gas from the halo (Lilly et al. 2013), preferably in the outer parts of the disk. On the other hand, this could be compensated by the fact that this halo gas might have been previously polluted by metal-rich outflows originated during early phases of star formation in the disk (Bresolin et al. 2012; Davé et al. 2011). These inflows of unpolluted gas versus enriched inflows are always there, but certainly speculative if there is no clear evidence, e.g. comparing the metallicity of the old population with the ionized gas metallicity. With the idea of overcoming those limitations, also including the potential contribution of satellite accretion to the population of the outer disks, a careful spectroscopic study of the stellar content in the outer parts of the CALIFA galaxies is being pursued by Ruiz-Lara et al. (in prep.).

Our results indicate that the interpretation of the colors and ionized-gas metallicities of outer disks might be different for TII and TIII and, possibly, for different stellar-mass ranges also. The fact that virtually all TII galaxies show a reddening in their outerdisk optical colors (independent of their stellar mass) already establishes a clear difference compared with the TIII galaxies (see below). Besides, we find that the metallicity flattening (although not correlated with the reddening in color) in TII objects is more notorious at low stellar masses, something that is less clear in the case of TIII galaxies. Finally, it is also worth keeping in mind that the mere shape of the TII profiles indicate that the amount of stars found (whose presence is ought to be explained) beyond the SB break is smaller than that in the outer-disks of TIII galaxies, at least for the intermediate-to-high stellar masses where TII and TIII galaxies show similar changes in their outer-disks color gradient (see left panel of Fig. 4).

In the case of TII galaxies, we ought to explain (1) why, for a similar level of color reddening, the outer disks of lowmass systems show a more obvious metallicity flattening than high-mass systems; but (2) the age is still the major driver of the radial change in color in either case. A possible explanation for the behavior observed in low-mass TII galaxies is the presence of radial migration possibly due to the mechanism known as "churning" (Sellwood \& Binney 2002), since these low-mass disks are expected to be kinematically cold (although some authors suggest that migration might be negligible in this case, Gogarten et al. 2010). Unfortunately, current numerical simulations do not yet allow us to establish whether this mechanism should lead to a larger radial metal diffusion, but similar outerdisk color reddening, compared with heating, which dominates the net stellar migration in more massive systems that involve a large number of galaxies under different evolutionary conditions (see SB09). One factor, which should be taken into account when considering the feasibility of these migration mechanisms as explaining the ionized-gas metal abundances of disks, is the fact that the oxygen is virtually all released by massive stars. Therefore, the oxygen abundance of the ISM should be not altered by the presence of low-mass evolving stars, which could migrate from the inner parts of the disks. However, since the oxygen abundances derived here rely on the intensity of the [NII] $6584 \AA / \mathrm{H} \alpha$ line ratio and on the empirical relation between the $\mathrm{N} / \mathrm{O}$ and $\mathrm{O} / \mathrm{H}$ abundance ratios, a flattening in nitrogen abundance (see Wu \& Zhang 2013; which could be produced in this case by migrating intermediate-mass stars) would also lead to an apparent flattening of the oxygen abundances derived. Here again, the comparison of ionized-gas and stellar abundances of outer disks could provide further clues.

According to the scenario of a shrinking star-forming disk, the stellar population in the outer disks is mainly populated as a result of in situ star formation. In that case, we would expect that the drop in surface brightness would lead to a drop in the oxygen abundance (we are very close to the Instantaneous-Recycling Approximation in this case) even if a positive color gradient is present in these regions, which is what we find for the most massive TII galaxies. Should this scenario be valid for all TII galaxies in general, we should also be able to explain why despite the drop in surface brightness we find a signal of flattening in the oxygen abundance in low-mass TII galaxies. Possible explanations could be that stellar migration also plays a role in this case (see above) or that these galaxies have experienced episodes of extended star formation, which led to oxygen enrichment, on top of a secular shrinking of the size of the disk where star formation takes place during the long quiescent episodes (see also the case of TIII disk galaxies below).

In the case of the TIII galaxies, we find (1) a correlation between outer-disk reddening and ionized-gas metallicity flattening, and (2) that galaxies with a low level of reddening (or even bluing) are typically low-mass systems. These results are compatible with a scenario where low-mass TIII galaxies are systems that have recently experienced (or are currently experiencing) an episode of enhanced inside-out growth, such as in the case of the Type-2 XUV disks (Thilker et al. 2007) with blue colors and relatively flat metallicity gradients (Bresolin et al. 2012). In low-mass galaxies the small change in the metallicity gradient across the SB break would be a consequence of their lower overall abundances and the presence of a rather homogeneous metallicity in outer disks. Indeed, recent cosmological hydrodynamical simulations by Davé et al. (2011) propose that accretion of IGM gas enriched by early outflows could be taking place in the outskirts of disks (see also Lilly et al. 2013). From the observational point of view, there are many results that show signs of accretion of metal-rich gas in the outer disks of spiral galaxies (Belfiore et al. 2015; López-Sánchez et al. 2015). Finally, we cannot exclude that a fraction of the TIII systems analyzed here could be also TI disks (which are, indeed, also growing from inside out) with only a modest change in surface brightness at the break radius position.

More massive TIII galaxies, on the other hand, show a clear outer-disk reddening and corresponding metallicity flattening (through the correlation described above). This can be explained as due to the fact that they might have experienced episodes of enhanced inside-out growth (or, equivalently, XUV emission) in their outer disks in the past. These episodes could have raised the oxygen abundance in these outer disks to the levels found in XUV disks (Bresolin et al. 2012), but which have now decreased in frequency and/or strength. This is equivalent to a shrinking in the SF disk with time having occurred in the case of the massive $\left(\geq 10^{10} M_{\odot}\right)$ disks (see Z15 and references therein). In other words, our results indicate that the outer regions of spiral disks also suffer from mass down-sizing effects. That is, at least those spiral disks that are susceptible to having experienced outer-disk growth and, therefore, get classified as TIII. It would be worth exploring whether this effect might be related to different gas fractions in the outer disks of these objects. Finally, whether stellar migration could be able to contribute significantly to the population of these shallow outer disks cannot be ruled out, at least in the case of the high-mass TIII galaxies. The former 
interpretation, however, allows us to put all TIII galaxies in the context of a common mass-driven evolutionary scenario again.

\section{Conclusions}

In this paper, we have explored the connections between the color and ionized-gas metallicity gradients in the external parts of the CALIFA disk galaxies. We find U-shaped color profiles for most TII galaxies with an average minimum $\left(g^{\prime}-r^{\prime}\right)$ color of $\sim 0.5 \mathrm{mag}$ and an associated ionized-gas metallicity flattening in the case of the low-mass galaxies. In addition, the distribution of differences in the outer-inner (gas) metallicity gradient shows no correlation with the difference in color gradient in the case of the TII disks, while there is a positive correlation between them (i.e., a metallicity flattening) in the case of the TIII disks. In the case of TIII galaxies, a positive correlation between the change in color and oxygen abundance gradient is found, with the lowmass TIII $\left(\geq 10^{10} M_{\odot}\right)$ showing a weak color reddening or even a bluing.

In the case of TII galaxies, the observed color reddening could be explained by the presence of stellar radial migration. Alternatively, within the scenario of a shrinking star-forming disk, these galaxies should have experienced episodes of extended star formation (which have led to oxygen enrichment) on top of a secular shrinking of the size of the SF disk. In the case of TIII galaxies, a scenario where low-mass galaxies have recently shown an enhanced inside-out growth is proposed to explain the overall (negative) oxygen abundance gradient and the outer-disk bluing. For more massive TIII disks, the outer color reddening associated with a flattening in their oxygen gradients can be explained as due to a past inside-out growth, which has now decreased in frequency and/or strength. Our results indicate that the outer regions of spiral disks also suffer from mass downsizing effects.

Getting further insights into these correlations requires both larger samples and stellar metallicity measurements, which will be possible with the new generation of IFS surveys under way. Our results show that the CALIFA ionized-gas metallicities alone are not enough to tackle these problems. Furthermore, deeper IFS data for both the stellar and the gas components (MUSE, Bacon et al. 2010; MaNGA, Bundy et al. 2015; SAMI, Croom et al. 2012) should be analyzed to determine the relation between outer-disk (both gas and star) metallicity gradients and galaxy global properties. The results of this analysis should allow us to establish the mechanism(s) that dominates the photometric and chemical evolution of the outskirts of disk galaxies.

Acknowledgements. We are grateful to the anonymous referee for constructive comments and suggestions. R. A. Marino is funded by the Spanish program of International Campus of Excellence Moncloa (CEI). This study makes uses of the data provided by the Calar Alto Legacy Integral Field Area (CALIFA) survey (http://www.califa.caha.es). CALIFA is the first legacy survey being performed at Calar Alto. The CALIFA collaboration would like to thank the IAA-CSIC and MPIA-MPG as major partners of the observatory, and CAHA itself, for the unique access to telescope time and support in manpower and infrastructures. The CALIFA collaboration thanks also the CAHA staff for their dedication to this project. We thank Carmen Eliche-Moral and Antonio Cava for stimulating discussions at several points in the developments of this work. We acknowledge support from the Plan Nacional de Investigación y Desarrollo funding programs, AyA2010-15081, AyA2012-30717 and AyA2013-46724P, of Spanish Ministerio de Economía y Competitividad (MINECO). A.G.d.P. acknowledges the support from the FP7 Marie Curie Actions of the European Commission, via the Initial Training Network DAGAL under REA grant agreement PITNGA-2011-289313. C.C.-T. thanks the support of the Spanish Ministerio de Educación, Cultura y Deporte by means of the FPU fellowship program. C.J.W. acknowledges support through the Marie Curie Career Integration Grant 303912.
Support for L.G. is provided by the Ministry of Economy, Development, and Tourism's Millennium Science Initiative through grant IC 120009, awarded to The Millennium Institute of Astrophysics, MAS. L.G. acknowledges support by CONIC YT through FONDECYT grant 3140566. S.F.S. thanks the CONACYT-125180 and DGAPA-IA100815 projects for providing him support in this study. J.M.A. acknowledges support from the European Research Council Starting Grant (SEDmorph; P.I. V. Wild). P.P. is supported by FCT through the Investigador FCT Contract No. IF/01220/2013 and POPH/FSE (EC) by FEDER funding through the program COMPETE. He also acknowledges support by FCT under project FCOMP-01-0124-FEDER-029170 (Reference FCT PTDC/FISAST/3214/2012), funded by FCT-MEC (PIDDAC) and FEDER (COMPETE).

\section{References}

Ahn, C. P., Alexandroff, R., Allende Prieto, C., et al. 2014, ApJS, 211, 17 Azzollini, R., Trujillo, I., \& Beckman, J. E. 2008, ApJ, 684, 1026 Bacon, R., Accardo, M., Adjali, L., et al. 2010, in SPIE Conf. Ser., 7735, 8 Bakos, J., \& Trujillo, I. 2013, Mem. Soc. Astron. It. Supp., 25, 21 Bakos, J., Trujillo, I., \& Pohlen, M. 2008, ApJ, 683, L103

Barker, M. K., Ferguson, A. M. N., Irwin, M. J., Arimoto, N., \& Jablonka, P. 2012, MNRAS, 419, 1489

Barrera-Ballesteros, J. K., García-Lorenzo, B., Falcón-Barroso, J., et al. 2015, A\&A, 582, A21

Belfiore, F., Maiolino, R., Bundy, K., et al. 2015, MNRAS, 449, 867

Boissier, S., \& Prantzos, N. 2000, MNRAS, 312, 398

Bresolin, F., Ryan-Weber, E., Kennicutt, R. C., \& Goddard, Q. 2009, ApJ, 695, 580

Bresolin, F., Kennicutt, R. C., \& Ryan-Weber, E. 2012, ApJ, 750, 122

Bundy, K., Bershady, M. A., Law, D. R., et al. 2015, ApJ, 798, 7

Croom, S. M., Lawrence, J. S., Bland-Hawthorn, J., et al. 2012, MNRAS, 421, 872

Davé, R., Finlator, K., \& Oppenheimer, B. D. 2011, MNRAS, 416, 1354

de Vaucouleurs, G. 1959, ApJ, 130, 728

Debattista, V. P., Mayer, L., Carollo, C. M., et al. 2006, ApJ, 645, 209

Elmegreen, B. G., \& Parravano, A. 1994, ApJ, 435, L121

Erwin, P., Beckman, J. E., \& Pohlen, M. 2005, ApJ, 626, L81

Freeman, K. C. 1970, ApJ, 160, 811

García-Benito, R., Zibetti, S., Sánchez, S. F., et al. 2015, A\&A, 576, A135

Goddard, Q. E., Bresolin, F., Kennicutt, R. C., Ryan-Weber, E. V., \&

Rosales-Ortega, F. F. 2011, MNRAS, 412, 1246

Gogarten, S. M., Dalcanton, J. J., Williams, B. F., et al. 2010, ApJ, 712, 858

González Delgado, R. M., García-Benito, R., Pérez, E., et al. 2015, A\&A, 581, A103

Governato, F., Willman, B., Mayer, L., et al. 2007, MNRAS, 374, 1479

Ho, I.-T., Kudritzki, R.-P., Kewley, L. J., et al. 2015, MNRAS, 448, 2030

Holwerda, B. W., \& Keel, W. C. 2013, A\&A, 556, A42

Husemann, B., Jahnke, K., Sánchez, S. F., et al. 2013, A\&A, 549, A87

Jablonka, P., Tafelmeyer, M., Courbin, F., \& Ferguson, A. M. N. 2010, A\&A, 513, A78

Kelz, A., Verheijen, M. A. W., Roth, M. M., et al. 2006, PASP, 118, 129

Kendall, M. G., \& Gibbons, J. D. 1990, Rank Correlation Methods, 5th edn. (London: Arnold)

Lilly, S. J., Carollo, C. M., Pipino, A., Renzini, A., \& Peng, Y. 2013, ApJ, 772, 119

López-Sánchez, Á. R., Westmeier, T., Esteban, C., \& Koribalski, B. S. 2015, MNRAS, 450, 3381

Marino, R. A., Gil de Paz, A., Castillo-Morales, A., et al. 2012, ApJ, 754, 61

Marino, R. A., Rosales-Ortega, F. F., Sánchez, S. F., et al. 2013, A\&A, 559, A114

Martín-Navarro, I., Bakos, J., Trujillo, I., et al. 2012, MNRAS, 427, 1102

Martín-Navarro, I., Trujillo, I., Knapen, J. H., Bakos, J., \& Fliri, J. 2014, MNRAS, 441, 2809

Martínez-Serrano, F. J., Serna, A., Doménech-Moral, M., \& DomínguezTenreiro, R. 2009, ApJ, 705, L133

Matteucci, F., \& Francois, P. 1989, MNRAS, 239, 885

Minchev, I., Famaey, B., Quillen, A. C., et al. 2012, A\&A, 548, A126

Mo, H. J., Mao, S., \& White, S. D. M. 1998, MNRAS, 295, 319

Mollá, M., Cavichia, O., Gavilán, M., \& Gibson, B. K. 2015, MNRAS, 451, 3693

Muñoz-Mateos, J. C., Boissier, S., Gil de Paz, A., et al. 2011, ApJ, 731, 10

Muñoz-Mateos, J. C., Sheth, K., Gil de Paz, A., et al. 2013, ApJ, 771, 59

Patterson, F. S. 1940, Harvard College Observatory Bulletin, 913, 13

Pérez, I. 2004, A\&A, 427, L17

Pilyugin, L. S., Grebel, E. K., Zinchenko, I. A., \& Kniazev, A. Y. 2014, AJ, 148, 134

Pohlen, M., \& Trujillo, I. 2006, A\&A, 454, 759

Rosales-Ortega, F. F., Díaz, A. I., Kennicutt, R. C., \& Sánchez, S. F. 2011, MNRAS, 415, 2439 
A\&A 585, A47 (2016)

Roškar, R., Debattista, V. P., Stinson, G. S., et al. 2008, ApJ, 675, L65

Rupke, D. S. N., Kewley, L. J., \& Barnes, J. E. 2010, ApJ, 710, L156

Sánchez, S. F., Kennicutt, R. C., Gil de Paz, A., et al. 2012a, A\&A, 538, A8

Sánchez, S. F., Rosales-Ortega, F. F., Marino, R. A., et al. 2012b, A\&A, 546, A2

Sánchez, S. F., Rosales-Ortega, F. F., Iglesias-Páramo, J., et al. 2014, A\&A, 563, A49

Sánchez-Blázquez, P., Courty, S., Gibson, B. K., \& Brook, C. B. 2009, MNRAS, 398, 591

Sánchez-Blázquez, P., Rosales-Ortega, F. F., Méndez-Abreu, J., et al. 2014, A\&A, 570, A6

Scarano, S., \& Lépine, J. R. D. 2013, MNRAS, 428, 625

Scarano, Jr., S., Lépine, J. R. D., \& Marcon-Uchida, M. M. 2011, MNRAS, 412, 1741
Schlegel, D. J., Finkbeiner, D. P., \& Davis, M. 1998, ApJ, 500, 525

Sellwood, J. A., \& Binney, J. J. 2002, MNRAS, 336, 785

Sérsic, J. L. 1968, Atlas de galaxias australes (Observatorio Astronomico, Universidad Nacional Cordoba)

Thilker, D. A., Bianchi, L., Meurer, G., et al. 2007, ApJS, 173, 538

Vilchez, J. M., \& Esteban, C. 1996, MNRAS, 280, 720

Vlajić, M., Bland-Hawthorn, J., \& Freeman, K. C. 2009, ApJ, 697, 361

Walcher, C. J., Wisotzki, L., Bekeraité, S., et al. 2014, A\&A, 569, A1

White, S. D. M., \& Frenk, C. S. 1991, ApJ, 379, 52

Wu, H., Burstein, D., Deng, Z., et al. 2002, AJ, 123, 1364

Wu, Y.-Z., \& Zhang, S.-N. 2013, MNRAS, 436, 934

Yoachim, P., Roškar, R., \& Debattista, V. P. 2012, ApJ, 752, 97

Zheng, Z., Thilker, D. A., Heckman, T. M., et al. 2015, ApJ, 800, 120 
Appendix A: CALIFA galaxies atlas
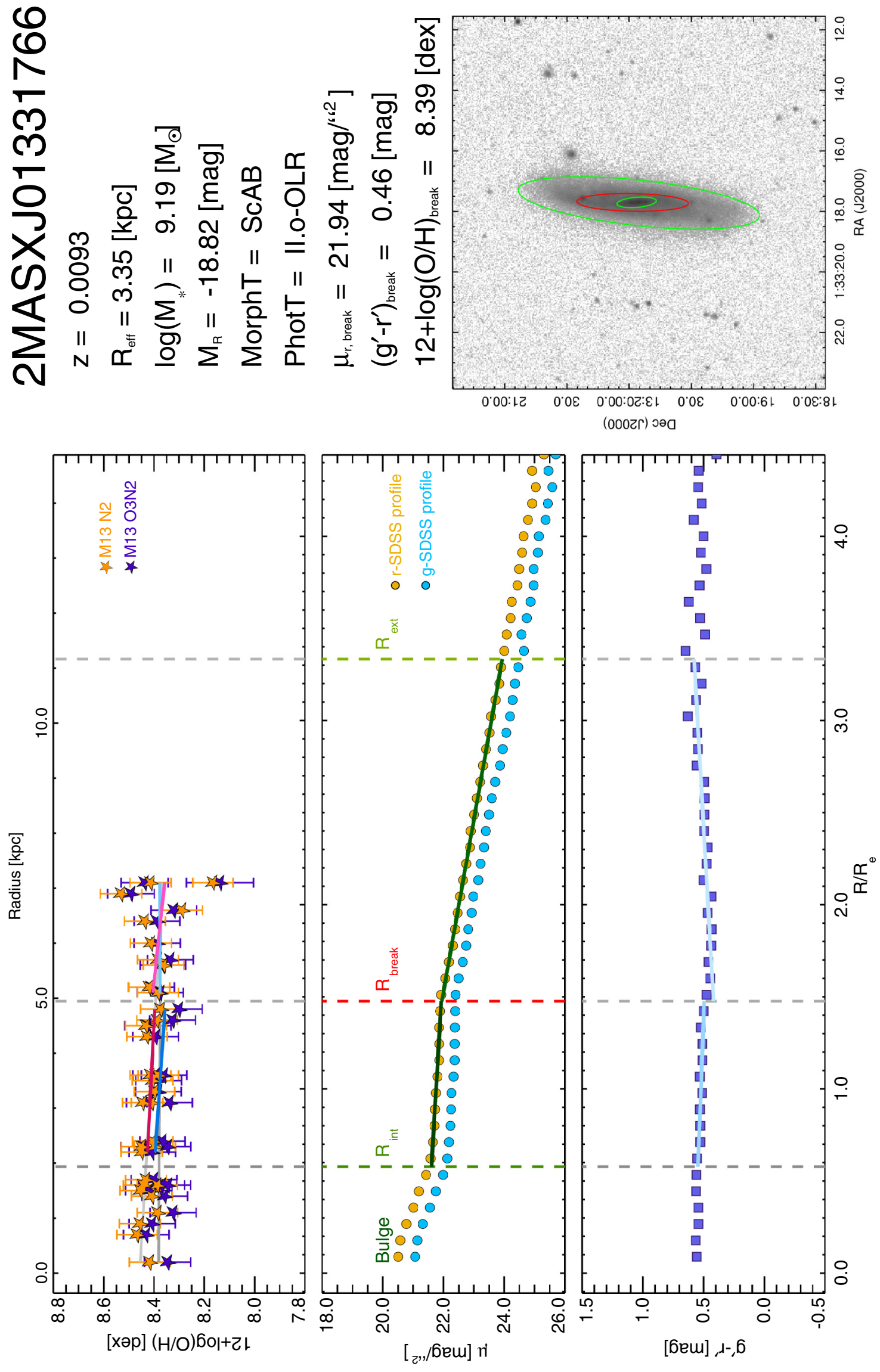

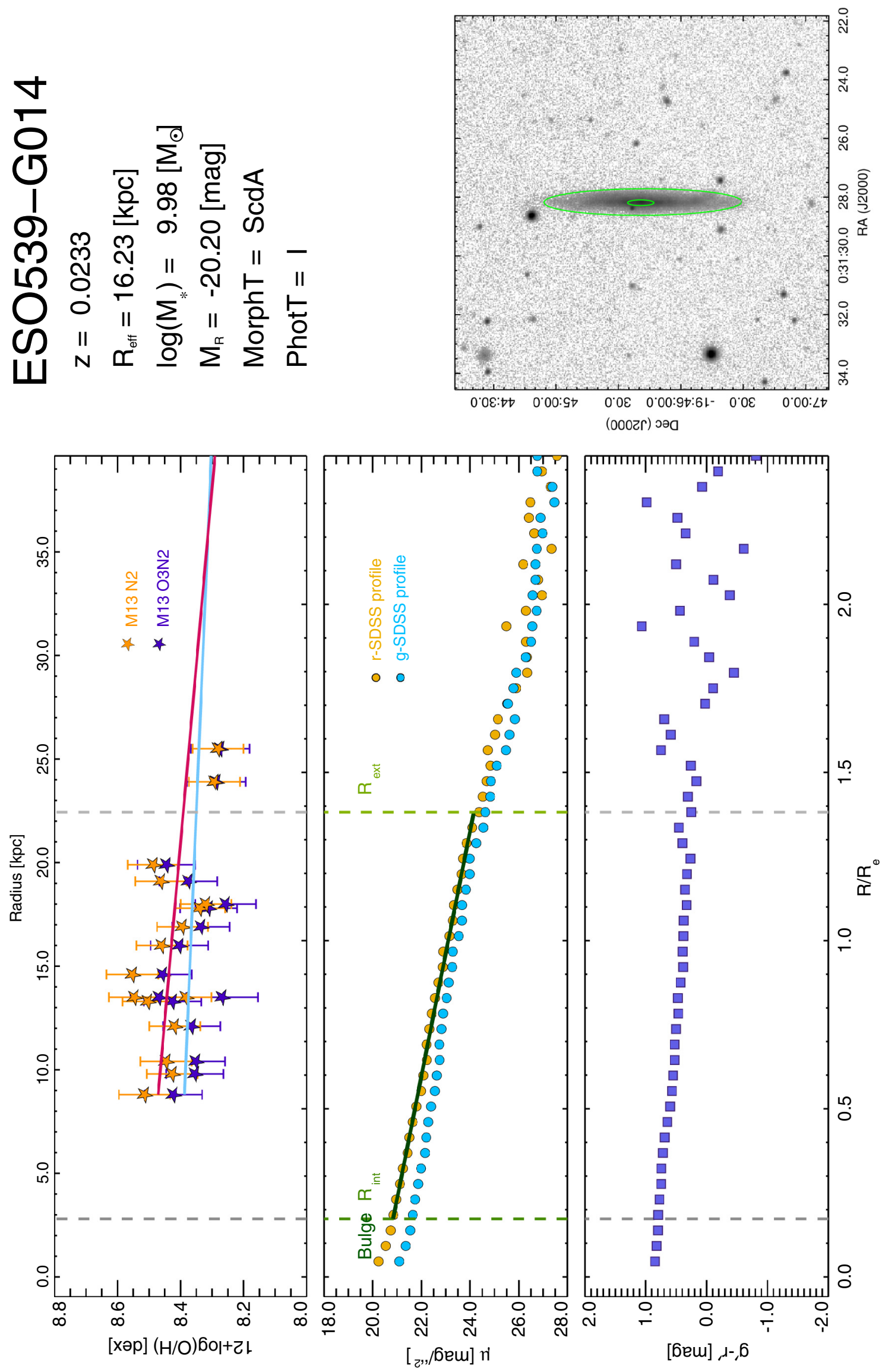
R. A. Marino et al.: Breaks, colors, and gas abundances within CALIFA
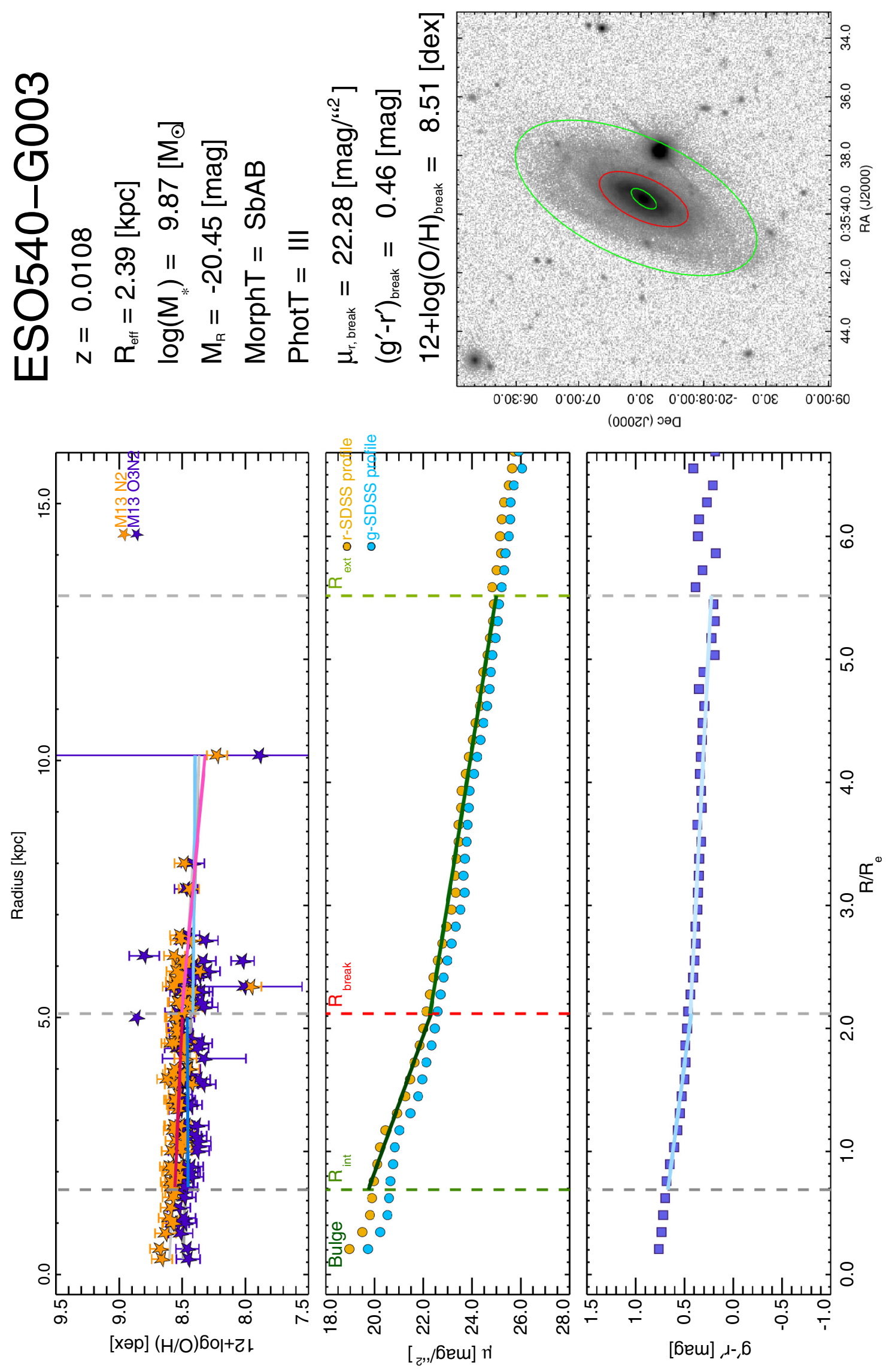

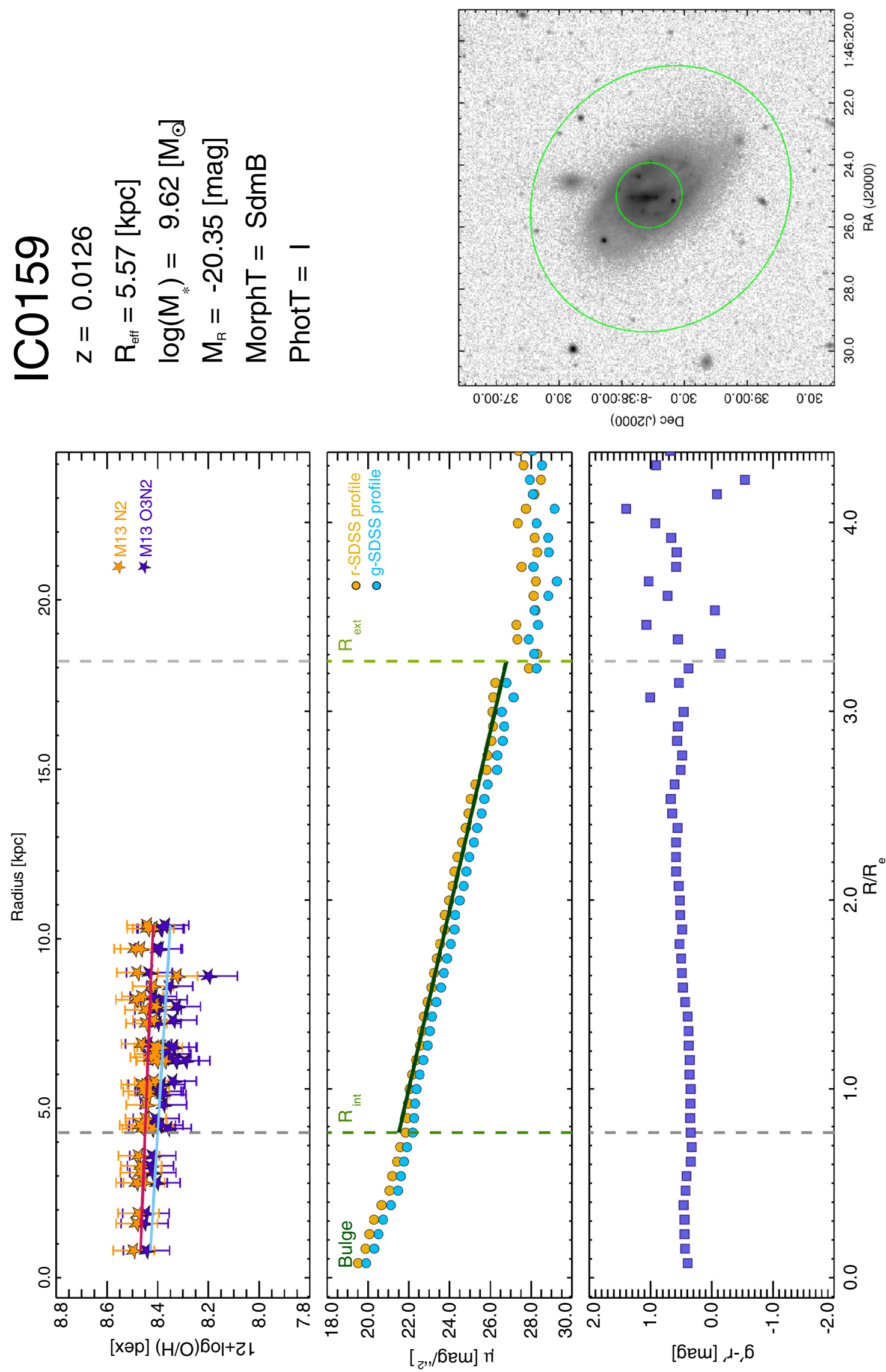
R. A. Marino et al.: Breaks, colors, and gas abundances within CALIFA
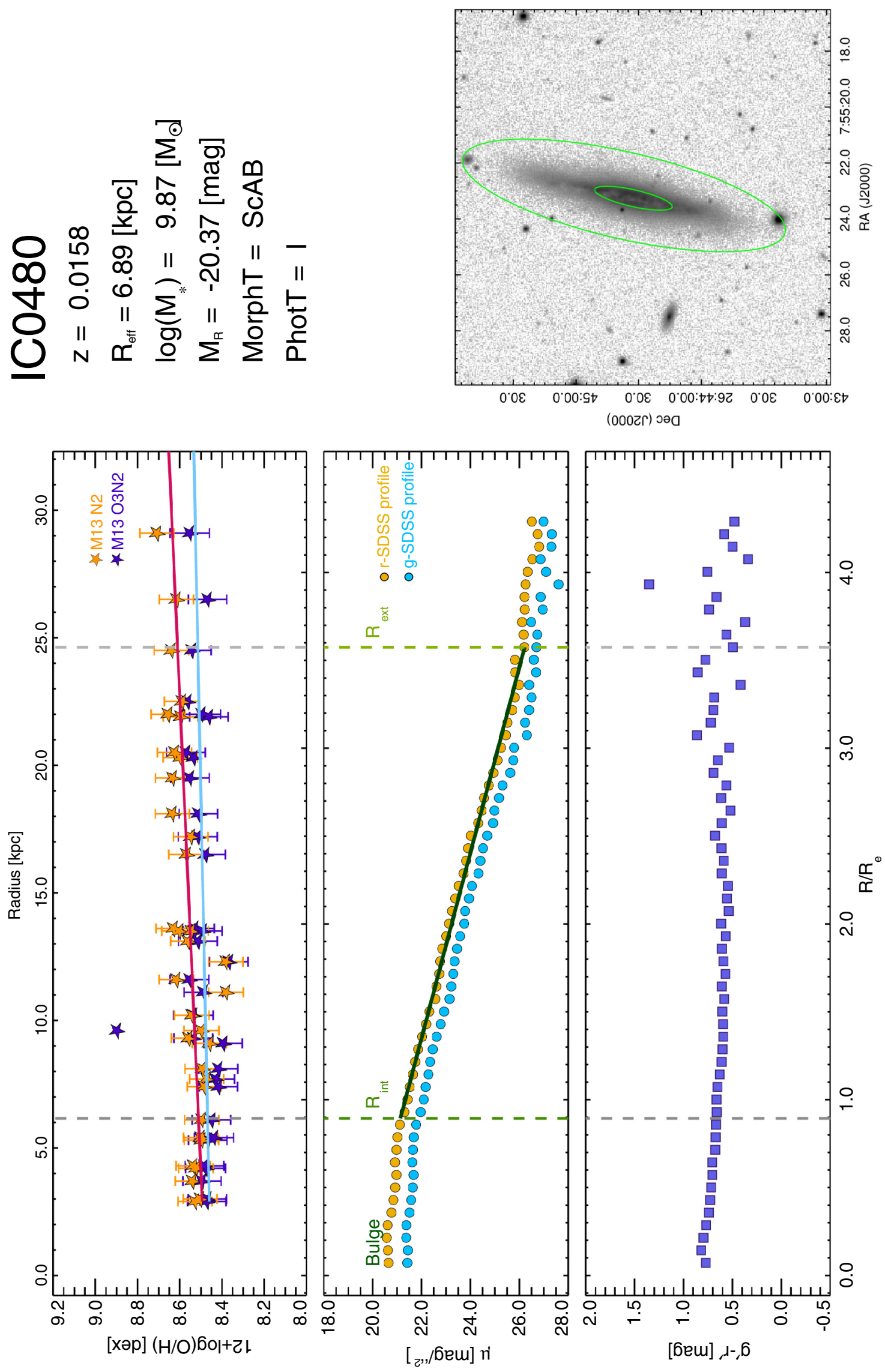

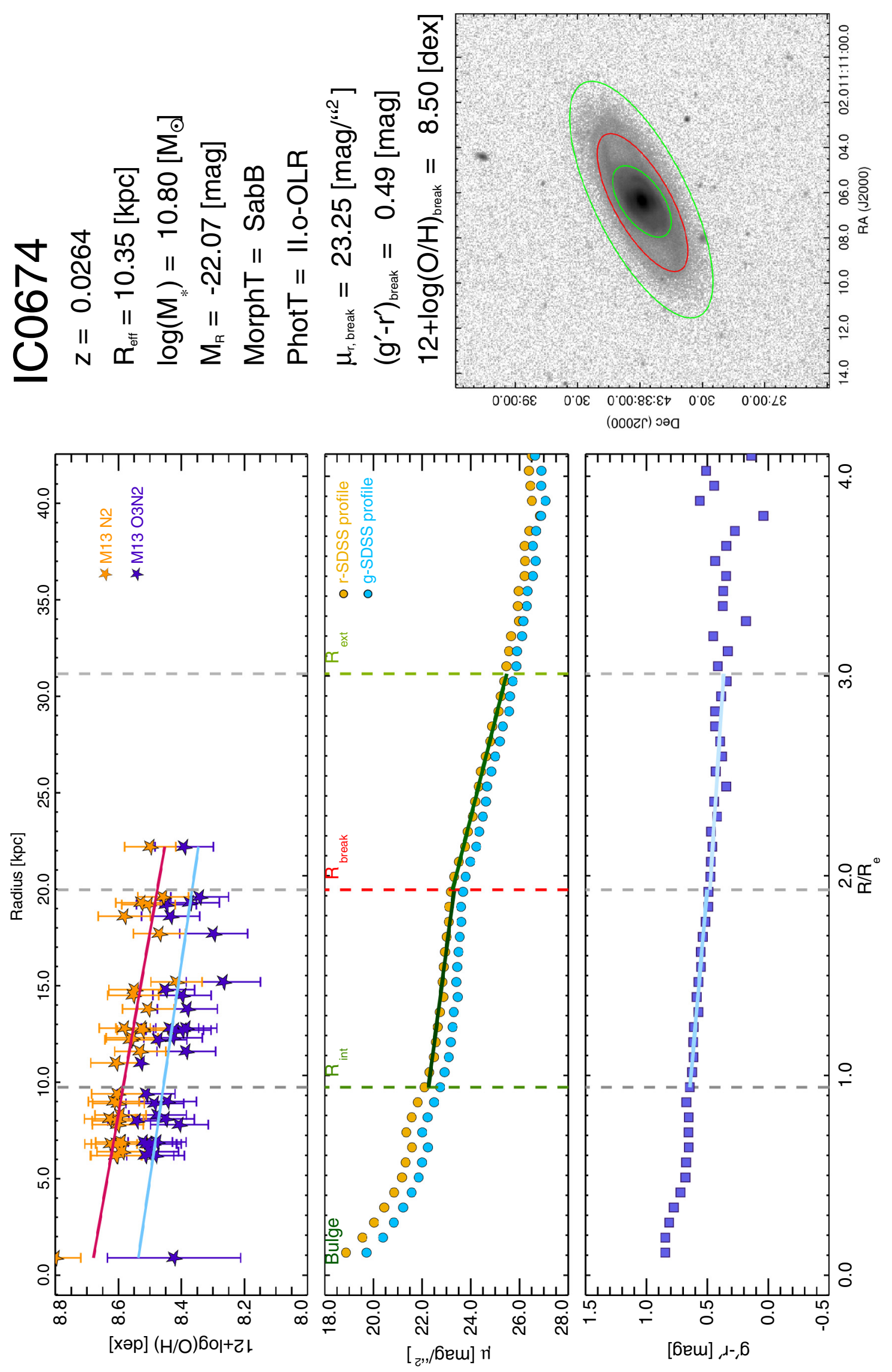

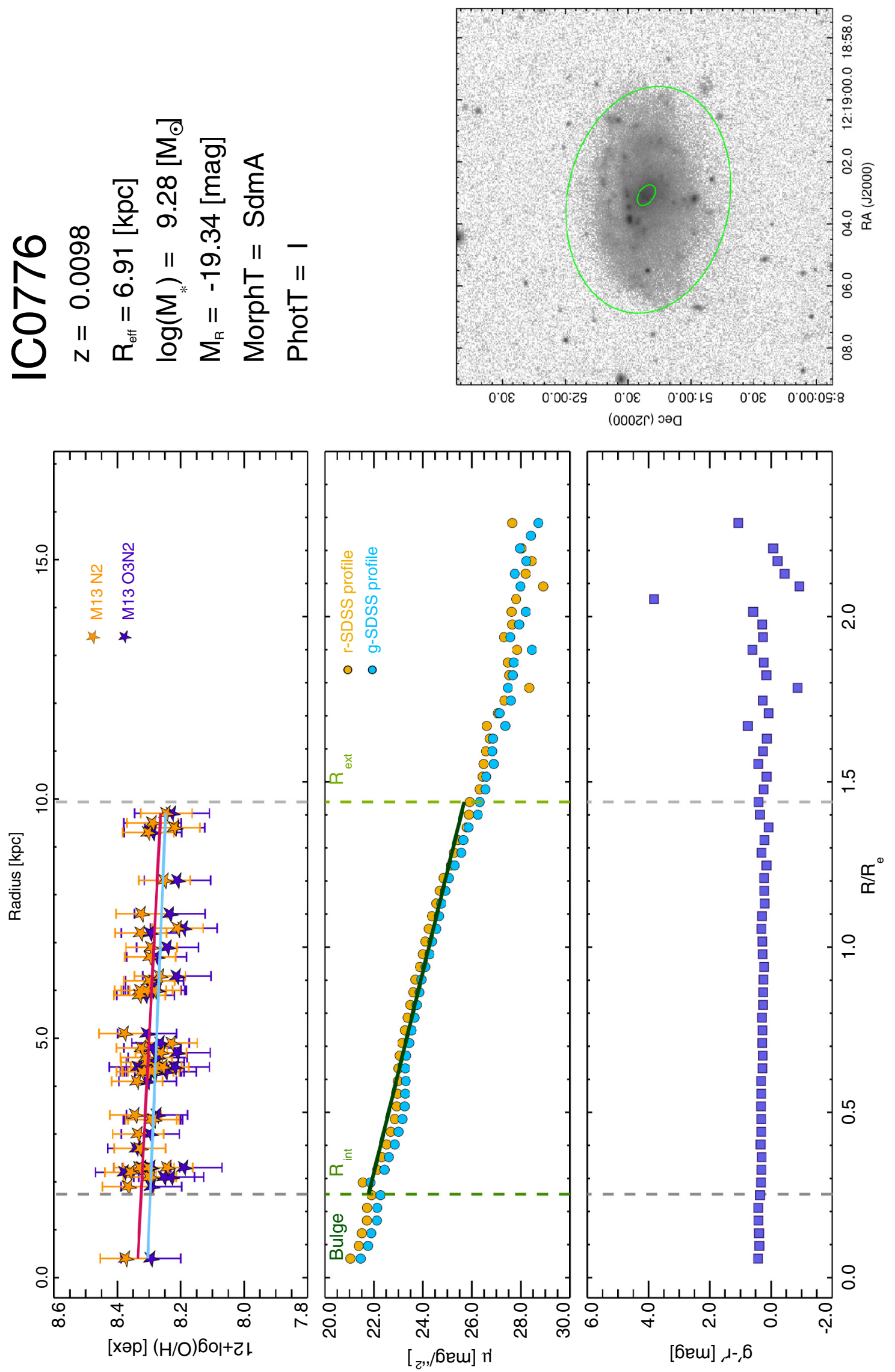

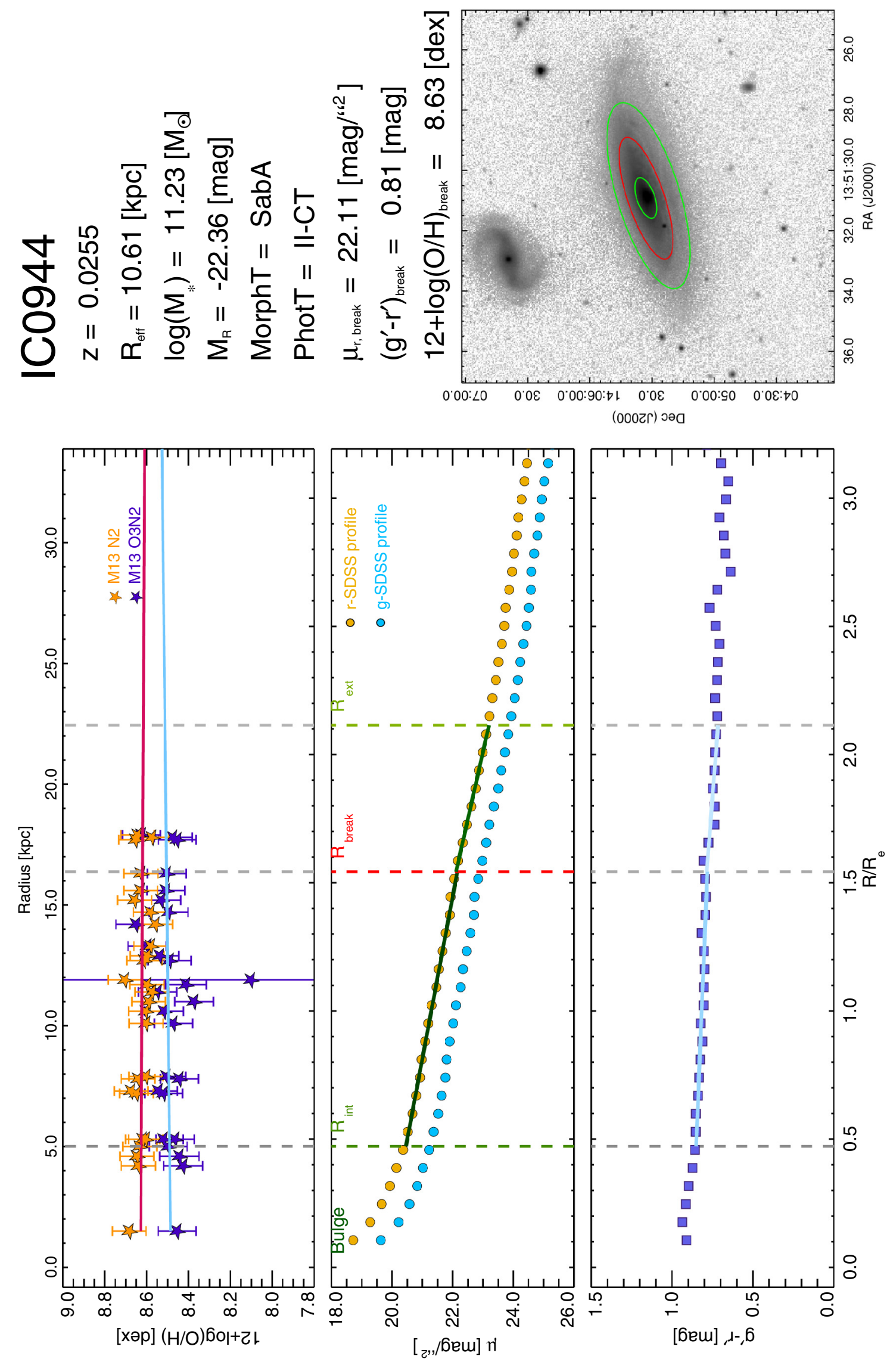

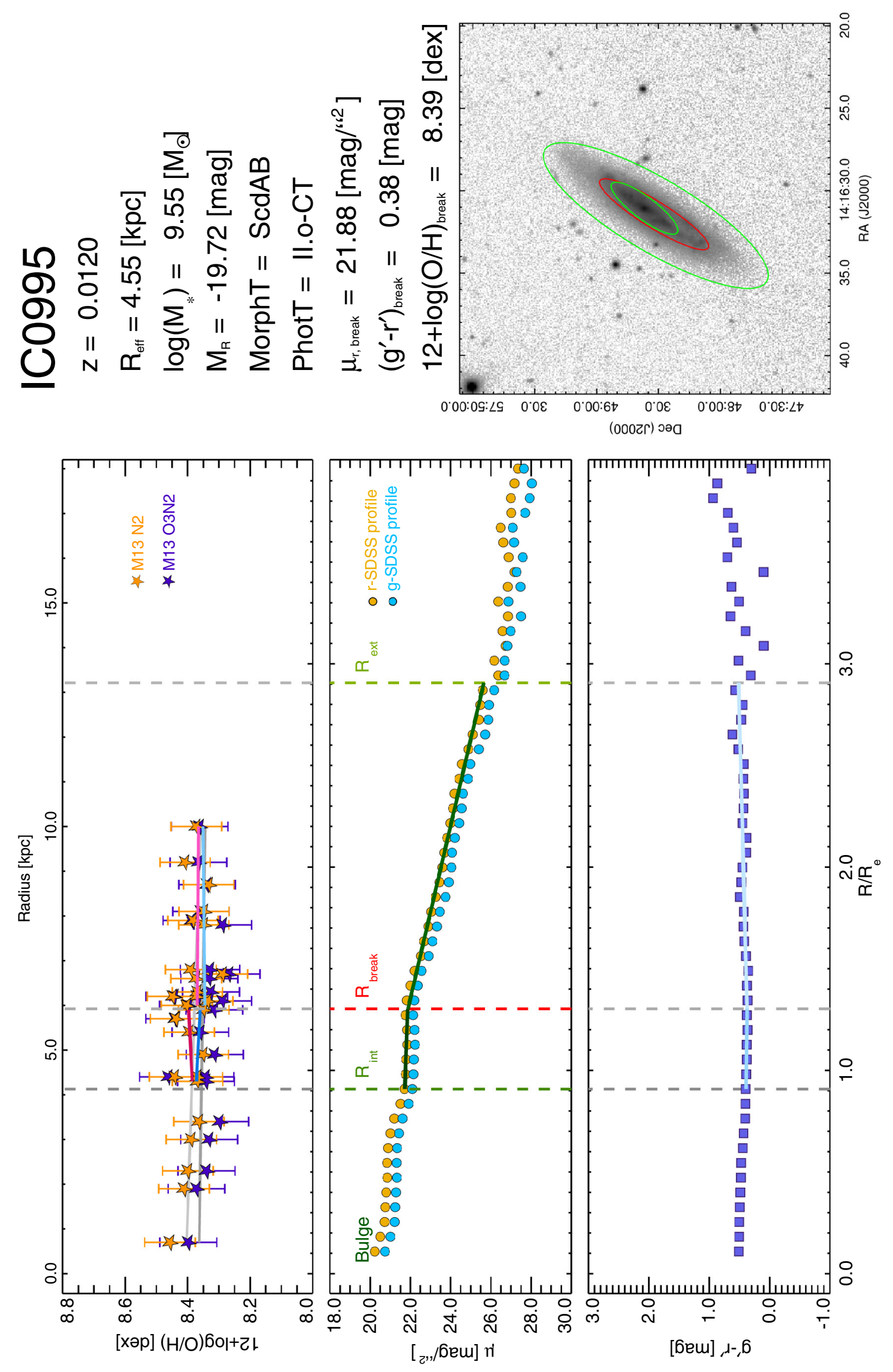

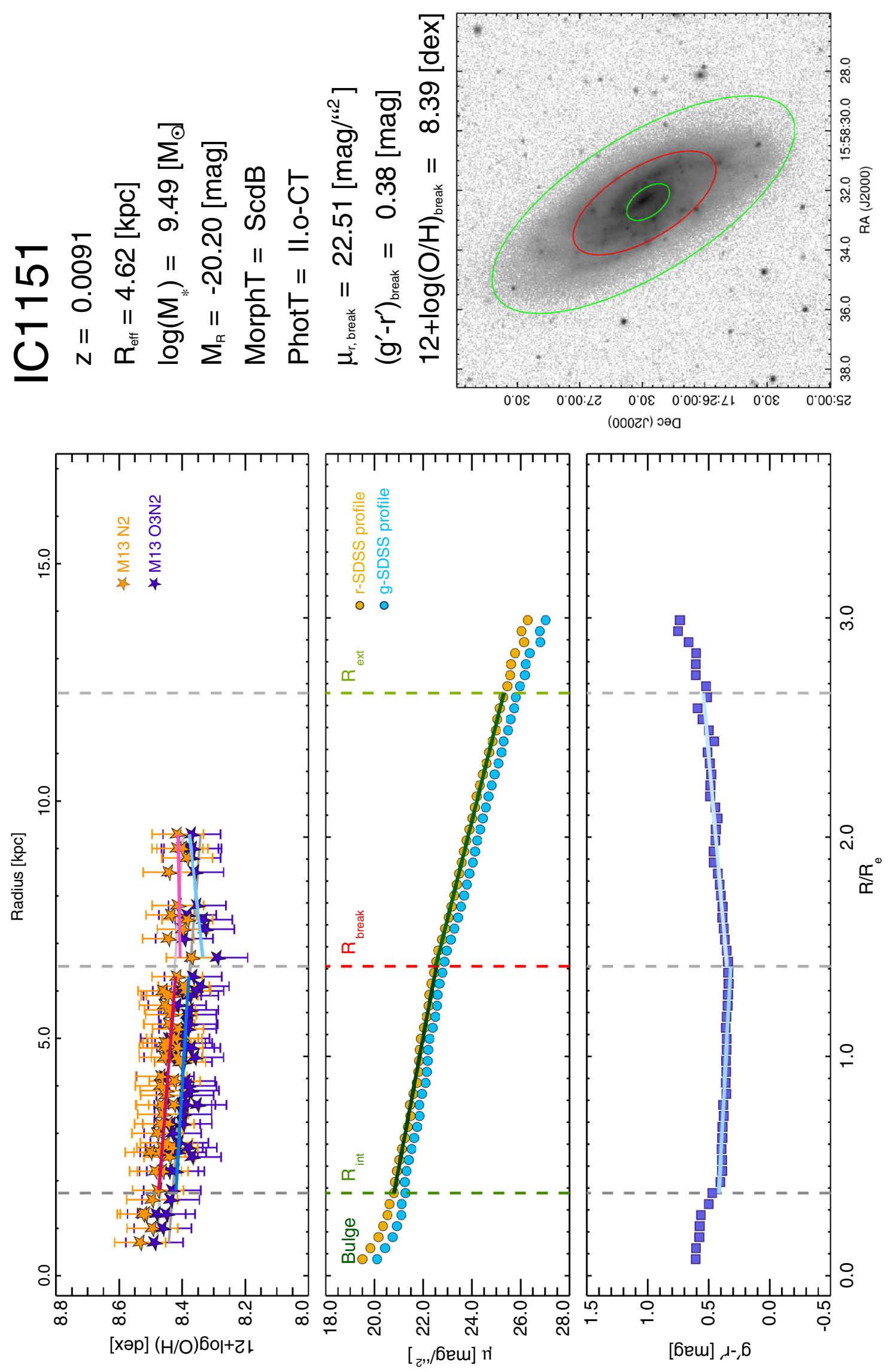


\section{Appendix B: Disk classification and physical properties of the CALIFA galaxies}

In this Appendix, we present our surface brightness classification. For each galaxy, we provide the galaxy name in the first Col. (1). In the subsequent columns, we list the following properties in order as they appear: (2) the heliocentric redshift $z$ distance obtained from the NED database; (3) Phot Type is the photometrical type obtained from our analysis of the SDSS $r^{\prime}$-band profiles; (4) the morphological type (Morph Type); (5) the stellar mass $\left(M_{*}\right)$; and (6) $R$-band absolute magnitude $\left(M_{R}\right)$ values as obtained by the CALIFA collaboration (Walcher et al. 2014), follows to the effective radius; (7) $R_{\text {eff }}$, values expressed in units of kiloparsec (Sánchez et al. 2014). Finally, the last two columns correspond to $R_{\text {rbreak }}$, the break radii obtained as the minimum of the double bootstrapping fit to the (8) SDSS $g^{\prime}$ - and (9) $r^{\prime}$-band data in units of their $R_{\text {eff }}$ along with their errors. 
A\&A 585, A47 (2016)

Table B.1. Disk classification and physical properties of the CALIFA galaxies.

\begin{tabular}{|c|c|c|c|c|c|c|c|c|}
\hline $\begin{array}{l}\text { Name } \\
\text { (1) }\end{array}$ & $\begin{array}{c}z \\
(2)\end{array}$ & $\begin{array}{l}\text { Phot type } \\
\text { (3) }\end{array}$ & $\begin{array}{l}\text { Morph type } \\
\text { (4) }\end{array}$ & $\underset{(5)}{\log \left(M_{*}\right)}$ & $\begin{array}{l}M_{R} \\
(6)\end{array}$ & $\begin{array}{l}R_{\text {eff }} \\
\text { (7) }\end{array}$ & $\begin{array}{l}R_{g^{\prime}, \text { break }} \\
\text { (8) }\end{array}$ & $\begin{array}{l}R_{r^{\prime}, \text { break }} \\
\quad(9)\end{array}$ \\
\hline 2MASXJ01331766 & 0.0093 & II.o-OLR & $\mathrm{ScAB}$ & 9.19 & -18.82 & 3.35 & $1.48 \pm 0.04$ & $1.55 \pm 0.02$ \\
\hline ESO539-G014 & 0.0233 & I & Scd A & 9.98 & -20.20 & 16.23 & $\ldots$ & $\ldots$ \\
\hline ESO540-G003 & 0.0108 & III & $\mathrm{SbAB}$ & 9.87 & -20.45 & 2.39 & $2.12 \pm 0.22$ & $2.15 \pm 0.24$ \\
\hline IC 0159 & 0.0126 & I & Sdm B & 9.62 & -20.35 & 5.57 & $\ldots$ & $\ldots$ \\
\hline IC 0480 & 0.0158 & I & $\mathrm{ScAB}$ & 9.87 & -20.37 & 6.89 & $\ldots$ & $\ldots$ \\
\hline IC 0674 & 0.0264 & II.o-OLR & Sab B & 10.80 & -22.07 & 10.35 & $1.93 \pm 0.03$ & $1.94 \pm 0.05$ \\
\hline IC 0776 & 0.0098 & I & Sdm A & 9.28 & -19.34 & 6.91 & $\ldots$ & $\ldots$ \\
\hline IC 0944 & 0.0255 & II-CT & Sab A & 11.23 & -22.36 & 10.61 & $1.54 \pm 0.05$ & $1.55 \pm 0.06$ \\
\hline IC 0995 & 0.0120 & II.o-CT & ScdAB & 9.55 & -19.72 & 4.55 & $1.30 \pm 0.04$ & $1.31 \pm 0.03$ \\
\hline IC 1151 & 0.0091 & II.o-CT & Scd B & 9.49 & -20.20 & 4.62 & $1.41 \pm 0.03$ & $1.38 \pm 0.03$ \\
\hline IC 1199 & 0.0179 & II.o-OLR & $\mathrm{SbAB}$ & 10.53 & -21.73 & 11.00 & $0.80 \pm 0.04$ & $0.74 \pm 0.05$ \\
\hline IC 1256 & 0.0175 & I & $\mathrm{SbAB}$ & 10.25 & -21.32 & 5.82 & $\ldots$ & $\ldots$ \\
\hline IC 1528 & 0.0125 & II.o-CT & SbcAB & 10.06 & -20.93 & 6.79 & $2.01 \pm 0.07$ & $1.96 \pm 0.57$ \\
\hline IC 1683 & 0.0159 & III & SbAB & 10.46 & -21.06 & 4.79 & $1.76 \pm 0.09$ & $1.66 \pm 0.11$ \\
\hline IC 1755 & 0.0257 & II-CT & $\mathrm{Sb} \mathrm{A}$ & 10.84 & -21.69 & 9.69 & $1.73 \pm 0.08$ & $1.70 \pm 0.10$ \\
\hline IC 2095 & 0.0093 & II.o-CT & $\mathrm{ScAB}$ & 8.54 & -17.84 & 4.92 & $1.18 \pm 0.05$ & $1.15 \pm 0.05$ \\
\hline IC 2101 & 0.0148 & I & ScdAB & 10.18 & -20.90 & 6.56 & $\ldots$ & $\ldots$ \\
\hline IC 2247 & 0.0150 & II-CT & Sab A & 10.45 & -20.76 & 6.97 & $1.60 \pm 0.04$ & $1.58 \pm 0.05$ \\
\hline IC 2487 & 0.0156 & II.o-OLR & $\mathrm{ScAB}$ & 10.24 & -21.03 & 7.21 & $1.57 \pm 0.05$ & $1.65 \pm 0.04$ \\
\hline IC 3598 & 0.0274 & II.o-OLR & SOAB & 10.89 & -21.81 & 11.29 & $1.65 \pm 0.06$ & $1.71 \pm 0.09$ \\
\hline IC 4215 & 0.0150 & III & $\mathrm{Sb} \mathrm{A}$ & 10.41 & -20.56 & 4.46 & $1.38 \pm 0.04$ & $1.39 \pm 0.03$ \\
\hline IC 4566 & 0.0210 & III & $\mathrm{Sb} \mathrm{B}$ & 10.82 & -21.91 & 8.78 & $1.31 \pm 0.03$ & $1.37 \pm 0.05$ \\
\hline IC 5309 & 0.0140 & III & $\mathrm{ScAB}$ & 10.22 & -20.59 & 3.38 & $2.29 \pm 0.10$ & $2.34 \pm 0.09$ \\
\hline IC 5376 & 0.0166 & II-CT & $\mathrm{Sb} \mathrm{A}$ & 10.50 & -21.07 & 7.38 & $1.57 \pm 0.05$ & $1.57 \pm 0.05$ \\
\hline MCG-01-01-012 & 0.0189 & III & SabAB & 10.77 & -20.99 & 4.19 & $3.07 \pm 0.37$ & $4.75 \pm 0.22$ \\
\hline MCG-01-54-016 & 0.0104 & II-CT & Scd A & 8.79 & -18.32 & 2.60 & $2.56 \pm 0.05$ & $2.54 \pm 0.05$ \\
\hline MCG-02-02-030 & 0.0115 & II.o-OLR & $\mathrm{SbAB}$ & 10.30 & -20.92 & 4.06 & $1.19 \pm 0.06$ & $1.20 \pm 0.06$ \\
\hline MCG-02-02-040 & 0.0117 & II.o-CT & ScdAB & 9.97 & -20.20 & 3.48 & $2.23 \pm 0.06$ & $2.20 \pm 0.07$ \\
\hline MCG-02-03-015 & 0.0188 & II.o-OLR & SabAB & 10.63 & -21.42 & 10.49 & $1.54 \pm 0.01$ & $1.53 \pm 0.01$ \\
\hline MCG-02-51-004 & 0.0199 & II-CT & $\mathrm{Sb} \mathrm{A}$ & 10.67 & -21.59 & 10.07 & $1.02 \pm 0.07$ & $1.06 \pm 0.07$ \\
\hline NGC 0001 & 0.0150 & III & Sbc A & 10.63 & -21.70 & 5.98 & $2.40 \pm 0.20$ & $2.63 \pm 0.23$ \\
\hline NGC 0023 & 0.0150 & III & $\mathrm{Sb} \mathrm{B}$ & 10.98 & -22.44 & 8.46 & $1.50 \pm 0.04$ & $1.48 \pm 0.04$ \\
\hline NGC 0036 & 0.0197 & II.o-OLR & $\mathrm{Sb} \mathrm{B}$ & 10.82 & -22.34 & 14.66 & $1.16 \pm 0.14$ & $1.16 \pm 0.33$ \\
\hline NGC 0160 & 0.0172 & II-CT & $\mathrm{Sa} A$ & 10.92 & -22.16 & 11.29 & $1.45 \pm 0.01$ & $1.44 \pm 0.02$ \\
\hline NGC 0165 & 0.0192 & I & $\mathrm{Sb} \mathrm{B}$ & 10.52 & -21.64 & 12.81 & $\ldots$ & $\ldots$ \\
\hline NGC 0171 & 0.0129 & II.o-OLR & $\mathrm{Sb} \mathrm{B}$ & 10.45 & -21.79 & 6.53 & $1.96 \pm 0.12$ & $1.96 \pm 0.12$ \\
\hline NGC 0177 & 0.0126 & II-CT & Sab A & 10.35 & -20.69 & 7.60 & $1.22 \pm 0.05$ & $1.22 \pm 0.06$ \\
\hline NGC 0180 & 0.0172 & II.o-OLR & $\mathrm{Sb} \mathrm{B}$ & 10.66 & -22.27 & 12.62 & $1.35 \pm 0.02$ & $1.32 \pm 0.03$ \\
\hline NGC 0192 & 0.0135 & II.o-CT & SabAB & 10.85 & -21.62 & 5.61 & $0.95 \pm 0.02$ & $0.95 \pm 0.01$ \\
\hline NGC 0214 & 0.0148 & II.o-OLR & SbcAB & 10.47 & -22.11 & 6.73 & $1.95 \pm 0.02$ & $1.98 \pm 0.04$ \\
\hline NGC 0216 & 0.0051 & III & $\mathrm{Sd} \mathrm{A}$ & 9.11 & -18.90 & 2.03 & $1.87 \pm 0.08$ & $1.89 \pm 0.10$ \\
\hline NGC 0234 & 0.0145 & II.o-CT & ScAB & 10.63 & -21.92 & 6.14 & $1.38 \pm 0.05$ & $1.34 \pm 0.05$ \\
\hline NGC 0237 & 0.0136 & III & $\mathrm{Sc} B$ & 10.20 & -21.15 & 4.09 & $1.63 \pm 0.04$ & $1.62 \pm 0.03$ \\
\hline NGC 0257 & 0.0171 & II-CT & $\mathrm{Sc} \mathrm{A}$ & 10.81 & -22.11 & 8.57 & $1.21 \pm 0.03$ & $1.24 \pm 0.02$ \\
\hline NGC 0444 & 0.0158 & II-CT & Scd A & 9.80 & -20.21 & 9.07 & $1.53 \pm 0.04$ & $1.52 \pm 0.06$ \\
\hline NGC 0447 & 0.0183 & I & $\mathrm{Sa} \mathrm{B}$ & 10.95 & -22.27 & 13.35 & $\ldots$ & $\ldots$ \\
\hline NGC 0477 & 0.0193 & II.o-CT & SbcAB & 10.41 & -21.67 & 13.97 & $1.09 \pm 0.03$ & $1.10 \pm 0.03$ \\
\hline NGC 0496 & 0.0196 & II-CT & Scd A & 10.30 & -21.36 & 11.00 & $1.51 \pm 0.35$ & $1.51 \pm 0.25$ \\
\hline NGC 0551 & 0.0170 & II.o-OLR & SbcAB & 10.57 & -21.53 & 7.35 & $1.49 \pm 0.08$ & $1.44 \pm 0.04$ \\
\hline NGC 0681 & 0.0057 & III-s & $\mathrm{SaAB}$ & 10.18 & -20.69 & 3.64 & $0.96 \pm 0.12$ & $0.91 \pm 0.50$ \\
\hline NGC 0693 & 0.0051 & III & E7AB & 9.86 & -19.89 & 4.10 & $1.02 \pm 0.04$ & $1.01 \pm 0.04$ \\
\hline NGC 0716 & 0.0147 & II.o-CT & $\mathrm{SbAB}$ & 10.59 & -21.35 & 5.65 & $1.56 \pm 0.03$ & $1.56 \pm 0.03$ \\
\hline NGC 0755 & 0.0053 & III & Scd B & 9.26 & -19.38 & 3.30 & $1.61 \pm 0.04$ & $1.61 \pm 0.04$ \\
\hline NGC 0768 & 0.0227 & II.o-OLR & $\mathrm{Sc} \mathrm{B}$ & 10.36 & -21.75 & 10.42 & $1.74 \pm 0.04$ & $1.75 \pm 0.04$ \\
\hline NGC 0774 & 0.0149 & III & S0 A & 10.74 & -21.38 & 4.14 & $2.16 \pm 0.35$ & $2.16 \pm 0.32$ \\
\hline NGC 0776 & 0.0159 & II.o-OLR & $\mathrm{Sb} \mathrm{B}$ & 10.60 & -21.84 & 6.90 & $1.18 \pm 0.04$ & $1.18 \pm 0.01$ \\
\hline NGC 0825 & 0.0109 & III & $\mathrm{Sa} \mathrm{A}$ & 10.37 & -20.62 & 3.90 & $2.38 \pm 0.21$ & $2.19 \pm 0.18$ \\
\hline NGC 0932 & 0.0132 & II-CT & S0a A & 10.94 & -22.11 & 7.39 & $1.11 \pm 0.03$ & $1.10 \pm 0.04$ \\
\hline NGC 1056 & 0.0052 & III-s & $\mathrm{Sa} \mathrm{A}$ & 9.97 & -19.95 & 3.40 & $0.97 \pm 0.04$ & $0.96 \pm 0.04$ \\
\hline NGC 1093 & 0.0172 & III & Sbc B & 10.47 & -21.36 & 7.15 & $1.47 \pm 0.73$ & $1.47 \pm 0.35$ \\
\hline NGC 1167 & 0.0161 & III-s & So A & 11.30 & -22.97 & 8.99 & $1.50 \pm 0.05$ & $1.52 \pm 0.04$ \\
\hline NGC 1349 & 0.0214 & III-s & E6 A & 10.80 & -22.56 & 8.12 & $1.22 \pm 0.04$ & $2.74 \pm 1.18$ \\
\hline NGC 1542 & 0.0121 & III & SabAB & 10.30 & -20.71 & 4.32 & $3.07 \pm 1.12$ & $2.99 \pm 0.32$ \\
\hline NGC 1645 & 0.0160 & I & SOa B & 10.75 & -21.72 & 9.51 & $\ldots$ & $\ldots$ \\
\hline NGC 1677 & 0.0090 & III & ScdAB & 9.32 & -19.35 & 3.19 & $1.39 \pm 0.13$ & $1.38 \pm 0.11$ \\
\hline
\end{tabular}


Table B.1. continued.

\begin{tabular}{|c|c|c|c|c|c|c|c|c|}
\hline $\begin{array}{l}\text { Name } \\
\text { (1) }\end{array}$ & $\begin{array}{c}z \\
(2)\end{array}$ & $\begin{array}{l}\text { Phot type } \\
\text { (3) }\end{array}$ & $\begin{array}{l}\text { Morph type } \\
\text { (4) }\end{array}$ & $\underset{(5)}{\log \left(M_{*}\right)}$ & $\begin{array}{l}M_{R} \\
(6)\end{array}$ & $\begin{array}{l}R_{\text {eff }} \\
\text { (7) }\end{array}$ & $\begin{array}{l}R_{g^{\prime}, \text { break }} \\
\quad(8)\end{array}$ & $\begin{array}{l}R_{r^{\prime}, \text { break }} \\
\quad(9)\end{array}$ \\
\hline NGC 2253 & 0.0125 & III & Sbc B & 10.57 & -21.60 & 2.23 & $5.16 \pm 0.10$ & $5.15 \pm 0.11$ \\
\hline NGC 2347 & 0.0153 & III & SbcAB & 10.54 & -22.14 & 5.75 & $2.68 \pm 0.24$ & $3.13 \pm 0.09$ \\
\hline NGC 2410 & 0.0161 & II.o-CT & $\mathrm{SbAB}$ & 10.80 & -21.85 & 8.44 & $2.07 \pm 0.09$ & $1.26 \pm 0.59$ \\
\hline NGC 2449 & 0.0169 & II.o-CT & SabAB & 10.81 & -21.72 & 5.53 & $1.85 \pm 0.05$ & $1.88 \pm 0.05$ \\
\hline NGC 2486 & 0.0161 & II.o-OLR & Sab B & 10.58 & -21.30 & 8.85 & $1.31 \pm 0.05$ & $1.33 \pm 0.05$ \\
\hline NGC 2487 & 0.0168 & I & $\mathrm{Sb} B$ & 10.71 & -22.19 & 11.45 & $\ldots$ & $\ldots$ \\
\hline NGC 2604 & 0.0079 & I & $\mathrm{Sd} \mathrm{B}$ & 9.60 & -20.22 & 3.67 & $\ldots$ & $\ldots$ \\
\hline NGC 2639 & 0.0121 & III & $\mathrm{Sa} \mathrm{A}$ & 11.12 & -22.25 & 4.06 & $2.46 \pm 0.06$ & $2.50 \pm 0.06$ \\
\hline NGC 2730 & 0.0138 & II.o-OLR & Scd B & 10.01 & -20.92 & 6.82 & $1.48 \pm 0.02$ & $1.45 \pm 0.03$ \\
\hline NGC 2805 & 0.0069 & III & Sc A & 9.73 & -20.76 & 5.71 & $0.47 \pm 0.05$ & $0.45 \pm 0.04$ \\
\hline NGC 2906 & 0.0081 & I & Sbc A & 10.41 & -20.78 & 2.95 & $\ldots$ & $\ldots$ \\
\hline NGC 2916 & 0.0136 & I & Sbc A & 10.51 & -22.11 & 7.13 & $\ldots$ & $\ldots$ \\
\hline NGC 3057 & 0.0063 & I & Sdm B & 9.02 & -19.25 & 4.83 & $\ldots$ & $\ldots$ \\
\hline NGC 3106 & 0.0219 & III & Sab A & 11.03 & -22.76 & 12.04 & $1.79 \pm 0.04$ & $1.82 \pm 0.04$ \\
\hline NGC 3160 & 0.0243 & II.o-CT & SabAB & 10.81 & -21.45 & 8.27 & $1.36 \pm 1.27$ & $0.87 \pm 1.24$ \\
\hline NGC 3381 & 0.0070 & III & $\mathrm{Sd} \mathrm{B}$ & 9.56 & -20.10 & 2.21 & $1.90 \pm 0.13$ & $1.95 \pm 0.04$ \\
\hline NGC 3614 & 0.0093 & II.o-OLR & SbcAB & 9.97 & -21.08 & 7.21 & $0.99 \pm 0.32$ & $0.98 \pm 0.31$ \\
\hline NGC 3619 & 0.0067 & III-s & S0a A & 10.30 & -21.00 & 4.55 & $0.84 \pm 0.01$ & $0.85 \pm 0.02$ \\
\hline NGC 3687 & 0.0100 & II.o-OLR & $\mathrm{Sb} \mathrm{B}$ & 10.16 & -20.93 & 3.80 & $1.80 \pm 0.05$ & $1.86 \pm 0.14$ \\
\hline NGC 3811 & 0.0119 & III & Sbc B & 10.32 & -21.42 & 4.54 & $1.86 \pm 0.22$ & $2.03 \pm 0.27$ \\
\hline NGC 3815 & 0.0141 & $\mathrm{I}$ & Sbc A & 10.27 & -21.02 & 4.19 & $\ldots$ & $\ldots$ \\
\hline NGC 3991 & 0.0124 & I & Sm A & 9.26 & -20.08 & 3.23 & $\ldots$ & $\ldots$ \\
\hline NGC 3994 & 0.0120 & III & SbcAB & 10.20 & -21.21 & 3.37 & $1.85 \pm 0.02$ & $1.85 \pm 0.02$ \\
\hline NGC 4003 & 0.0235 & II.o-OLR & S0a B & 10.99 & -21.98 & 7.18 & $2.24 \pm 0.99$ & $2.24 \pm 0.96$ \\
\hline NGC 4047 & 0.0130 & III & Sbc A & 10.65 & -21.93 & 4.30 & $2.34 \pm 0.06$ & $2.38 \pm 0.08$ \\
\hline NGC 4149 & 0.0117 & II.o-OLR & $\mathrm{SaAB}$ & 10.34 & -20.62 & 2.21 & $2.20 \pm 0.08$ & $2.19 \pm 0.09$ \\
\hline NGC 4185 & 0.0148 & I & SbcAB & 10.60 & -21.96 & 9.04 & $\ldots$ & $\ldots$ \\
\hline NGC 4210 & 0.0105 & II.o-OLR & $\mathrm{Sb} B$ & 10.28 & -21.01 & 3.81 & $1.76 \pm 0.10$ & $1.78 \pm 0.13$ \\
\hline NGC 4470 & 0.0093 & III & Sc A & 9.94 & -20.72 & 2.61 & $1.93 \pm 0.25$ & $1.78 \pm 0.25$ \\
\hline NGC 4711 & 0.0154 & II-CT & Sbc A & 10.26 & -21.06 & 4.08 & $1.74 \pm 0.03$ & $1.75 \pm 0.03$ \\
\hline NGC 4956 & 0.0177 & III-s & E1 A & 10.82 & -22.26 & 5.13 & $1.93 \pm 0.11$ & $1.92 \pm 0.10$ \\
\hline NGC 4961 & 0.0103 & II.o-OLR & Scd B & 9.57 & -20.21 & 3.01 & $1.64 \pm 0.03$ & $1.66 \pm 0.03$ \\
\hline NGC 5000 & 0.0207 & III & Sbc B & 10.48 & -21.78 & 7.07 & $1.43 \pm 0.03$ & $1.46 \pm 0.03$ \\
\hline NGC 5016 & 0.0106 & I & Sbc A & 10.20 & -21.07 & 3.53 & $\ldots$ & $\ldots$ \\
\hline NGC 5056 & 0.0206 & II.o-CT & $\mathrm{ScAB}$ & 10.61 & -21.90 & 7.52 & $2.22 \pm 0.06$ & $2.21 \pm 0.06$ \\
\hline NGC 5157 & 0.0263 & I & Sab B & 11.06 & -22.48 & 9.06 & $\ldots$ & $\ldots$ \\
\hline NGC 5205 & 0.0075 & III & Sbc B & 9.94 & -20.17 & 2.54 & $2.13 \pm 0.04$ & $2.11 \pm 0.04$ \\
\hline NGC 5267 & 0.0216 & II.o-OLR & Sab B & 10.86 & -22.09 & 7.77 & $1.86 \pm 0.09$ & $1.84 \pm 0.06$ \\
\hline NGC 5289 & 0.0103 & II.o-CT & Sab A & 10.22 & -20.70 & 4.79 & $1.44 \pm 0.02$ & $1.43 \pm 0.02$ \\
\hline NGC 5320 & 0.0106 & III & SbcAB & 10.16 & -21.09 & 6.20 & $1.50 \pm 0.03$ & $1.52 \pm 0.03$ \\
\hline NGC 5376 & 0.0086 & I & $\mathrm{SbAB}$ & 0.05 & -20.86 & 2.65 & $\ldots$ & $\ldots$ \\
\hline NGC 5378 & 0.0121 & II.o-OLR & $\mathrm{Sb} \mathrm{B}$ & 10.32 & -21.22 & 5.52 & $2.16 \pm 0.15$ & $2.08 \pm 0.07$ \\
\hline NGC 5379 & 0.0071 & III & SabAB & 9.83 & -19.43 & 2.42 & $1.42 \pm 0.04$ & $1.44 \pm 0.04$ \\
\hline NGC 5402 & 0.0117 & I & Sc A & 9.70 & -19.96 & 2.33 & $\ldots$ & $\ldots$ \\
\hline NGC 5406 & 0.0192 & II.o-OLR & $\mathrm{Sb} \mathrm{B}$ & 11.02 & -22.56 & 8.55 & $1.55 \pm 0.05$ & $1.63 \pm 0.04$ \\
\hline NGC 5425 & 0.0087 & II.o-OLR & ScdAB & 9.45 & -19.54 & 2.93 & $1.80 \pm 0.04$ & $1.80 \pm 0.03$ \\
\hline NGC 5439 & 0.0082 & I & $\mathrm{Sb} \mathrm{A}$ & 9.52 & -19.26 & 1.69 & $\ldots$ & $\ldots$ \\
\hline NGC 5443 & 0.0078 & III & Sab A & 10.32 & -20.78 & 4.36 & $1.67 \pm 0.04$ & $1.70 \pm 0.03$ \\
\hline NGC 5480 & 0.0080 & III & Scd A & 10.12 & -20.79 & 2.36 & $3.35 \pm 0.20$ & $3.27 \pm 1.55$ \\
\hline NGC 5519 & 0.0271 & III & $\mathrm{Sb} B$ & 10.66 & -21.91 & 16.92 & $0.78 \pm 0.02$ & $0.78 \pm 0.01$ \\
\hline NGC 5520 & 0.0081 & I & Sbc A & 9.79 & -20.24 & 2.54 & $\ldots$ & $\ldots$ \\
\hline NGC 5522 & 0.0174 & II.o-OLR & $\mathrm{SbAB}$ & 10.69 & -21.55 & 7.96 & $1.56 \pm 0.02$ & $1.55 \pm 0.02$ \\
\hline NGC 5533 & 0.0148 & III & Sab A & 11.04 & -22.63 & 11.46 & $0.96 \pm 0.06$ & $1.01 \pm 0.06$ \\
\hline NGC 5549 & 0.0279 & I & SO A & 11.31 & -23.05 & 11.58 & $\ldots$ & $\ldots$ \\
\hline NGC 5559 & 0.0193 & II.o-OLR & $\mathrm{Sb} \mathrm{B}$ & 10.53 & -21.26 & 6.62 & $1.96 \pm 0.03$ & $1.88 \pm 0.03$ \\
\hline NGC 5587 & 0.0095 & II-CT & Sa A & 10.33 & -20.56 & 4.13 & $0.60 \pm 0.02$ & $0.60 \pm 0.02$ \\
\hline NGC 5610 & 0.0190 & II.o-OLR & $\mathrm{Sb} B$ & 10.63 & -21.84 & 15.43 & $1.08 \pm 0.03$ & $1.08 \pm 0.03$ \\
\hline NGC 5616 & 0.0300 & II.o-CT & ScAB & 11.09 & -22.52 & 17.78 & $1.20 \pm 0.12$ & $1.25 \pm 0.04$ \\
\hline NGC 5622 & 0.0147 & II-CT & Sbc A & 10.23 & -20.89 & 5.36 & $1.53 \pm 0.69$ & $1.49 \pm 0.61$ \\
\hline NGC 5623 & 0.0132 & III-S & E7 A & 10.79 & -21.70 & 5.01 & $1.40 \pm 0.04$ & $1.39 \pm 0.03$ \\
\hline NGC 5630 & 0.0108 & II.o-OLR & Sdm B & 9.51 & -20.31 & 4.20 & $1.16 \pm 0.03$ & $1.17 \pm 0.03$ \\
\hline NGC 5631 & 0.0083 & III & S0 A & 10.54 & -21.50 & 4.54 & $1.33 \pm 0.12$ & $1.33 \pm 0.08$ \\
\hline NGC 5633 & 0.0096 & III & Sbc A & 10.25 & -20.98 & 1.83 & $3.13 \pm 0.06$ & $3.13 \pm 0.05$ \\
\hline NGC 5635 & 0.0164 & II-CT & $\mathrm{Sa} \mathrm{A}$ & 11.04 & -22.33 & 8.64 & $1.20 \pm 0.05$ & $1.47 \pm 0.24$ \\
\hline NGC 5656 & 0.0125 & I & $\mathrm{Sb} \mathrm{A}$ & 0.05 & -21.66 & 3.44 & $\ldots$ & $\ldots$ \\
\hline NGC 5657 & 0.0151 & II...33 & 8.64 & $1.20 \pm 0.05$ & $1.47 \pm 0.24$ & & & \\
\hline
\end{tabular}


Table B.1. continued.

\begin{tabular}{|c|c|c|c|c|c|c|c|c|}
\hline $\begin{array}{l}\text { Name } \\
\text { (1) }\end{array}$ & $\begin{array}{c}z \\
(2)\end{array}$ & $\begin{array}{l}\text { Phot type } \\
\text { (3) }\end{array}$ & $\begin{array}{l}\text { Morph type } \\
\text { (4) }\end{array}$ & $\begin{array}{c}\log \left(M_{*}\right) \\
(5)\end{array}$ & $\begin{array}{l}M_{R} \\
(6)\end{array}$ & $\begin{array}{l}R_{\text {eff }} \\
\text { (7) }\end{array}$ & $\begin{array}{l}R_{g^{\prime}, \text { break }} \\
\text { (8) }\end{array}$ & $\begin{array}{l}R_{r^{\prime}, \text { break }} \\
\text { (9) }\end{array}$ \\
\hline NGC 5656 & 0.0125 & I & $\mathrm{Sb} \mathrm{A}$ & 0.05 & -21.66 & 3.44 & & \\
\hline NGC 5657 & 0.0151 & II.o-OLR & Sbc B & 10.34 & -21.05 & 6.00 & $1.82 \pm 0.04$ & $1.89 \pm 0.05$ \\
\hline NGC 5659 & 0.0170 & II-CT & $\mathrm{Sb} \mathrm{A}$ & 10.45 & -21.14 & 7.60 & $1.73 \pm 0.03$ & $1.70 \pm 0.02$ \\
\hline NGC 5665 & 0.0091 & I & ScAB & 10.02 & -21.09 & 3.67 & $\ldots$ & $\ldots$ \\
\hline NGC 5675 & 0.0152 & III-s & $\mathrm{SaAB}$ & 10.62 & -21.84 & 7.77 & $0.65 \pm 0.04$ & $0.60 \pm 0.09$ \\
\hline NGC 5682 & 0.0094 & I & Scd B & 9.31 & -19.43 & 4.22 & $\ldots$ & $\ldots$ \\
\hline NGC 5684 & 0.0156 & III-s & E3 A & 10.70 & -21.77 & 6.57 & $2.10 \pm 0.05$ & $2.16 \pm 0.07$ \\
\hline NGC 5714 & 0.0093 & II-CT & $\mathrm{Sb} \mathrm{A}$ & 10.06 & -20.05 & 4.70 & $1.28 \pm 0.03$ & $1.31 \pm 0.02$ \\
\hline NGC 5720 & 0.0275 & III & Sbc B & 10.85 & -22.30 & 11.03 & $1.95 \pm 0.05$ & $1.95 \pm 0.05$ \\
\hline NGC 5730 & 0.0103 & I & Scd A & 9.67 & -19.76 & 4.40 & $\ldots$ & $\ldots$ \\
\hline NGC 5731 & 0.0103 & III & $\mathrm{SdAB}$ & 8.98 & -19.56 & 3.11 & $1.40 \pm 0.06$ & $1.41 \pm 0.08$ \\
\hline NGC 5732 & 0.0144 & II-CT & Sbc A & 9.89 & -20.49 & 5.43 & $1.69 \pm 0.04$ & $1.63 \pm 0.02$ \\
\hline NGC 5735 & 0.0145 & II.o-OLR & Sbc B & 10.13 & -21.37 & 6.67 & $1.74 \pm 0.03$ & $1.69 \pm 0.05$ \\
\hline NGC 5739 & 0.0198 & III & SOaAB & 11.14 & -22.91 & 9.65 & $1.73 \pm 0.05$ & $1.76 \pm 0.05$ \\
\hline NGC 5772 & 0.0182 & III & Sab A & 10.79 & -22.15 & 8.23 & $2.11 \pm 0.04$ & $2.12 \pm 0.04$ \\
\hline NGC 5784 & 0.0197 & III & S0 A & 11.16 & -22.62 & 8.43 & $1.90 \pm 0.29$ & $2.11 \pm 0.30$ \\
\hline NGC 5829 & 0.0209 & II-CT & $\mathrm{Sc} A$ & 10.59 & -21.81 & 9.22 & $1.88 \pm 0.03$ & $1.88 \pm 0.02$ \\
\hline NGC 5876 & 0.0126 & II.o-OLR & SOa B & 10.68 & -21.35 & 8.36 & $1.31 \pm 0.05$ & $1.29 \pm 0.06$ \\
\hline NGC 5888 & 0.0308 & II.o-OLR & $\mathrm{Sb} \mathrm{B}$ & 11.15 & -22.77 & 10.21 & $1.68 \pm 0.02$ & $1.62 \pm 0.07$ \\
\hline NGC 5908 & 0.0127 & III-s & $\mathrm{Sa} \mathrm{A}$ & 11.25 & -22.16 & 4.70 & $2.59 \pm 0.09$ & $2.59 \pm 0.10$ \\
\hline NGC 5947 & 0.0214 & II.o-OLR & Sbc B & 10.54 & -21.41 & 6.79 & $1.75 \pm 0.03$ & $1.74 \pm 0.03$ \\
\hline NGC 5951 & 0.0077 & I & ScdAB & 9.68 & -20.12 & 5.14 & $\ldots$ & $\ldots$ \\
\hline NGC 5957 & 0.0078 & III & $\mathrm{Sb} B$ & 9.99 & -20.79 & 3.04 & $1.70 \pm 0.01$ & $1.72 \pm 0.03$ \\
\hline NGC 5966 & 0.0168 & III-s & E4 A & 10.73 & -22.05 & 14.17 & $0.71 \pm 0.04$ & $0.73 \pm 0.05$ \\
\hline NGC 5971 & 0.0159 & II.o-CT & $\mathrm{SbAB}$ & 10.22 & -20.83 & 5.50 & $1.58 \pm 0.02$ & $1.58 \pm 0.02$ \\
\hline NGC 5980 & 0.0157 & II-CT & Sbc A & 10.73 & -21.81 & 4.93 & $1.98 \pm 0.02$ & $2.01 \pm 0.02$ \\
\hline NGC 5987 & 0.0116 & III & $\mathrm{Sa} \mathrm{A}$ & 10.99 & -22.07 & 7.94 & $1.00 \pm 0.01$ & $1.01 \pm 0.01$ \\
\hline NGC 6004 & 0.0148 & II.o-OLR & Sbc B & 10.66 & -21.88 & 6.46 & $1.50 \pm 0.01$ & $1.49 \pm 0.01$ \\
\hline NGC 6021 & 0.0179 & III & E5 A & 10.82 & -21.82 & 5.67 & $1.11 \pm 0.07$ & $1.12 \pm 0.07$ \\
\hline NGC 6032 & 0.0163 & II.o-OLR & Sbc B & 10.26 & -21.21 & 5.94 & $1.69 \pm 0.08$ & $1.70 \pm 0.09$ \\
\hline NGC 6060 & 0.0168 & II-CT & $\mathrm{Sb} \mathrm{A}$ & 10.83 & -22.27 & 10.73 & $0.87 \pm 0.02$ & $0.90 \pm 0.01$ \\
\hline NGC 6063 & 0.0114 & II-CT & Sbc A & 9.94 & -20.54 & 5.05 & $1.20 \pm 0.02$ & $1.20 \pm 0.02$ \\
\hline NGC 6081 & 0.0194 & II-CT & SOa A & 10.97 & -21.94 & 6.67 & $1.58 \pm 0.03$ & $1.57 \pm 0.03$ \\
\hline NGC 6132 & 0.0186 & I & Sbc A & 10.14 & -21.10 & 6.67 & $\ldots$ & $\ldots$ \\
\hline NGC 6154 & 0.0216 & III & Sab B & 10.77 & -22.21 & 8.23 & $1.96 \pm 0.06$ & $1.94 \pm 0.06$ \\
\hline NGC 6155 & 0.0098 & II-CT & $\mathrm{Sc} \mathrm{A}$ & 10.13 & -20.79 & 2.85 & $2.15 \pm 0.07$ & $2.12 \pm 0.07$ \\
\hline NGC 6168 & 0.0103 & II.o-OLR & ScAB & 9.78 & -20.00 & 3.17 & $1.89 \pm 0.06$ & $1.89 \pm 0.05$ \\
\hline NGC 6186 & 0.0117 & II.o-OLR & $\mathrm{Sb} \mathrm{B}$ & 10.49 & -21.21 & 2.86 & $2.19 \pm 0.03$ & $2.18 \pm 0.04$ \\
\hline NGC 6301 & 0.0293 & II-CT & Sbc A & 10.78 & -22.66 & 14.25 & $1.21 \pm 0.06$ & $1.24 \pm 0.08$ \\
\hline NGC 6310 & 0.0128 & II-CT & $\mathrm{Sb} \mathrm{A}$ & 10.49 & -20.99 & 4.78 & $1.21 \pm 0.04$ & $1.21 \pm 0.04$ \\
\hline NGC 6314 & 0.0239 & III & Sab A & 11.13 & -22.41 & 8.42 & $0.62 \pm 0.06$ & $0.60 \pm 0.08$ \\
\hline NGC 6338 & 0.0286 & III & E5 A & 11.47 & -23.33 & 30.91 & $0.53 \pm 0.04$ & $0.52 \pm 0.03$ \\
\hline NGC 6361 & 0.0141 & II-CT & Sab A & 10.89 & -21.51 & 6.40 & $1.32 \pm 0.05$ & $1.36 \pm 0.07$ \\
\hline NGC 6394 & 0.0294 & II.o-OLR & Sbc B & 10.76 & -21.88 & 14.60 & $1.85 \pm 0.54$ & $1.87 \pm 0.16$ \\
\hline NGC 6478 & 0.0239 & II-CT & $\mathrm{Sc} A$ & 10.97 & -22.59 & 9.32 & $1.17 \pm 0.06$ & $1.22 \pm 0.03$ \\
\hline NGC 6497 & 0.0217 & II.o-OLR & Sab B & 10.93 & -22.08 & 20.05 & $0.74 \pm 0.01$ & $0.74 \pm 0.01$ \\
\hline NGC 6762 & 0.0109 & III & Sab A & 10.10 & -20.30 & 4.02 & $0.87 \pm 0.02$ & $0.87 \pm 0.02$ \\
\hline NGC 6941 & 0.0216 & I & $\mathrm{Sb} \mathrm{B}$ & 10.92 & -22.39 & 11.13 & $\ldots$ & $\ldots$ \\
\hline NGC 6978 & 0.0208 & II.o-CT & $\mathrm{SbAB}$ & 11.00 & -22.16 & 7.29 & $1.57 \pm 0.03$ & $1.57 \pm 0.03$ \\
\hline NGC 7047 & 0.0199 & II.o-OLR & Sbc B & 10.70 & -21.82 & 5.59 & $2.69 \pm 0.08$ & $2.68 \pm 0.29$ \\
\hline NGC 7311 & 0.0153 & III & $\mathrm{Sa} \mathrm{A}$ & 10.97 & -22.48 & 5.55 & $2.73 \pm 0.53$ & $1.94 \pm 1.09$ \\
\hline NGC 7321 & 0.0238 & II.o-OLR & Sbc B & 10.89 & -22.48 & 9.15 & $1.60 \pm 0.07$ & $1.60 \pm 0.05$ \\
\hline NGC 7364 & 0.0163 & III & Sab A & 10.81 & -22.03 & 5.78 & $1.40 \pm 0.35$ & $1.36 \pm 0.39$ \\
\hline NGC 7466 & 0.0249 & II-CT & Sbc A & 10.62 & -21.91 & 11.72 & $1.62 \pm 0.04$ & $1.60 \pm 0.04$ \\
\hline NGC 7489 & 0.0207 & II-CT & Sbc A & 10.34 & -22.05 & 10.15 & $1.77 \pm 0.03$ & $1.76 \pm 0.03$ \\
\hline NGC 7536 & 0.0156 & I & $\mathrm{ScAB}$ & 10.25 & -21.13 & 7.31 & $\ldots$ & $\ldots$ \\
\hline NGC 7549 & 0.0158 & III & Sbc B & 10.22 & -21.39 & 8.49 & $1.25 \pm 0.04$ & $1.25 \pm 0.03$ \\
\hline NGC 7591 & 0.0164 & II.o-OLR & Sbc B & 10.67 & -21.93 & 8.17 & $1.40 \pm 0.05$ & $1.41 \pm 0.05$ \\
\hline NGC 7608 & 0.0117 & II-CT & Sbc A & 10.03 & -20.03 & 5.45 & $1.27 \pm 0.24$ & $1.32 \pm 0.21$ \\
\hline NGC 7625 & 0.0058 & III & $\mathrm{Sa} \mathrm{A}$ & 9.89 & -20.31 & 1.75 & $3.07 \pm 0.08$ & $3.05 \pm 0.09$ \\
\hline NGC 7631 & 0.0125 & II-CT & $\mathrm{Sb} \mathrm{A}$ & 10.39 & -21.10 & 6.82 & $1.74 \pm 0.03$ & $1.70 \pm 0.02$ \\
\hline NGC 7653 & 0.0142 & II-CT & $\mathrm{Sb} \mathrm{A}$ & 10.48 & -21.62 & 6.09 & $2.05 \pm 0.04$ & $2.02 \pm 0.04$ \\
\hline NGC 7671 & 0.0137 & III-s & S0 A & 10.65 & -21.55 & 3.94 & $2.60 \pm 0.07$ & $2.60 \pm 0.10$ \\
\hline NGC 7684 & 0.0170 & II-CT & SO A & 10.93 & -21.68 & 5.25 & $2.38 \pm 0.05$ & $2.39 \pm 0.07$ \\
\hline NGC 7691 & 0.0134 & II.o-CT & Sbc B & 10.18 & -21.30 & 8.67 & $1.51 \pm 0.03$ & $1.46 \pm 0.02$ \\
\hline NGC 7716 & 0.0087 & III & $\mathrm{Sb} \mathrm{A}$ & 10.29 & -20.98 & 4.14 & $1.18 \pm 0.33$ & $1.52 \pm 0.11$ \\
\hline
\end{tabular}


Table B.1. continued.

\begin{tabular}{|c|c|c|c|c|c|c|c|c|}
\hline $\begin{array}{l}\text { Name } \\
\text { (1) }\end{array}$ & $\begin{array}{c}z \\
(2)\end{array}$ & $\begin{array}{l}\text { Phot type } \\
\text { (3) }\end{array}$ & $\begin{array}{l}\text { Morph type } \\
\text { (4) }\end{array}$ & $\underset{(5)}{\log \left(M_{*}\right)}$ & $\begin{array}{l}M_{R} \\
(6)\end{array}$ & $\begin{array}{l}R_{\text {eff }} \\
\text { (7) }\end{array}$ & $\begin{array}{l}R_{g^{\prime}, \text { break }} \\
\quad(8)\end{array}$ & $\begin{array}{l}R_{r^{\prime}, \text { break }} \\
\quad(9)\end{array}$ \\
\hline NGC 7722 & 0.0133 & I & Sab A & 10.95 & -22.02 & 8.89 & $\ldots$ & $\ldots$ \\
\hline NGC 7738 & 0.0222 & III & $\mathrm{Sb} \mathrm{B}$ & 10.88 & -22.17 & 9.09 & $1.41 \pm 0.10$ & $1.47 \pm 0.05$ \\
\hline NGC 7782 & 0.0177 & II-CT & $\mathrm{Sb} \mathrm{A}$ & 11.08 & -22.64 & 10.37 & $0.99 \pm 0.03$ & $0.99 \pm 0.04$ \\
\hline NGC 7787 & 0.0219 & III & SabAB & 10.43 & -21.11 & 8.40 & $1.30 \pm 0.07$ & $1.25 \pm 0.06$ \\
\hline NGC 7800 & 0.0060 & III & IrAB & 9.21 & -19.58 & 2.44 & $1.59 \pm 0.17$ & $1.62 \pm 0.06$ \\
\hline NGC 7819 & 0.0164 & II-CT & $\mathrm{Sc} \mathrm{A}$ & 10.09 & -21.05 & 8.16 & $1.43 \pm 0.05$ & $1.43 \pm 0.04$ \\
\hline NGC 7824 & 0.0201 & II-CT & Sab A & 11.15 & -22.24 & 11.47 & $1.57 \pm 0.03$ & $1.56 \pm 0.03$ \\
\hline SDSSJ 100141 & 0.0066 & II.o-CT & $\mathrm{SmAB}$ & 8.73 & -18.26 & 3.29 & $1.68 \pm 0.04$ & $1.71 \pm 0.04$ \\
\hline UGC 00005 & 0.0238 & II-CT & Sbc A & 10.76 & -22.09 & 9.51 & $1.68 \pm 0.08$ & $1.63 \pm 0.09$ \\
\hline UGC 00036 & 0.0206 & III & SabAB & 10.84 & -21.70 & 8.32 & $1.30 \pm 0.04$ & $1.32 \pm 0.06$ \\
\hline UGC 00139 & 0.0130 & III & Scd A & 9.61 & -20.37 & 6.57 & $1.39 \pm 0.06$ & $1.44 \pm 0.05$ \\
\hline UGC 00148 & 0.0138 & II-CT & $\mathrm{Sc} \mathrm{A}$ & 10.01 & -20.75 & 5.11 & $1.53 \pm 0.07$ & $1.56 \pm 0.05$ \\
\hline UGC 00312 & 0.0142 & II.o-OLR & $\mathrm{Sd} B$ & 9.69 & -20.67 & 6.33 & $1.79 \pm 0.13$ & $1.72 \pm 0.15$ \\
\hline UGC 00809 & 0.0138 & II-CT & Scd A & 9.58 & -19.72 & 5.09 & $1.69 \pm 0.03$ & $1.70 \pm 0.04$ \\
\hline UGC 00841 & 0.0182 & II-CT & Sbc A & 9.93 & -20.22 & 7.31 & $1.26 \pm 0.03$ & $1.37 \pm 0.09$ \\
\hline UGC 00987 & 0.0152 & III & $\mathrm{SaAB}$ & 10.55 & -21.24 & 6.39 & $1.14 \pm 0.04$ & $1.14 \pm 0.05$ \\
\hline UGC 01057 & 0.0206 & II.o-OLR & $\mathrm{ScAB}$ & 10.11 & -20.88 & 8.63 & $1.73 \pm 0.06$ & $1.73 \pm 0.04$ \\
\hline UGC 01274 & 0.0255 & II-CT & $\mathrm{SaA}$ & 10.78 & -21.81 & 9.49 & $1.43 \pm 0.04$ & $1.42 \pm 0.09$ \\
\hline UGC 01368 & 0.0262 & II-CT & Sab A & 10.79 & -21.71 & 8.91 & $1.56 \pm 0.04$ & $1.51 \pm 0.07$ \\
\hline UGC 01659 & 0.0267 & II.o-OLR & $\mathrm{ScB}$ & 10.50 & -21.69 & 13.88 & $1.31 \pm 0.03$ & $1.26 \pm 0.04$ \\
\hline UGC 01749 & 0.0261 & II.o-OLR & SabAB & 10.68 & -21.44 & 15.24 & $1.01 \pm 0.01$ & $1.01 \pm 0.02$ \\
\hline UGC 01918 & 0.0165 & II.o-OLR & $\mathrm{Sb} \mathrm{B}$ & 10.55 & -21.14 & 7.70 & $1.43 \pm 0.03$ & $1.43 \pm 0.04$ \\
\hline UGC 01938 & 0.0206 & II.o-OLR & SbcAB & 10.28 & -20.94 & 8.02 & $1.91 \pm 0.16$ & $1.75 \pm 0.12$ \\
\hline UGC 02403 & 0.0133 & III & $\mathrm{Sb} \mathrm{B}$ & 10.48 & -20.80 & 4.15 & $2.21 \pm 0.04$ & $2.22 \pm 0.05$ \\
\hline UGC 02405 & 0.0249 & II-CT & Sbc A & 10.41 & -21.72 & 11.71 & $1.07 \pm 0.05$ & $1.08 \pm 0.05$ \\
\hline UGC 02465 & 0.0165 & I & So A & 10.64 & -21.28 & 10.79 & $\ldots$ & $\ldots$ \\
\hline UGC 03038 & 0.0264 & II-CT & Sab A & 10.71 & -21.54 & 10.11 & $1.37 \pm 0.03$ & $1.40 \pm 0.03$ \\
\hline UGC 03107 & 0.0273 & II-CT & $\mathrm{Sb} \mathrm{A}$ & 10.65 & -21.54 & 10.90 & $1.45 \pm 0.18$ & $1.43 \pm 0.20$ \\
\hline UGC 03151 & 0.0143 & II.o-CT & $\mathrm{SaAB}$ & 10.59 & -21.62 & 4.68 & $1.39 \pm 0.10$ & $1.35 \pm 0.08$ \\
\hline UGC 03253 & 0.0145 & II.o-OLR & $\mathrm{Sb} \mathrm{B}$ & 10.43 & -21.06 & 5.87 & $1.76 \pm 0.05$ & $1.78 \pm 0.48$ \\
\hline UGC 03539 & 0.0117 & III & $\mathrm{ScAB}$ & 9.83 & -19.67 & 7.41 & $0.94 \pm 0.04$ & $0.85 \pm 0.07$ \\
\hline UGC 03899 & 0.0135 & II-CT & $\mathrm{Sd} A$ & 9.00 & -19.22 & 3.99 & $1.78 \pm 0.05$ & $1.80 \pm 0.05$ \\
\hline UGC 03944 & 0.0136 & II.o-CT & SbcAB & 9.89 & -20.47 & 5.94 & $2.06 \pm 0.04$ & $2.05 \pm 0.04$ \\
\hline UGC 03969 & 0.0275 & II-CT & $\mathrm{Sb} \mathrm{A}$ & 10.68 & -21.16 & 8.69 & $1.62 \pm 0.03$ & $1.64 \pm 0.02$ \\
\hline UGC 03973 & 0.0226 & I & Sbc B & 10.58 & -22.25 & 7.36 & $\ldots$ & $\ldots$ \\
\hline UGC 03995 & 0.0164 & I & $\mathrm{Sb} \mathrm{B}$ & 10.77 & -22.08 & 12.37 & $\ldots$ & $\ldots$ \\
\hline UGC 04029 & 0.0153 & II.o-CT & $\mathrm{ScAB}$ & 10.26 & -20.73 & 8.09 & $2.42 \pm 0.07$ & $2.39 \pm 0.05$ \\
\hline UGC 04054 & 0.0078 & I & Scd A & 9.19 & -19.03 & 3.75 & $\ldots$ & $\ldots$ \\
\hline UGC 04132 & 0.0180 & II.o-CT & SbcAB & 10.71 & -21.78 & 7.69 & $1.32 \pm 0.04$ & $1.32 \pm 0.04$ \\
\hline UGC 04280 & 0.0125 & II.o-CT & $\mathrm{SbAB}$ & 10.09 & -20.32 & 3.56 & $2.19 \pm 0.06$ & $2.21 \pm 0.07$ \\
\hline UGC 04461 & 0.0174 & II-CT & Sbc A & 10.05 & -20.85 & 7.59 & $0.87 \pm 0.03$ & $0.88 \pm 0.02$ \\
\hline UGC 04659 & 0.0070 & II-CT & Sdm A & 8.83 & -18.00 & 3.62 & $1.38 \pm 0.04$ & $1.34 \pm 0.03$ \\
\hline UGC 04722 & 0.0070 & I & Sdm A & 8.71 & -18.20 & 37.47 & $\ldots$ & $\ldots$ \\
\hline UGC 05108 & 0.0280 & II.o-OLR & $\mathrm{Sb} B$ & 10.84 & -22.12 & 16.27 & $1.09 \pm 0.03$ & $1.14 \pm 0.08$ \\
\hline UGC 05111 & 0.0234 & II.o-CT & $\mathrm{SbAB}$ & 10.91 & -21.64 & 8.13 & $1.20 \pm 0.03$ & $1.19 \pm 0.03$ \\
\hline UGC 05244 & 0.0112 & I & Sbc A & 9.29 & -19.18 & 4.82 & $\ldots$ & $\ldots$ \\
\hline UGC 05358 & 0.0109 & II.o-OLR & $\mathrm{Sd} B$ & 9.26 & -19.11 & 4.57 & $1.41 \pm 0.05$ & $1.42 \pm 0.04$ \\
\hline UGC 05359 & 0.0295 & II.o-OLR & $\mathrm{Sb} \mathrm{B}$ & 10.56 & -21.66 & 11.08 & $1.05 \pm 0.06$ & $1.10 \pm 0.04$ \\
\hline UGC 05396 & 0.0194 & II.o-CT & SbcAB & 10.22 & -20.98 & 11.76 & $0.78 \pm 0.04$ & $0.78 \pm 0.04$ \\
\hline UGC 05598 & 0.0202 & II-CT & $\mathrm{Sb} \mathrm{A}$ & 10.19 & -20.77 & 8.22 & $1.65 \pm 0.05$ & $1.68 \pm 0.05$ \\
\hline UGC 05771 & 0.0259 & III-s & E6 A & 11.07 & -22.27 & 8.60 & $1.26 \pm 0.03$ & $1.21 \pm 0.03$ \\
\hline UGC 05990 & 0.0068 & III & Sc A & 9.17 & -18.31 & 1.61 & $0.89 \pm 0.04$ & $0.91 \pm 0.04$ \\
\hline UGC 06036 & 0.0231 & II-CT & $\mathrm{Sa} \mathrm{A}$ & 11.03 & -21.92 & 8.04 & $1.96 \pm 0.06$ & $1.99 \pm 0.07$ \\
\hline UGC 06256 & 0.0126 & II-CT & Sbc A & 9.78 & -18.99 & 5.49 & $1.30 \pm 0.07$ & $1.29 \pm 0.05$ \\
\hline UGC 06312 & 0.0229 & III & Sab A & 10.93 & -21.90 & 12.62 & $0.65 \pm 0.07$ & $0.59 \pm 0.04$ \\
\hline UGC 07012 & 0.0120 & III & ScdAB & 9.11 & -19.86 & 4.41 & $1.21 \pm 0.19$ & $1.20 \pm 0.17$ \\
\hline UGC 07145 & 0.0238 & II-CT & Sbc A & 10.31 & -21.16 & 9.22 & $1.80 \pm 0.03$ & $1.79 \pm 0.04$ \\
\hline UGC 08004 & 0.0225 & II.o-CT & ScdAB & 9.93 & -20.71 & 12.32 & $1.44 \pm 0.04$ & $1.43 \pm 0.03$ \\
\hline UGC 08231 & 0.0099 & II.o-OLR & SdAB & 9.04 & -19.27 & 4.05 & $1.32 \pm 0.05$ & $1.32 \pm 0.03$ \\
\hline UGC 08250 & 0.0196 & II-CT & $\mathrm{Sc} \mathrm{A}$ & 9.79 & -20.12 & 8.08 & $1.23 \pm 0.07$ & $1.22 \pm 0.07$ \\
\hline UGC 08267 & 0.0258 & II.o-CT & SbAB & 10.71 & -21.26 & 8.70 & $1.96 \pm 0.08$ & $1.84 \pm 0.30$ \\
\hline UGC 08322 & 0.0275 & I & SOaAB & 11.05 & -22.24 & 7.21 & $\ldots$ & $\ldots$ \\
\hline UGC 08662 & 0.0087 & II-CT & Scd A & 8.87 & -18.08 & 1.81 & $1.34 \pm 0.04$ & $1.32 \pm 0.03$ \\
\hline UGC 08733 & 0.0097 & I & Sdm B & 9.31 & -19.75 & 4.48 & $\ldots$ & $\ldots$ \\
\hline UGC 08778 & 0.0128 & II-CT & $\mathrm{Sb} \mathrm{A}$ & 10.13 & -20.26 & 2.88 & $1.19 \pm 0.04$ & $1.16 \pm 0.05$ \\
\hline UGC 08781 & 0.0274 & II.o-OLR & $\mathrm{Sb} \mathrm{B}$ & 10.96 & -22.34 & 15.82 & $0.91 \pm 0.06$ & $0.97 \pm 0.05$ \\
\hline
\end{tabular}


Table B.1. continued.

\begin{tabular}{|c|c|c|c|c|c|c|c|c|}
\hline $\begin{array}{l}\text { Name } \\
\text { (1) }\end{array}$ & $\begin{array}{c}z \\
(2)\end{array}$ & $\begin{array}{l}\text { Phot type } \\
\text { (3) }\end{array}$ & $\begin{array}{l}\text { Morph type } \\
\text { (4) }\end{array}$ & $\underset{(5)}{\log \left(M_{*}\right)}$ & $\begin{array}{l}M_{R} \\
(6)\end{array}$ & $\begin{array}{l}R_{\text {eff }} \\
\text { (7) }\end{array}$ & $\begin{array}{l}R_{g^{\prime} \text {,break }} \\
\text { (8) }\end{array}$ & $\begin{array}{l}R_{r^{\prime} \text {,break }} \\
\text { (9) }\end{array}$ \\
\hline UGC 08988 & 0.0086 & II-CT & Scd A & 9.33 & -18.50 & 2.08 & $1.85 \pm 0.14$ & $1.98 \pm 0.22$ \\
\hline UGC 09067 & 0.0283 & III & SbcAB & 10.51 & -21.96 & 8.40 & $1.72 \pm 0.80$ & $3.04 \pm 0.23$ \\
\hline UGC 09071 & 0.0079 & II-CT & Scd A & 8.92 & -18.58 & 4.02 & $0.84 \pm 0.04$ & $0.85 \pm 0.04$ \\
\hline UGC 09080 & 0.0119 & II-CT & $\mathrm{Sc} \mathrm{A}$ & 8.95 & -18.80 & 3.40 & $1.65 \pm 0.11$ & $1.63 \pm 0.08$ \\
\hline UGC 09113 & 0.0126 & III & $\mathrm{SbAB}$ & 9.83 & -19.75 & 5.08 & $1.22 \pm 0.11$ & $1.26 \pm 0.35$ \\
\hline UGC 09165 & 0.0196 & II-CT & $\mathrm{Sa} \mathrm{A}$ & 10.63 & -21.06 & 5.67 & $1.31 \pm 0.05$ & $1.30 \pm 0.05$ \\
\hline UGC 09199 & 0.0280 & I & $\mathrm{SbAB}$ & 10.65 & -21.31 & 4.26 & $\ldots$ & $\ldots$ \\
\hline UGC 09253 & 0.0151 & II.o-CT & ScAB & 9.94 & -20.41 & 4.07 & $2.18 \pm 0.06$ & $2.16 \pm 0.04$ \\
\hline UGC 09291 & 0.0116 & I & Scd A & 9.80 & -20.83 & 5.98 & $\ldots$ & $\ldots$ \\
\hline UGC 09401 & 0.0209 & I & $\mathrm{SaAB}$ & 10.89 & -22.00 & 7.27 & $\ldots$ & $\ldots$ \\
\hline UGC 09448 & 0.0092 & II-CT & $\mathrm{Sb} \mathrm{A}$ & 9.45 & -18.84 & 3.00 & $1.40 \pm 0.09$ & $1.77 \pm 0.16$ \\
\hline UGC 09476 & 0.0127 & II-CT & Sbc A & 10.18 & -20.99 & 4.64 & $1.63 \pm 0.04$ & $1.62 \pm 0.03$ \\
\hline UGC 09492 & 0.0299 & III & SabAB & 11.09 & -22.34 & 9.87 & $1.37 \pm 0.04$ & $1.39 \pm 0.04$ \\
\hline UGC 09537 & 0.0313 & I & $\mathrm{Sb} \mathrm{A}$ & 11.12 & -22.60 & 17.62 & $\ldots$ & $\ldots$ \\
\hline UGC 09542 & 0.0200 & II-CT & $\mathrm{Sc} \mathrm{A}$ & 10.30 & -20.95 & 8.28 & $1.20 \pm 0.15$ & $1.34 \pm 0.14$ \\
\hline UGC 09598 & 0.0204 & II.o-CT & SbcAB & 10.50 & -21.23 & 8.17 & $1.58 \pm 0.02$ & $1.58 \pm 0.02$ \\
\hline UGC 09665 & 0.0103 & II-CT & $\mathrm{Sb} \mathrm{A}$ & 10.04 & -19.99 & 2.81 & $2.21 \pm 0.08$ & $2.28 \pm 0.07$ \\
\hline UGC 09759 & 0.0131 & III & Sab A & 10.14 & -20.21 & 5.51 & $0.94 \pm 0.02$ & $0.94 \pm 0.03$ \\
\hline UGC 09777 & 0.0178 & II-CT & Sbc A & 10.20 & -20.97 & 6.46 & $2.14 \pm 0.11$ & $2.15 \pm 0.05$ \\
\hline UGC 09842 & 0.0315 & I & Sbc B & 10.62 & -21.80 & 12.91 & $\ldots$ & $\ldots$ \\
\hline UGC 09849 & 0.0133 & I & $\mathrm{Sd} A$ & 9.44 & -19.31 & 6.05 & $\ldots$ & $\ldots$ \\
\hline UGC 09873 & 0.0205 & II-CT & $\mathrm{Sb} \mathrm{A}$ & 10.02 & -20.36 & 9.64 & $1.27 \pm 0.14$ & $1.23 \pm 0.12$ \\
\hline UGC 09892 & 0.0207 & II-CT & Sbc A & 10.30 & -20.72 & 9.22 & $1.06 \pm 0.04$ & $1.07 \pm 0.03$ \\
\hline UGC 09901 & 0.0125 & I & $\mathrm{Sc} A$ & 9.37 & -19.37 & 3.58 & $\ldots$ & $\ldots$ \\
\hline UGC 09919 & 0.0126 & II-CT & Sc A & 9.54 & -19.52 & 3.81 & $2.05 \pm 0.06$ & $2.05 \pm 0.06$ \\
\hline UGC 10042 & 0.0162 & II-CT & $\mathrm{Sc} \mathrm{A}$ & 10.31 & -20.68 & 3.80 & $2.52 \pm 0.57$ & $2.65 \pm 0.33$ \\
\hline UGC 10043 & 0.0091 & III & SabAB & 9.80 & -19.30 & 7.79 & $0.26 \pm 0.01$ & $0.66 \pm 0.59$ \\
\hline UGC 10123 & 0.0142 & II-CT & Sab A & 10.51 & -20.55 & 3.84 & $3.19 \pm 1.54$ & $1.21 \pm 1.53$ \\
\hline UGC 10205 & 0.0237 & III-s & SOa A & 11.01 & -22.40 & 8.09 & $2.93 \pm 0.11$ & $3.09 \pm 0.08$ \\
\hline UGC 10257 & 0.0146 & II-CT & Sbc A & 10.00 & -20.44 & 3.36 & $2.27 \pm 0.07$ & $2.18 \pm 0.04$ \\
\hline UGC 10297 & 0.0095 & III & $\mathrm{Sc} \mathrm{A}$ & 9.22 & -19.12 & 4.17 & $1.86 \pm 0.10$ & $2.03 \pm 0.07$ \\
\hline UGC 10337 & 0.0313 & II-CT & $\mathrm{Sb} \mathrm{A}$ & 10.99 & -22.14 & 6.56 & $2.96 \pm 0.07$ & $2.98 \pm 0.05$ \\
\hline UGC 10380 & 0.0311 & III & SbAB & 10.94 & -21.86 & 13.74 & $0.91 \pm 0.05$ & $0.91 \pm 0.05$ \\
\hline UGC 10384 & 0.0186 & III & $\mathrm{Sb} \mathrm{A}$ & 10.31 & -20.73 & 5.07 & $1.60 \pm 0.54$ & $1.60 \pm 0.46$ \\
\hline UGC 10650 & 0.0117 & III & Scd A & 9.32 & -19.37 & 3.36 & $2.97 \pm 0.12$ & $2.05 \pm 0.57$ \\
\hline UGC 10695 & 0.0292 & III-s & E5 A & 10.97 & -22.61 & 13.11 & $1.39 \pm 0.07$ & $1.36 \pm 0.06$ \\
\hline UGC 10710 & 0.0294 & I & $\mathrm{Sb} \mathrm{A}$ & 10.89 & -22.13 & 12.64 & $\ldots$ & $\ldots$ \\
\hline UGC 10796 & 0.0117 & I & ScdAB & 9.28 & -19.49 & 4.75 & $\ldots$ & $\ldots$ \\
\hline UGC 10811 & 0.0303 & II.o-OLR & $\mathrm{Sb} B$ & 10.77 & -21.93 & 12.73 & $1.01 \pm 0.05$ & $1.12 \pm 0.07$ \\
\hline UGC 10905 & 0.0278 & III & S0a A & 11.34 & -22.88 & 11.86 & $1.76 \pm 0.06$ & $1.79 \pm 0.05$ \\
\hline UGC 10972 & 0.0172 & III & Sbc A & 10.33 & -21.20 & 4.93 & $2.95 \pm 0.10$ & $2.95 \pm 0.10$ \\
\hline UGC 11262 & 0.0200 & II-CT & Sc A & 9.87 & -20.21 & 9.93 & $1.25 \pm 0.15$ & $1.17 \pm 0.09$ \\
\hline UGC 11649 & 0.0135 & I & Sab B & 10.39 & -21.29 & 5.56 & $\ldots$ & $\ldots$ \\
\hline UGC 11680NED01 & 0.0264 & III & $\mathrm{Sb} \mathrm{B}$ & 10.70 & -22.48 & 13.05 & $0.98 \pm 0.53$ & $1.46 \pm 0.59$ \\
\hline UGC 11694 & 0.0175 & III-s & SOa A & 11.01 & -22.04 & 9.11 & $2.24 \pm 0.05$ & $2.25 \pm 0.06$ \\
\hline UGC 11717 & 0.0215 & III & Sab A & 10.63 & -21.73 & 29.45 & $0.82 \pm 0.05$ & $0.78 \pm 0.08$ \\
\hline UGC 11740 & 0.0220 & II-CT & Sbc A & 10.26 & -21.25 & 4.96 & $2.28 \pm 0.21$ & $2.14 \pm 0.13$ \\
\hline UGC 11792 & 0.0165 & III & Sbc A & 10.79 & -20.65 & 7.17 & $1.94 \pm 0.13$ & $2.04 \pm 0.07$ \\
\hline UGC 12054 & 0.0073 & II-CT & $\mathrm{Sc} \mathrm{A}$ & 8.95 & -18.34 & 2.41 & $2.02 \pm 0.05$ & $1.89 \pm 0.26$ \\
\hline UGC 12185 & 0.0222 & II.o-OLR & $\mathrm{Sb} \mathrm{B}$ & 10.54 & -21.46 & 7.90 & $1.98 \pm 0.07$ & $1.99 \pm 0.08$ \\
\hline UGC 12224 & 0.0119 & II-CT & $\mathrm{Sc} A$ & 9.92 & -20.80 & 7.28 & $0.88 \pm 0.08$ & $0.92 \pm 0.05$ \\
\hline UGC 12308 & 0.0077 & III & Scd A & 8.96 & -18.87 & 5.41 & $0.84 \pm 0.01$ & $0.84 \pm 0.01$ \\
\hline UGC 12348 & 0.0253 & III & $\mathrm{SbAB}$ & 10.62 & -21.83 & 11.36 & $2.20 \pm 0.06$ & $2.16 \pm 0.05$ \\
\hline UGC 12518 & 0.0094 & II-CT & $\mathrm{Sb} \mathrm{A}$ & 10.11 & -19.41 & 6.18 & $1.37 \pm 0.03$ & $1.41 \pm 0.06$ \\
\hline UGC 12519 & 0.0146 & II.o-CT & $\mathrm{ScAB}$ & 10.07 & -20.55 & 4.57 & $1.92 \pm 0.05$ & $1.95 \pm 0.04$ \\
\hline UGC 12633 & 0.0140 & III & SabAB & 10.19 & -20.93 & 6.59 & $1.11 \pm 0.02$ & $1.11 \pm 0.02$ \\
\hline UGC 12653 & 0.0134 & II-CT & Sc A & 9.79 & -20.24 & 2.52 & $1.89 \pm 0.06$ & $1.90 \pm 0.08$ \\
\hline UGC 12767 & 0.0173 & II.o-OLR & $\mathrm{Sb} \mathrm{B}$ & 10.77 & -22.32 & 11.92 & $1.66 \pm 0.02$ & $1.66 \pm 0.01$ \\
\hline UGC 12810 & 0.0266 & II.o-OLR & Sbc B & 10.67 & -22.01 & 14.89 & $1.73 \pm 0.04$ & $1.71 \pm 0.03$ \\
\hline UGC 12816 & 0.0175 & I & $\mathrm{Sc} \mathrm{A}$ & 9.38 & -20.55 & 8.28 & $\ldots$ & $\ldots$ \\
\hline UGC 12857 & 0.0083 & II-CT & Sbc A & 9.77 & -19.50 & 2.19 & $3.14 \pm 0.08$ & $3.13 \pm 0.07$ \\
\hline UGC 12864 & 0.0155 & III & $\mathrm{Sc} B$ & 9.97 & -20.66 & 7.91 & $2.66 \pm 0.04$ & $2.62 \pm 0.04$ \\
\hline UGC A021 & 0.0064 & I & Sdm B & 9.01 & -19.74 & 4.83 & $\ldots$ & $\ldots$ \\
\hline
\end{tabular}


The following table provides the physical properties derived at break radius for the CALIFA galaxies. The meaning of each column is as follows:

- (1) galaxy name;

- (2) the photometrical type obtained from our analysis of the SDSS $r^{\prime}$-band profiles;

- (3) $R_{r^{\prime} \text {, break }}$, the break radius obtained as the minimum of the double bootstrapping fit to the SDSS $r^{\prime}$-band data in units of their $R_{\text {eff }}$ along with their errors;
- (4) $\Delta \alpha_{(\mathrm{O} / \mathrm{H})}$ represents the difference between the outer-tothe-inner slopes as measured in the oxygen profiles;

- (5) $\Delta \alpha_{\text {color }}$ represents the difference between the outer-to-theinner slopes as measured in the $\left(g^{\prime}-r^{\prime}\right)$ color profiles;

- (6) $\mu_{r^{\prime}, \text { break }}, r^{\prime}$-band surface brightness computed at the break radius in unit of magnitudes/"12;

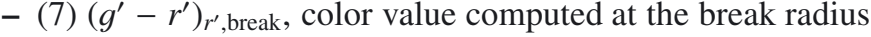
in unit of magnitudes;

- (8) $(\mathrm{O} / \mathrm{H})_{r^{\prime}, \text { break }}$, oxygen abundance value computed at the break radius and expressed in dex. 
A\&A 585, A47 (2016)

Table B.2. Derived physical properties at break radius for the CALIFA galaxies.

\begin{tabular}{|c|c|c|c|c|c|c|c|}
\hline $\begin{array}{l}\text { Name } \\
\text { (1) }\end{array}$ & $\begin{array}{l}\text { PhotT } \\
\text { (2) }\end{array}$ & $\begin{array}{l}\mathrm{R}_{r^{\prime}, \text { break }} \\
\text { (3) }\end{array}$ & $\begin{array}{c}\Delta \alpha_{(\mathrm{O} / \mathrm{H})} \\
(4)\end{array}$ & $\begin{array}{c}\Delta \alpha_{\text {color }} \\
(5)\end{array}$ & $\begin{array}{l}\mu_{r^{\prime}, \text { break }} \\
\text { (6) }\end{array}$ & $\begin{array}{c}\left(g^{\prime}-r^{\prime}\right)_{r^{\prime}, \text { break }} \\
(7)\end{array}$ & $\begin{array}{c}(\mathrm{O} / \mathrm{H})_{r^{\prime}, \text { break }} \\
(8)\end{array}$ \\
\hline 2MASXJ01331766 & II.o-OLR & $1.48 \pm 0.04$ & $0.031 \pm 0.014$ & $0.123 \pm 0.020$ & 21.94 & 0.46 & 8.39 \\
\hline ESO540-G003 & III & $2.12 \pm 0.22$ & $0.025 \pm 0.007$ & $0.104 \pm 0.027$ & 22.28 & 0.46 & 8.51 \\
\hline IC 0995 & II.o-CT & $1.30 \pm 0.04$ & $0.044 \pm 0.024$ & $0.152 \pm 0.038$ & 21.88 & 0.38 & 8.39 \\
\hline IC 1151 & II.o-CT & $1.41 \pm 0.03$ & $0.028 \pm 0.015$ & $0.241 \pm 0.025$ & 22.51 & 0.38 & 8.39 \\
\hline IC 1199 & II.o-OLR & $0.80 \pm 0.04$ & $0.103 \pm 0.092$ & $0.520 \pm 0.093$ & 21.58 & 0.62 & 8.58 \\
\hline IC 1528 & II.o-CT & $2.01 \pm 0.07$ & $0.177 \pm 0.075$ & $0.047 \pm 0.076$ & 23.55 & 0.37 & 8.43 \\
\hline IC 2095 & II.o-CT & $1.18 \pm 0.05$ & $0.124 \pm 0.078$ & $0.096 \pm 0.087$ & 23.12 & 0.40 & 8.15 \\
\hline MCG-02-02-030 & II.o-OLR & $1.19 \pm 0.06$ & $0.014 \pm 0.007$ & $0.135 \pm 0.011$ & 20.91 & 0.66 & 8.57 \\
\hline MCG-02-02-040 & II.o-CT & $2.23 \pm 0.06$ & $0.420 \pm 0.166$ & $0.055 \pm 0.166$ & 22.42 & 0.56 & 8.44 \\
\hline MCG-02-51-004 & II-CT & $1.02 \pm 0.07$ & $0.104 \pm 0.071$ & $0.414 \pm 0.074$ & 21.75 & 0.55 & 8.54 \\
\hline NGC 0001 & III & $2.40 \pm 0.20$ & $0.044 \pm 0.015$ & $0.027 \pm 0.019$ & 23.94 & 0.63 & 8.56 \\
\hline NGC 0023 & III & $1.50 \pm 0.04$ & $0.019 \pm 0.009$ & $0.064 \pm 0.031$ & 22.97 & 0.65 & 8.64 \\
\hline NGC 0036 & II.o-OLR & $1.16 \pm 0.14$ & $0.045 \pm 0.032$ & $0.026 \pm 0.043$ & 22.55 & 0.62 & 8.54 \\
\hline NGC 0171 & II.o-OLR & $1.96 \pm 0.12$ & $0.057 \pm 0.025$ & $0.019 \pm 0.028$ & 23.16 & 0.53 & 8.59 \\
\hline NGC 0177 & II-CT & $1.22 \pm 0.05$ & $0.030 \pm 0.018$ & $0.035 \pm 0.027$ & 21.76 & 0.55 & 8.56 \\
\hline NGC 0180 & II.o-OLR & $1.35 \pm 0.02$ & $0.055 \pm 0.033$ & $0.070 \pm 0.037$ & 22.58 & 0.56 & 8.55 \\
\hline NGC 0192 & II.o-CT & $0.95 \pm 0.02$ & $0.012 \pm 0.007$ & $0.076 \pm 0.016$ & 20.31 & 0.76 & 8.64 \\
\hline NGC 0216 & III & $1.87 \pm 0.08$ & $0.046 \pm 0.017$ & $0.116 \pm 0.019$ & 22.55 & 0.55 & 8.36 \\
\hline NGC 0234 & II.o-CT & $1.38 \pm 0.05$ & $0.019 \pm 0.010$ & $0.141 \pm 0.016$ & 21.85 & 0.63 & 8.56 \\
\hline NGC 0237 & III & $1.63 \pm 0.04$ & $0.043 \pm 0.016$ & $0.205 \pm 0.033$ & 22.39 & 0.46 & 8.52 \\
\hline NGC 0257 & II-CT & $1.21 \pm 0.03$ & $0.036 \pm 0.019$ & $0.255 \pm 0.025$ & 21.45 & 0.56 & 8.57 \\
\hline NGC 0444 & II-CT & $1.53 \pm 0.04$ & $0.073 \pm 0.040$ & $0.012 \pm 0.045$ & 22.80 & 0.51 & 8.43 \\
\hline NGC 0477 & II.o-CT & $1.09 \pm 0.03$ & $0.022 \pm 0.017$ & $0.115 \pm 0.046$ & 22.78 & 0.43 & 8.51 \\
\hline NGC 0551 & II.o-OLR & $1.49 \pm 0.08$ & $0.052 \pm 0.030$ & $0.152 \pm 0.041$ & 21.89 & 0.60 & 8.57 \\
\hline NGC 0681 & III-s & $0.96 \pm 0.12$ & $0.061 \pm 0.049$ & $0.153 \pm 0.049$ & 21.53 & 0.73 & 8.50 \\
\hline NGC 0693 & III & $1.02 \pm 0.04$ & $0.026 \pm 0.019$ & $0.072 \pm 0.023$ & 22.42 & 0.67 & 8.55 \\
\hline NGC 0716 & II.o-CT & $1.56 \pm 0.03$ & $0.038 \pm 0.020$ & $0.151 \pm 0.039$ & 21.56 & 0.56 & 8.57 \\
\hline NGC 0768 & II.o-OLR & $1.74 \pm 0.04$ & $0.119 \pm 0.059$ & $0.018 \pm 0.063$ & 22.90 & 0.40 & 8.49 \\
\hline NGC 0776 & II.o-OLR & $1.18 \pm 0.04$ & $0.027 \pm 0.012$ & $0.319 \pm 0.055$ & 21.71 & 0.60 & 8.60 \\
\hline NGC 0932 & II-CT & $1.11 \pm 0.03$ & $0.031 \pm 0.020$ & $0.219 \pm 0.029$ & 21.90 & 0.85 & 8.55 \\
\hline NGC 1056 & III-s & $0.97 \pm 0.04$ & $0.020 \pm 0.013$ & $0.092 \pm 0.023$ & 22.47 & 0.79 & 8.52 \\
\hline NGC 1093 & III & $1.47 \pm 0.73$ & $0.074 \pm 0.056$ & $0.330 \pm 0.174$ & 23.09 & 0.50 & 8.57 \\
\hline NGC 1677 & III & $1.39 \pm 0.13$ & $0.050 \pm 0.026$ & $0.273 \pm 0.040$ & 22.58 & 0.62 & 8.46 \\
\hline NGC 2730 & II.o-OLR & $1.48 \pm 0.02$ & $0.127 \pm 0.075$ & $0.303 \pm 0.084$ & 23.03 & 0.44 & 8.48 \\
\hline NGC 2805 & III & $0.47 \pm 0.05$ & $0.016 \pm 0.024$ & $0.149 \pm 0.044$ & 22.01 & 0.53 & 8.53 \\
\hline NGC 3381 & III & $1.90 \pm 0.13$ & $0.024 \pm 0.010$ & $0.094 \pm 0.035$ & 22.97 & 0.43 & 8.50 \\
\hline NGC 3614 & II.o-OLR & $0.99 \pm 0.32$ & $0.083 \pm 0.065$ & $0.160 \pm 0.071$ & 22.69 & 0.40 & 8.49 \\
\hline NGC 3687 & II.o-OLR & $1.80 \pm 0.05$ & $0.195 \pm 0.088$ & $0.077 \pm 0.088$ & 23.12 & 0.44 & 8.51 \\
\hline NGC 3811 & III & $1.86 \pm 0.22$ & $0.049 \pm 0.020$ & $0.087 \pm 0.032$ & 23.29 & 0.44 & 8.55 \\
\hline NGC 3994 & III & $1.85 \pm 0.02$ & $0.030 \pm 0.010$ & $0.090 \pm 0.020$ & 23.15 & 0.58 & 8.64 \\
\hline NGC 4149 & II.o-OLR & $2.20 \pm 0.08$ & $0.015 \pm 0.005$ & $0.079 \pm 0.009$ & 20.65 & 0.64 & 8.59 \\
\hline NGC 4210 & II.o-OLR & $1.76 \pm 0.10$ & $0.087 \pm 0.035$ & $0.322 \pm 0.046$ & 22.07 & 0.45 & 8.57 \\
\hline NGC 4711 & II-CT & $1.74 \pm 0.03$ & $0.071 \pm 0.031$ & $0.153 \pm 0.037$ & 21.69 & 0.48 & 8.56 \\
\hline NGC 4961 & II.o-OLR & $1.64 \pm 0.03$ & $0.058 \pm 0.025$ & $0.262 \pm 0.035$ & 22.47 & 0.25 & 8.41 \\
\hline NGC 5000 & III & $1.43 \pm 0.03$ & $0.023 \pm 0.010$ & $0.122 \pm 0.039$ & 22.68 & 0.58 & 8.58 \\
\hline NGC 5267 & II.o-OLR & $1.86 \pm 0.09$ & $0.197 \pm 0.094$ & $0.015 \pm 0.095$ & 22.07 & 0.66 & 8.63 \\
\hline NGC 5289 & II.o-CT & $1.44 \pm 0.02$ & $0.039 \pm 0.020$ & $0.082 \pm 0.022$ & 21.71 & 0.55 & 8.55 \\
\hline NGC 5406 & II.o-OLR & $1.55 \pm 0.05$ & $0.044 \pm 0.018$ & $0.154 \pm 0.023$ & 22.30 & 0.49 & 8.59 \\
\hline NGC 5443 & III & $1.67 \pm 0.04$ & $0.036 \pm 0.018$ & $0.061 \pm 0.020$ & 22.38 & 0.67 & 8.57 \\
\hline NGC 5522 & II.o-OLR & $1.56 \pm 0.02$ & $0.072 \pm 0.040$ & $0.331 \pm 0.042$ & 21.97 & 0.50 & 8.58 \\
\hline NGC 5533 & III & $0.96 \pm 0.06$ & $0.022 \pm 0.017$ & $0.117 \pm 0.036$ & 22.36 & 0.67 & 8.58 \\
\hline NGC 5587 & II-CT & $0.60 \pm 0.02$ & $0.004 \pm 0.003$ & $0.136 \pm 0.010$ & 20.32 & 0.79 & 8.63 \\
\hline NGC 5610 & II.o-OLR & $1.08 \pm 0.03$ & $0.633 \pm 0.521$ & $1.097 \pm 0.535$ & 23.36 & 0.45 & 8.56 \\
\hline NGC 5616 & II.o-CT & $1.20 \pm 0.12$ & $0.046 \pm 0.025$ & $0.099 \pm 0.065$ & 22.05 & 0.56 & 8.58 \\
\hline NGC 5622 & II-CT & $1.53 \pm 0.69$ & $0.077 \pm 0.038$ & $0.132 \pm 0.072$ & 22.65 & 0.44 & 8.51 \\
\hline NGC 5630 & II.o-OLR & $1.16 \pm 0.03$ & $0.046 \pm 0.026$ & $0.122 \pm 0.035$ & 21.48 & 0.27 & 8.35 \\
\hline NGC 5635 & II-CT & $1.20 \pm 0.05$ & $0.025 \pm 0.014$ & $0.151 \pm 0.023$ & 21.51 & 0.71 & 8.57 \\
\hline NGC 5657 & II.o-OLR & $1.82 \pm 0.04$ & $0.078 \pm 0.032$ & $0.105 \pm 0.035$ & 22.98 & 0.46 & 8.47 \\
\hline NGC 5714 & II-CT & $1.28 \pm 0.03$ & $0.027 \pm 0.015$ & $0.129 \pm 0.018$ & 21.59 & 0.56 & 8.52 \\
\hline NGC 5731 & III & $1.40 \pm 0.06$ & $0.026 \pm 0.014$ & $0.184 \pm 0.025$ & 22.34 & 0.29 & 8.36 \\
\hline NGC 5735 & II.o-OLR & $1.74 \pm 0.03$ & $0.106 \pm 0.050$ & $0.115 \pm 0.052$ & 23.14 & 0.45 & 8.52 \\
\hline NGC 5829 & II-CT & $1.88 \pm 0.03$ & $0.097 \pm 0.046$ & $0.315 \pm 0.047$ & 23.58 & 0.35 & 8.42 \\
\hline NGC 5908 & III-s & $2.59 \pm 0.09$ & $0.016 \pm 0.005$ & $0.095 \pm 0.007$ & 22.02 & 0.74 & 8.73 \\
\hline NGC 5947 & II.o-OLR & $1.75 \pm 0.03$ & $0.071 \pm 0.034$ & $0.003 \pm 0.038$ & 22.70 & 0.42 & 8.51 \\
\hline NGC 5980 & II-CT & $1.98 \pm 0.02$ & $0.077 \pm 0.032$ & $0.371 \pm 0.034$ & 21.92 & 0.43 & 8.56 \\
\hline
\end{tabular}


Table B.2. continued.

\begin{tabular}{|c|c|c|c|c|c|c|c|}
\hline $\begin{array}{l}\text { Name } \\
\text { (1) }\end{array}$ & $\begin{array}{l}\text { PhotT } \\
\text { (2) }\end{array}$ & $\begin{array}{l}R_{r^{\prime}, \text { break }} \\
\text { (3) }\end{array}$ & $\begin{array}{c}\Delta \alpha_{(\mathrm{O} / \mathrm{H})} \\
\quad \text { (4) }\end{array}$ & $\begin{array}{l}\Delta \alpha_{\text {color }} \\
\quad(5)\end{array}$ & $\begin{array}{l}\mu_{r^{\prime}, \text { break }} \\
\text { (6) }\end{array}$ & $\begin{array}{c}\left(g^{\prime}-r^{\prime}\right)_{r^{\prime}, \text { break }} \\
(7)\end{array}$ & $\begin{array}{c}(\mathrm{O} / \mathrm{H})_{r^{\prime}} \text {,break } \\
(8)\end{array}$ \\
\hline NGC 6004 & II.o-OLR & $1.50 \pm 0.01$ & $0.026 \pm 0.014$ & $0.198 \pm 0.018$ & 22.10 & 0.61 & 8.58 \\
\hline NGC 6032 & II.o-OLR & $1.69 \pm 0.08$ & $0.024 \pm 0.010$ & $0.026 \pm 0.024$ & 22.70 & 0.65 & 8.54 \\
\hline NGC 6060 & II-CT & $0.87 \pm 0.02$ & $0.039 \pm 0.029$ & $0.201 \pm 0.033$ & 21.22 & 0.68 & 8.57 \\
\hline NGC 6063 & II-CT & $1.20 \pm 0.02$ & $0.027 \pm 0.019$ & $0.119 \pm 0.027$ & 22.08 & 0.48 & 8.51 \\
\hline NGC 6155 & II-CT & $2.15 \pm 0.07$ & $0.037 \pm 0.015$ & $0.176 \pm 0.019$ & 22.64 & 0.54 & 8.55 \\
\hline NGC 6168 & II.o-OLR & $1.89 \pm 0.06$ & $0.038 \pm 0.017$ & $0.059 \pm 0.018$ & 21.73 & 0.60 & 8.40 \\
\hline NGC 6186 & II.o-OLR & $2.19 \pm 0.03$ & $0.041 \pm 0.016$ & $0.186 \pm 0.017$ & 21.48 & 0.64 & 8.63 \\
\hline NGC 6301 & II-CT & $1.21 \pm 0.06$ & $0.022 \pm 0.013$ & $0.037 \pm 0.017$ & 22.02 & 0.53 & 8.54 \\
\hline NGC 6310 & II-CT & $1.21 \pm 0.04$ & $0.010 \pm 0.004$ & $0.024 \pm 0.016$ & 20.94 & 0.73 & 8.59 \\
\hline NGC 6478 & II-CT & $1.17 \pm 0.06$ & $0.035 \pm 0.016$ & $0.168 \pm 0.042$ & 20.94 & 0.55 & 8.57 \\
\hline NGC 6497 & II.o-OLR & $0.74 \pm 0.01$ & $0.151 \pm 0.178$ & $0.441 \pm 0.181$ & 22.47 & 0.61 & 8.61 \\
\hline NGC 7321 & II.o-OLR & $1.60 \pm 0.07$ & $0.032 \pm 0.014$ & $0.094 \pm 0.017$ & 22.49 & 0.50 & 8.55 \\
\hline NGC 7364 & III & $1.40 \pm 0.35$ & $0.015 \pm 0.007$ & $0.027 \pm 0.013$ & 22.07 & 0.70 & 8.60 \\
\hline NGC 7489 & II-CT & $1.77 \pm 0.03$ & $0.126 \pm 0.067$ & $0.539 \pm 0.067$ & 23.04 & 0.46 & 8.32 \\
\hline NGC 7549 & III & $1.25 \pm 0.04$ & $0.033 \pm 0.021$ & $0.070 \pm 0.036$ & 22.52 & 0.71 & 8.56 \\
\hline NGC 7591 & II.o-OLR & $1.40 \pm 0.05$ & $0.047 \pm 0.026$ & $0.235 \pm 0.039$ & 22.31 & 0.66 & 8.60 \\
\hline NGC 7691 & II.o-CT & $1.51 \pm 0.03$ & $0.167 \pm 0.099$ & $0.183 \pm 0.101$ & 23.34 & 0.48 & 8.41 \\
\hline NGC 7716 & III & $1.18 \pm 0.33$ & $0.089 \pm 0.043$ & $0.091 \pm 0.124$ & 21.68 & 0.49 & 8.55 \\
\hline NGC 7782 & II-CT & $0.99 \pm 0.03$ & $0.009 \pm 0.005$ & $0.196 \pm 0.020$ & 21.32 & 0.69 & 8.58 \\
\hline NGC 7800 & III & $1.59 \pm 0.17$ & $0.058 \pm 0.025$ & $0.067 \pm 0.065$ & 22.58 & 0.38 & 8.34 \\
\hline NGC 7819 & II-CT & $1.43 \pm 0.05$ & $0.033 \pm 0.016$ & $0.222 \pm 0.019$ & 22.86 & 0.42 & 8.49 \\
\hline SDSSJ100141 & II.o-CT & $1.68 \pm 0.04$ & $0.266 \pm 0.138$ & $0.059 \pm 0.139$ & 24.11 & 0.18 & 8.11 \\
\hline UGC 00139 & III & $1.39 \pm 0.06$ & $0.035 \pm 0.018$ & $0.198 \pm 0.042$ & 23.05 & 0.33 & 8.42 \\
\hline UGC 00148 & II-CT & $1.53 \pm 0.07$ & $0.017 \pm 0.006$ & $0.120 \pm 0.011$ & 21.37 & 0.51 & 8.44 \\
\hline UGC 00312 & II.o-OLR & $1.79 \pm 0.13$ & $0.116 \pm 0.054$ & $0.134 \pm 0.061$ & 23.26 & 0.30 & 8.34 \\
\hline UGC 00809 & II-CT & $1.69 \pm 0.03$ & $0.125 \pm 0.057$ & $0.266 \pm 0.058$ & 22.32 & 0.53 & 8.35 \\
\hline UGC 00841 & II-CT & $1.26 \pm 0.03$ & $0.069 \pm 0.038$ & $0.032 \pm 0.040$ & 22.09 & 0.56 & 8.48 \\
\hline UGC 00987 & III & $1.14 \pm 0.04$ & $0.014 \pm 0.007$ & $0.087 \pm 0.014$ & 21.86 & 0.71 & 8.63 \\
\hline UGC 01057 & II.o-OLR & $1.73 \pm 0.06$ & $0.078 \pm 0.034$ & $0.668 \pm 0.044$ & 23.26 & 0.34 & 8.41 \\
\hline UGC 01659 & II.o-OLR & $1.31 \pm 0.03$ & $0.125 \pm 0.071$ & $0.010 \pm 0.079$ & 22.44 & 0.63 & 8.50 \\
\hline UGC 01749 & II.o-OLR & $1.01 \pm 0.01$ & $0.194 \pm 0.157$ & $0.063 \pm 0.158$ & 22.23 & 0.79 & 8.57 \\
\hline UGC 02403 & III & $2.21 \pm 0.04$ & $0.039 \pm 0.013$ & $0.106 \pm 0.024$ & 23.00 & 0.73 & 8.64 \\
\hline UGC 02405 & II-CT & $1.07 \pm 0.05$ & $0.047 \pm 0.028$ & $0.344 \pm 0.082$ & 21.88 & 0.50 & 8.52 \\
\hline UGC 03253 & II.o-OLR & $1.76 \pm 0.05$ & $0.127 \pm 0.061$ & $0.171 \pm 0.062$ & 23.16 & 0.54 & 8.54 \\
\hline UGC 03539 & III & $0.94 \pm 0.04$ & $0.085 \pm 0.068$ & $0.440 \pm 0.071$ & 22.50 & 0.42 & 8.48 \\
\hline UGC 03899 & II-CT & $1.78 \pm 0.05$ & $0.092 \pm 0.045$ & $0.259 \pm 0.049$ & 23.27 & 0.27 & 8.23 \\
\hline UGC 04132 & II.o-CT & $1.32 \pm 0.04$ & $0.028 \pm 0.014$ & $0.134 \pm 0.022$ & 21.41 & 0.67 & 8.57 \\
\hline UGC 05108 & II.o-OLR & $1.09 \pm 0.03$ & $0.167 \pm 0.119$ & $0.267 \pm 0.122$ & 23.08 & 0.53 & 8.59 \\
\hline UGC 05111 & II.o-CT & $1.20 \pm 0.03$ & $0.030 \pm 0.016$ & $0.058 \pm 0.028$ & 21.32 & 0.87 & 8.56 \\
\hline UGC 05358 & II.o-OLR & $1.41 \pm 0.05$ & $0.057 \pm 0.030$ & $0.176 \pm 0.037$ & 23.50 & 0.29 & 8.35 \\
\hline UGC 05359 & II.o-OLR & $1.05 \pm 0.06$ & $0.040 \pm 0.023$ & $0.120 \pm 0.031$ & 22.08 & 0.54 & 8.54 \\
\hline UGC 05396 & II.o-CT & $0.78 \pm 0.04$ & $0.039 \pm 0.028$ & $0.345 \pm 0.041$ & 21.72 & 0.49 & 8.50 \\
\hline UGC 07012 & III & $1.21 \pm 0.19$ & $0.019 \pm 0.010$ & $0.024 \pm 0.047$ & 23.25 & 0.29 & 8.40 \\
\hline UGC 07145 & II-CT & $1.80 \pm 0.03$ & $0.136 \pm 0.065$ & $0.203 \pm 0.066$ & 23.16 & 0.46 & 8.46 \\
\hline UGC 08231 & II.o-OLR & $1.32 \pm 0.05$ & $0.076 \pm 0.043$ & $0.446 \pm 0.046$ & 22.52 & 0.14 & 8.25 \\
\hline UGC 08250 & II-CT & $1.23 \pm 0.07$ & $0.258 \pm 0.157$ & $0.168 \pm 0.157$ & 22.40 & 0.47 & 8.38 \\
\hline UGC 08781 & II.o-OLR & $0.91 \pm 0.06$ & $0.144 \pm 0.101$ & $0.152 \pm 0.105$ & 22.39 & 0.47 & 8.59 \\
\hline UGC 09067 & III & $1.72 \pm 0.80$ & $0.115 \pm 0.031$ & $0.215 \pm 0.083$ & 22.93 & 0.40 & 8.46 \\
\hline UGC 09071 & II-CT & $0.84 \pm 0.04$ & $0.023 \pm 0.018$ & $0.066 \pm 0.049$ & 22.15 & 0.43 & 8.38 \\
\hline UGC 09598 & II.o-CT & $1.58 \pm 0.02$ & $0.093 \pm 0.046$ & $0.149 \pm 0.048$ & 22.47 & 0.53 & 8.50 \\
\hline UGC 09665 & II-CT & $2.21 \pm 0.08$ & $0.130 \pm 0.046$ & $0.249 \pm 0.047$ & 22.41 & 0.37 & 8.59 \\
\hline UGC 09759 & III & $0.94 \pm 0.02$ & $0.022 \pm 0.015$ & $0.122 \pm 0.029$ & 22.30 & 0.64 & 8.49 \\
\hline UGC 09777 & II-CT & $2.14 \pm 0.11$ & $0.610 \pm 0.251$ & $0.133 \pm 0.252$ & 24.59 & 0.26 & 8.49 \\
\hline UGC 09873 & II-CT & $1.27 \pm 0.14$ & $0.251 \pm 0.170$ & $0.164 \pm 0.172$ & 22.55 & 0.55 & 8.48 \\
\hline UGC 09892 & II-CT & $1.06 \pm 0.04$ & $0.041 \pm 0.029$ & $0.267 \pm 0.031$ & 22.17 & 0.53 & 8.51 \\
\hline UGC 10257 & II-CT & $2.27 \pm 0.07$ & $0.033 \pm 0.013$ & $0.065 \pm 0.017$ & 22.01 & 0.47 & 8.43 \\
\hline UGC 10384 & III & $1.60 \pm 0.54$ & $0.110 \pm 0.045$ & $0.231 \pm 0.078$ & 22.19 & 0.44 & 8.48 \\
\hline UGC 10811 & II.o-OLR & $1.01 \pm 0.05$ & $0.046 \pm 0.028$ & $0.045 \pm 0.069$ & 21.94 & 0.61 & 8.58 \\
\hline UGC 11262 & II-CT & $1.25 \pm 0.15$ & $0.170 \pm 0.120$ & $0.071 \pm 0.123$ & 23.59 & 0.43 & 8.44 \\
\hline UGC 11740 & II-CT & $2.28 \pm 0.21$ & $0.076 \pm 0.020$ & $0.155 \pm 0.021$ & 22.41 & 0.55 & 8.51 \\
\hline UGC 12224 & II-CT & $0.88 \pm 0.08$ & $0.033 \pm 0.024$ & $0.090 \pm 0.144$ & 22.23 & 0.52 & 8.52 \\
\hline UGC 12308 & III & $0.84 \pm 0.01$ & $0.027 \pm 0.021$ & $0.025 \pm 0.066$ & 22.39 & 0.38 & 8.32 \\
\hline UGC 12519 & II.o-CT & $1.92 \pm 0.05$ & $0.132 \pm 0.056$ & $0.298 \pm 0.057$ & 21.60 & 0.51 & 8.48 \\
\hline UGC 12767 & II.o-OLR & $1.66 \pm 0.02$ & $0.124 \pm 0.065$ & $0.290 \pm 0.066$ & 23.43 & 0.66 & 8.58 \\
\hline UGC 12810 & II.o-OLR & $1.73 \pm 0.04$ & $0.305 \pm 0.155$ & $0.103 \pm 0.158$ & 23.66 & 0.45 & 8.51 \\
\hline UGC 12864 & III & $2.66 \pm 0.04$ & $0.135 \pm 0.045$ & $0.088 \pm 0.048$ & 25.11 & 0.36 & 8.41 \\
\hline
\end{tabular}

